Der Medizinischen Fakultät der Georg-August-Universität Göttingen eingereicht von Prof. Dr. med. Uwe Fischer

\title{
Zuverlässigkeit bildgebender Verfahren in der Früherkennung des Mammakarzinoms
}

\author{
INAUGURAL-DISSERTATION \\ zur Erlangung des Doktorgrades \\ der Medizinischen Fakultät \\ der Georg-August-Universität zu Göttingen
}

vorgelegt von

Isabel Kampmann-Küster

aus

Göttingen

Göttingen 2012 
Dekan: Prof. Dr. med. M. P. Schön

I. Berichterstatter: Prof. Dr. med. U. Fischer

II. Berichterstatter/in: Prof. Dr. med. J. Meller

III. Berichterstatter/in: Prof. Dr. med., Dr. rer. nat. T.A. Crozier

Tag der mündlichen Prüfung: 29.08.2012 


\section{Inhaltsverzeichnis}

$\begin{array}{ll}\text { Einleitung } & 7\end{array}$

1. Biologie und Klassifikation des Mammakarzinoms 8

1.1 Tumorentstehung $\quad 8$

1.2 Grundlagen der Tumorangiogenese $\quad 8$

1.3 Klassifikation maligner Tumoren der Mamma 9

1.3.1 Intraduktales Mammakarzinom (duktales Carcinoma in situ, DCIS, duktale intraepitheliale Neoplasie) $\quad 9$

1.3.2 Invasive Mammakarzinome 12

1.3.3 Sonderformen des Mammakarzinoms 14

1.4 Prognosefaktoren des Mammakarzinoms $\quad 15$

1.4.1 Etablierte Prognosefaktoren 16

$\begin{array}{ll}\text { 1.4.2 Neue Prognosefaktoren } & 20\end{array}$

2. Früherkennung des Mammakarzinoms 21

2.1 Früherkennung $\quad 21$

$\begin{array}{ll}2.2 \text { Anamnese } & 21\end{array}$

2.3 Eigenuntersuchung und klinische Untersuchung 22

2.4 Röntgen-Mammographie 22

2.5 Mammasonographie $\quad 24$

$\begin{array}{ll}\text { 2.6 Mamma-MRT } & 26\end{array}$

3. Material und Methoden $\quad 28$

$\begin{array}{ll}3.1 \text { Kollektiv } & 28\end{array}$

$\begin{array}{ll}3.2 \text { Untersuchungsverfahren } & 29\end{array}$

$\begin{array}{ll}3.3 \text { Befundauswertung } & 29\end{array}$ 
$\begin{array}{ll}4.1 \text { Kollektiv } & 35\end{array}$

$\begin{array}{ll}\text { 4.2 Ergebnisse Mammographie } & 37\end{array}$

4.3 Ergebnisse Ultraschall 43

4.4 Ergebnisse Mamma-MRT $\quad 46$

4.5 Perkutane Biopsie $\quad 51$

4.6 Ergebnisse aller bildgebenden Verfahren 52

5. Diskussion 61

$\begin{array}{ll}5.1 \text { Brustkrebs im frühen Stadium } & 61\end{array}$

5.2 Therapeutisches Vorgehen bei präinvasiven bzw. früh-invasiven Tumoren 61

5.3 Überleben und Langzeitüberleben $\quad 64$

5.4 Frühdiagnostik des Mammakarzinoms 65

5.5.1 Stellenwert der Mammographie in der Früherkennung 66

5.5.2 Stellenwert der Mammasonographie in der Früherkennung 68

5.5.3 Stellenwert der Mamma-MRT in der Früherkennung 70

5.5.4 Stellenwert von Mammographie und Sonographie in der Früherkennung 72

5.5.5 Stellenwert von Mamma-MRT und Sonographie in der Früherkennung 73

5.5.6 Stellenwert von Mammographie und Mamma-MRT in der Früherkennung 74

5.5.7 Stellenwert von Mammographie, Sonographie und Mamma-MRT in der

$\begin{array}{ll}\text { Früherkennung } & 75\end{array}$

$\begin{array}{ll}\text { 5.5.8 Perspektiven } & 77\end{array}$ 
7. Literatur

$\begin{array}{lr}\text { 8. Anhang } & 94\end{array}$

$\begin{array}{ll}\text { 8.1 Abbildungsverzeichnis } & 94\end{array}$

8.2 Tabellenverzeichnis 96

$\begin{array}{ll}\text { 8.3 Abkürzungsverzeichnis } & 97\end{array}$ 


\section{Einleitung}

Der Nachweis des Mammakarzinoms in einem frühen Stadium ist eine wesentliche Voraussetzung für eine gute Prognose dieser Erkrankung. Unter Früherkennung wird im Allgemeinen die Detektion des Tumors im intraduktalen Stadium (DCIS) verstanden oder aber - bei invasiven Karzinomen - eine Tumorgröße unter $10 \mathrm{~mm}$. Für diese frühen Stadien kann davon ausgegangen werden, dass das Langzeitüberleben in einer Größenordnung von 95\% liegt, sofern eine adäquate Therapie der Tumoren erfolgt.

Die regelmäßige Selbstuntersuchung und die klinische Untersuchung durch Ärzte sind für die Früherkennung von Brustkrebs bekanntermaßen nicht geeignet. Im Gegensatz hierzu erlauben bildgebende Untersuchungverfahren eine deutlich frühere Diagnose des Mammakarzinoms. Etablierte Untersuchungsmethoden stellen in diesem Zusammenhang die Röntgenmammographie, die Mammasonographie und die kontrastmittelgestützte Mamma-MRT dar. Hierbei basieren alle drei Verfahren auf einem unterschiedlichen Ansatz zur Diskriminierung bösartiger Tumoren gegenüber dem gesunden Umgebungsgewebe. Die Mammographie nutzt das unterschiedliche Absorptionsverhalten verschiedener Gewebearten gegenüber niederenergetischen Röntgenstrahlen aus. Die Sonographie visualisiert das Reflexions- und Absorptionsmuster von Ultraschallwellen. Bei der MRT wird das Durchblutungsverhalten verschiedener Gewebearten im zeitlichen Verlauf durch die periphervenöse Applikation eines Blutfarbstoffes sichtbar gemacht.

In der vorliegenden Studie wurde geprüft, wie hoch die Zuverlässigkeit jedes dieser drei Untersuchungsverfahren für die Früherkennung des Mammakarzinoms ist. Hierbei wurde zwischen den Tumorstadien pTis, pT1a und pT1b unterschieden, wobei in der Gruppe der intraduktalen Karzinome zwischen dem low-grade-, dem intermediate und dem high-grade-Typ differenziert wurde. Zudem wurde hinterfragt, in welchem Zusammenhang der positive Nachweis eines früh erkannten Mammakarzinoms mit dem mammographischen, sonographischen oder magnetresonanztomographischen Grundmuster des Drüsenparenchyms steht.

Die Zielsetzung der Studie war es, den Stellenwert der drei bildgebenden Verfahren (Mammographie, Ultraschall, Mamma-MRT) für die prognostisch wichtige Früherkennung von Brustkrebs auf der Basis eines einheitlich untersuchten Kollektives zu bestimmen und in diesem Kontext zu evaluieren, welche Kriterien für das jeweilige Untersuchungsverfahren führend waren. 


\section{Biologie und Klassifikation des Mammakarzinoms}

\subsection{Tumorentstehung}

Tumoren entwickeln sich durch eine Veränderung der sonst exakt regulierten Entwicklung und Vermehrung von Zellen. Diese Veränderung kann durch chemische, physikalische und virale Noxen, sowie durch erbliche, endokrine und ernährungsbedingte Faktoren oder das Lebensalter ausgelöst werden. In diesem Prozess spielen die unkontrollierte Aktivierung von Onkogenen und der Verlust bzw. die Inaktivierung von Tumorsuppressorgenen eine wichtige Rolle. Folglich kommt es zur Zellkommunikationsstörung mit Aufhebung der Kontaktinhibition, Proliferationsenthemmung, Differenzierungsstörungen und immunologischen Störungen [Riede und Schäfer 1999].

\subsection{Grundlagen der Tumorangiogenese}

Die nutritive Versorgung maligner Tumoren der Mamma, die primär fast ausnahmslos innerhalb des Milchgangssystems ihren Ursprung haben, erfolgt in der Anfangsphase der Tumorentstehung über Diffusion. Mit zunehmendem Größenwachstum sind solide Tumoren abhängig von einem mitwachsenden Kapillarnetz, das den Tumor mit Sauerstoff und Metaboliten versorgt (sog. tumorinduzierte Angiogenese oder Tumorangiogenese). Anhand von Tierversuchen konnte eindrucksvoll gezeigt werden, dass maligne Tumoren bis zu einer Größenordnung von etwa 1-2 mm über Diffusion ernährt werden, da die entsprechende Schichtdicke offensichtlich für das diffusionsgestützte Feeding reicht. Ab $2 \mathrm{~mm}$ erlaubt erst die Ausbildung eines suffizienten Kapillarnetzes das Weiterwachsen des Tumors, da die Diffusionsstrecke in dieser Phase des Tumorwachstums zur ausreichenden Versorgung des Karzinoms nicht mehr ausreicht [Folkman und Klagsbrun 1987; Pluda 1997].

Bei der Neubildung tumorassoziierter Gefäße handelt es sich um einen komplexen Prozess, bei dem die zur Bildung der Gefäßwände notwendigen Endothelzellen, die sich in einem Monate oder Jahre dauernden Ruhezustand befinden können, durch verschiedene angiogenetische Wachstumsfaktoren, etwa basic Fibroblast Growth Factor (bFGF) und Vascular Endothelial Growth Factor (VEGF) aktiviert werden. Zahlreiche weitere Faktoren sind inzwischen bekannt [Fischer 2010].

Im Rahmen der Gefäßneubildung wird das kapillarumgebende Bindegewebe lysiert und es erfolgt eine Zellmigration kleiner Zellausläufer in das Gewebe. Neue Kapillaren entstehen durch Proliferation und Migration von vorherbestehenden Endothelzellen. Lose Kapillaren besitzen eine erhöhte Permeabilität mit konsekutivem Austritt von Plasmaeiweißen. 
Die spätere Umwandlung der Kapillaren in Arteriolen, Arterien, Venolen und Venen stellt den Abschluss des durch Angiogenesefaktoren getriggerten Prozesses der Angiogenese dar und wird durch die Aktivierung bestimmter Botenstoffe festgelegt [Folkman und Klagsbrun 1987].

\subsection{Klassifikation maligner Tumoren der Mamma}

Die malignen Tumoren der Mamma werden grundsätzlich in zwei Gruppen unterteilt: Zum einen in die Gruppe der nicht-invasiven, intraduktalen Karzinome, die noch nicht lymphogen oder hämatogen gestreut haben. Zum anderen in die Gruppe der invasiven Karzinome, die das Milchgangssystem verlassen haben, potentiell das Risiko einer Metastasierung mit sich bringen und daher als systemische Erkrankung des gesamten Körpers verstanden werden. Nachfolgend werden die verschiedenen Malignome der Mamma erläutert, wobei insbesondere auf Epidemiologie, histologische Besonderheiten und typische Befundkonstellationen in klinischer und bildgebender Diagnostik eingegangen wird.

\subsubsection{Intraduktales Mammakarzinom (duktales Carcinoma in situ, DCIS, duktale intraepitheliale Neoplasie)}

Das duktale Carcinoma in situ (DCIS) stellt ein biologisch und morphologisch heterogenes Krankheitsbild dar. Es ist charakterisiert durch eine Verbreitung von epithelialen malignen Zellen im Lumen der Milchgänge ohne Nachweis einer Invasion über die Basalmembran hinaus [Goussia et al. 2006]. Es ist meist unizentrisch und segmental beschränkt [Dronkers et al. 1999].

Das DCIS kann fünf verschiedene Wachstumsmuster aufweisen: Komedo-, solider, kribriformer, mikropapillärer und papillärer Typ. Beim Auftreten mehrerer Komponenten spricht man von einem Mischtyp. Komedonekrosen sind Ausdruck einer nutritiv-defizitären und hypoxischen Zellschädigung. Die verschiedenen Wachstumsmuster des DCIS unterscheiden sich auch hinsichtlich des DNA-Gehalts, der Proliferationsaktivität, Hormonrezeptorausstattung und Potenz zur Stromainvasion [Mammakarzinom: Manual Diagnostik und Therapie 2005].

Die Pathogenese der DCIS-Entwicklung ist derzeit noch nicht geklärt. Die kontinuierliche Entwicklung von der Hyperplasie zum Karzinom ist umstritten. Aufgrund des Wachstumsmusters und durch immunhistochemische Reaktionen lassen sich duktale Hyperplasien von neoplastischen Proliferaten eines DCIS in der Regel unterscheiden [Mammakarzinom: Manual Diagnostik und Therapie 2005].

Im Jahre 1995 stellten Silverstein et al. eine neue prognostische Klassifikation des DCIS, die Van-Nuys-Klassifikation vor. Die Van-Nuys-Klassifikation stützt sich lediglich auf die beiden morphologischen Kriterien Kerngrading und Komedonekrosen. Für die Therapie stellte sich das Kerngrading als wichtigster 
prognostischer Parameter für das lokale Rezidiv heraus. Die Van-Nuys-Klassifikation definiert drei DCIS-Gruppen anhand des Kerngradings und des Vorhandenseins von Komedo-Nekrosen. Die aggressivste Gruppe (high-grade, Gruppe 3) wird unabhängig von möglichen Nekrosen allein durch ein hohes Kerngrading festgelegt (Kerngrad 3) [Silverstein et al. 1995].

Die DCIS-Fälle mit niedrigem Kerngrading (non-high-grade) werden durch die Anoder Abwesenheit von Komedo-Nekrosen in die Gruppe 1 (ohne Nekrosen, lowgrade) und Gruppe 2 (mit Nekrosen, intermediate) unterteilt [Tumorzentrum Hamburg E.V.http://www.tumorzentrumhh.de/Leitlinien/dcis.html].

\begin{tabular}{lllll}
\hline Score & $\mathbf{1}$ & $\mathbf{2}$ & 3 \\
\hline Grad & Niedriger & Grad, keine & Niedriger & Grad \\
& Nekrose & Nekrose & Hoher Grad \\
Größe $(\mathrm{mm})$ & $1-15$ & $16-40$ & \\
Rand $(\mathrm{mm})$ & $>10$ & $1-9$ & $>40$ \\
Alter $($ Jahre $)$ & $>60$ & $40-60$ & $<1$ \\
\hline
\end{tabular}

Tab.1.3.1a: Van-Nuys-Prognose-Index für DCIS [Silverstein und Buchanan 2003]

Gruppe 1: Prognose-Index 4-6, geringes Risiko

Gruppe 2: Prognose-Index 7-9, mittleres Risiko

Gruppe 3: Prognose-Index 10-12, hohes Risiko

Das schlecht differenzierte DCIS zeigt pleomorphe und irregulär geformte Nuklei mit verklumptem Chromatin und erhöhter Mitoseaktivität. Die architektonische Differenzierung ist aufgehoben oder nur minimal vorhanden. Das Wachstumsmuster ist solide, pseudo-kribriform oder mikropapillär (ohne zelluläre Polarisation). Nekrosen sind in der Regel nachweisbar. Verkalkungen, falls vorhanden, stellen sich amorph dar [Holland et al. 1994]. Das intermediär differenzierte DCIS besteht aus Zellen, die einige Pleomorphismen aufweisen, jedoch nicht so wie in der schlecht differenzierten Gruppe. Hier zeigt sich eine Polarisation im Interzellularraum. Variationen in Form, Größe und Oberfläche sind eher gering. Die Mitoseaktivität ist ebenfalls gering. Darüber hinaus werden Nekrosen und Kalzifikationen insgesamt seltener beobachtet als in der Gruppe der schlecht differenzierten DCIS [Holland et al. 1994].

Beim gut differenzierten DCIS finden sich Zellen, die monomorphe, regelmäßig angeordnete kleine Nuklei mit feinem Chromatin und unauffälligen Nukleoli besitzen. Die Zellen zeigen eine ausgeprägte apikale Polarisierung. Das Wachstumsmuster stellt sich sowohl kribriform, mikropapillär oder solide dar. Nekrosen sind beim gut differenzierten DCIS ungewöhnlich. Verkalkungen sind teilweise vorhanden [Holland et al. 1994]. 


\section{Diagnostischer Nachweis des DCIS (Mammographie, Ultraschall und MR- Mammographie)}

Das DCIS ist in der Regel klinisch stumm, nur selten zeigen sich tumorassoziierte Veränderungen, wie z.B. eine pathologische Mamillensekretion oder ein palpabler Tumor. Ca. $65 \%$ der duktalen Karzinome in situ weisen suspekte Mikrokalzifikationen auf [Kreienberg et al. 2006].

Die Mammographie detektiert in-situ-Karzinome hauptsächlich durch den Nachweis von tumorassoziierten Mikroverkalkungen. DCIS kann mammographisch allerdings auch als Herdbefund oder Architekturstörung imponieren. Ca. 35\% der DCIS sind mammographisch okkult [Heywang-Köbrunner und Schreer 2003]. Bei den schlecht differenzierten DCIS wird häufig in den intraduktalen Tumorsträngen eine Nekrose gefunden, die meist amorphe Verkalkungen enthält. Im Mammogramm erscheinen diese Kalzifikationen linear, verzweigend oder grobgranulär. Bei den intermediär differenzierten DCIS erscheinen die Verkalkungen mammographisch grobgranulär, feingranulär, aber auch linear. Bei den gut differenzierten DCIS zeigen sich mammographisch gruppierte feingranuläre Verkalkungen [Holland et al. 1994; Dronkers et al. 1999].

Sonographisch ist das DCIS aufgrund der nicht ausreichenden räumlichen Auflösung und der reduzierten Beurteilung der Milchgangslumina nur selten nachweisbar. In einer Untersuchung von Park et al. konnte gezeigt werden, dass bei sonographischer Detektion eines high-grade-DCIS Mikrokalzifikationen mit assoziierter Gangveränderung im Vordergrund standen, bei den non-high-grade-DCIS ein irregulärer mikrolobulierter Randsaum [Park et al. 2010]. Nach Literaturangaben sind aber nur ca. $20 \%$ der mammographisch sichtbaren Mikrokalzifikationen auch sonographisch darstellbar [Soo et al. 2002].

Auch die Mamma-MRT ist nicht in der Lage, die feinen karzinomassoziierten Verkalkungen in der Brust direkt abzubilden. Das DCIS wird überwiegend per diffusionem ernährt und erst in der Phase der Invasion der Basalmembran erfolgt die Tumorneoangiogenese, die daraus resultierende Vaskularisation wird dann bildlich dargestellt. Dies erklärt die geringe Nachweisrate von 50 bis $70 \%$ in der MRMammographie [Westerhof et al. 1996]. In Analogie zum Verteilungsmuster intraduktaler Mikroverkalkungen zeigt auch die MR-Mammographie beim DCIS typischerweise nicht raumfordernde Veränderungen (sog. Non-mass-like-lesions) mit linearer, dendritischer oder segmentaler Aufnahme des Kontrastmittels. Die Detektion des DCIS in der MRT erfolgt in erster Linie aufgrund von morphologischen Kriterien, während die Signalkurvenanalyse keine Rolle spielt [Fischer und Baum 2009]. Mit der Entwicklung offener Spulen und der damit verbundenen Reduktion der Bewegunsartefakte sowie dem Einsatz hochauflösender Untersuchungsprotokolle (Matrix 512x512) wurden die Ergebnisse der MRT für den Nachweis von DCIS zunehmend besser. Studien der letzten Jahre belegen inzwischen, dass die MammaMRT für den Nachweis von DCIS eine höhere Sensitivität aufweist als die Mammographie [Berg et al. 2004; Kuhl et al. 2010]. 


\subsubsection{Invasive Mammakarzinome}

Nachfolgend werden die verschiedenen Tumorentitäten, die zu den invasiven Mammakarzinomen zählen, präsentiert.

\section{Invasiv duktales Mammakarzinom (ID)}

Das invasiv duktale Mammakarzinom (ID) ist mit 40 bis $75 \%$ der häufigste Tumortyp. Er umfasst eine heterogene Gruppe von Tumoren, die nicht in ausreichendem Umfang Charakteristika aufweisen, um einem speziellen histologischen Typ zugeordnet werden zu können. Das makromorphologische Wachstumsmuster ist variabel und reicht von knotigen bis hin zu diffus infiltrierenden Erscheinungsformen.

Bedingt durch das typischerweise langsame Tumorwachstum und das Vorherrschen von Tumorstroma kommt es im charakteristischen Fall zur Ausbildung eines Szirrhus mit strahlenförmiger Begrenzung des Tumors.

Um als invasiver duktaler Typ klassifiziert zu werden, muss ein Tumor ein nicht spezialisiertes Muster in über $50 \%$ seiner Matrix zeigen [S3-Leitlinie ... Mammakarzinom 2008].

In Abhängigkeit von der Größe und Lage eines invasiv duktalen Mammakarzinoms sind die Tumoren klinisch okkult oder als Knoten bzw. Verhärtung palpabel. Sie können zu Kutis- oder Mamillenretraktion führen. Mammographisch zeigt sich das invasiv duktale Mammakarzinom als Herd mit zentraler Dichtesteigerung, Größendiskrepanz in Bezug auf den Tastbefund und variabler Konfiguration. Tumorassoziierte Mikroverkalkungen finden sich in etwa 30\% der Fälle [Rosen und Oberman 1993]. Sonographisch erkennt man unscharf begrenzte, echoarme bis echoleere Herdbefunde, die häufig einen echoreichen Randsaum aufweisen. In der MR-Mammographie findet sich oft ein pathologisches Signalverhalten in der KManreichernden Läsion mit einem starken initialen Enhancement und einer postinitialen Plateauphase oder einem „wash-out"-Phänomen [Fischer 2010].

\section{Invasiv duktales Karzinom mit extensiver intraduktaler Komponente (ID+EIC)}

Ist das invasiv duktale Karzinom mit einer extensiven intraduktalen Tumorkomponente assoziiert, bei dem ein DCIS-Anteil von mindestens $25 \%$ über die Grenzen des invasiven Tumoranteils hinausreicht, so wird von einer begleitenden EIC gesprochen [Rosen und Oberman 1993]. Für das invasive duktale Karzinom mit EIC wurde ein erhöhtes Lokalrezidivrisiko beschrieben [Gage et al. 1996; Frykberg und Bland 1994]. 


\section{Invasiv lobuläres Karzinom (IL)}

Die invasiv lobulären Karzinome (IL) machen 10 bis $14 \%$ aller Mammakarzinome aus. Es wird durch kleine Tumorzellen, starke Zelldissoziation und Bildung von Zellreihen in einem stark fibrosierten Stroma charakterisiert [Bubendorf et al. 2011].

Die Diagnose des IL ist gelegentlich erschwert, da diese Tumorform nur selten mit Zystenformationen, Nekrosen, Hämorrhagien oder Kalzifikationen vergesellschaftet ist. In Abhängigkeit von der Tumorgröße erscheint das IL klinisch stumm oder als Verhärtung. Mammographisch imponiert es meist als schwer zu definierende Verdichtung, parenchymale Asymmetrie oder Architekturstörung [Rodenko et al. 1996]. Sonographisch zeigt es keine spezifischen Charakteristika oder morphologische Veränderungen, die denen in der Mammographie ähneln. In der MRT zeigt es sich oft als „Non-mass-like-lesion“.

Die Signalcharakteristik ist häufig malignomtypisch mit steilem initialen Anstieg und anschließendem Auswascheffekt [Dietzel et al. 2011]. Allerdings kann das lobuläre Mammakarzinom mit unspezifischen Signalkurven einhergehen, so dass für diese Tumorform eher morphologische Kriterien bei der Diagnosefindung im Vordergrund stehen [Fischer 2010].

\section{Medulläres, muzinöses und tubuläres Mammakarzinom}

Das medulläre Karzinom fällt als gut abgegrenzter markiger Knoten auf. Histologisch ist der Tumor sehr zellreich und stromaarm. Er besteht aus großen und differenzierten Zellen, welche dichte Zellnester bilden. Das spärliche Stroma ist unterschiedlich stark durch Lymphozyten und Plasmazellen infiltriert. Dies schließt auf eine gute Immunabwehr, denn der Tumor hat trotz Mitosereichtum und geringer Gewebsdifferenzierung eine bessere Prognose als die invasiven duktalen Karzinome [Riede und Schäfer 1999]. Es macht insgesamt weniger als 7\% aller invasiven Mammakarzinome aus [Samir et al. 2011]. In einer Untersuchung von Yilmaz et al. wurden typische und atypische medulläre Karzinome sonographisch und mammographisch untersucht. Hierbei zeigte sich bei beiden Tumorarten sowohl mammographisch als auch sonographisch eine scharfe Tumorbegrenzung. Ein für Karzinome typischer posteriorer Schallschatten konnte sonographisch allerdings nicht gefunden werden [Yilmaz et al. 2002].

In der Mamma-MRT ist das medulläre Karzinom charakterisiert durch stark vaskulierte rundliche Herdsetzungen mit mikrolobulierter Begrenzung und eher hohem Signal in der T-2-Gewichtung, was für einen hohen Wassergehalt der Tumormatrix spricht [Fischer 2010].

Das tubuläre Mammakarzinom ist durch seine hohe Gewebsreife klassifiziert und besteht aus Gangwucherungen in tubulären Formationen. Diese Tubuli werden durch eine einzige Reihe uniformer Epithelien austapeziert. Sie sind reichlich von sklerosierendem Stroma umgeben und wachsen ins Mammafettgewebe (G1-Tumor) 
[Riede und Schäfer 1999]. Tubuläre Karzinome werden in der Regel mammographisch als irregulär geformte Masse mit zentraler Verdichtung und spikulierten Rändern erkannt, sonographisch als echoarme Struktur mit undefiniertem Randsaum und dorsalem Schallschatten. Allerdings ist das tubuläre Karzinom schwer von einer radiären Narbe zu unterscheiden [Sheppard et al. 2000].

Sonographisch und mammographisch zeigt sich diese Tumorart unspezifisch. Die Mamma-MRT zeigt typischerweise eine spikulierte Läsion mit gesteigerter Vaskularisation. Das T-2-Signal ist eher gering. Die Signal-Zeit-Kurven sind häufig unspezifisch [Fischer 2010].

Das muzinöse Karzinom wächst in Form eines gut abgegrenzten Knotens mit gallertiger Schnittfläche. Dies ist darauf zurückzuführen, dass die Tumorepithelien exzessive Schleimmengen produzieren und sie in Form von Schleimseen größtenteils im Extrazellularraum deponieren. Die uniformen Tumorzellen bilden solide oder drüsenartige Zellzapfen und enthalten manchmal auch in ihrem Zytoplasma noch Schleim [Riede und Schäfer 1999]. Die häufigste mammographische Erscheinung der muzinösen Karzinome ist eine definierte Masse mit klar definierten Randstrukturen, sonographisch zeigt sich eine echoarme Läsion mit innerem heterogenem Echo [Liu et al. 2010].

Es fällt im MRT typischerweise durch einen hohen Wassergehalt auf, der sich durch ein hohes Signal im T-2-Bild erkennen lässt. Das Enhancement nach KM-Gabe ist oft karzinomtypisch [Fischer 2010].

Für die Gruppe der tubulären, der medullären und der muzinösen Karzinome ist eine günstige Prognose bekannt [Garne et al. 1994].

\subsubsection{Sonderformen des Mammakarzinoms}

M. Paget und inflammatorische Formen des Mammakarzinoms werden zu den Sonderformen gezählt.

\section{Morbus Paget}

Makroskopisch imponiert eine ekzematöse, teilweise mit Krustenbildung und Ulzerationen einhergehende Veränderung im Bereich der Mamille und Areola. Hierbei handelt es sich um die Manifestation eines distalen Milchgangskarzinoms [Bastert 1990]. Histologisch fallen in der akanthotisch verdickten Epidermis einzelne oder gruppenförmig zusammengelagerte große sogenannte Paget-Zellen mit auffällig hellem Zytoplasma auf [Riede und Schäfer 1999].

Klinisch äußert sich diese Veränderung als Ekzem oder ulzerierende Hautveränderung im Bereich der Mamille. Mammographisch ist manchmal eine Abflachung oder eine Verdickung der Mamillenregion zu erkennen. Zudem liegen in 
etwa $30 \%$ der Fälle retromamilläre Mikrokalzifikationen vor [Rosen und Oberman 1993]. Sonographisch lassen sich häufig keine charakteristischen Befunde erheben. In der MRT zeigt sich eine unspezifische Signalanreicherung. Die Prognose wird durch das assoziierte Karzinom sowie durch mögliche weitere intramammäre Tumormanifestationen definiert.

\section{Inflammatorisches Mammakarzinom}

Das inflammatorische Mammakarzinom beschreibt keinen bestimmten histologischen Karzinomtyp, sondern eine spezielle Ausbreitungsart eines invasiven Karzinoms. Es handelt sich in der Regel um eine ausgedehnte Lymphangiosis carcinomatosa der kutanen und subkutanen Lymphgefäße. Dieser Lymphstau verursacht ein kutanes und interstitielles Ödem [Bubendorf et al. 2011].

Klinisch imponiert die Trias Schwellung, Rötung und Schmerz. Mammographisch können herdförmige Verdichtungen, aber auch Mikroverkalkungen vorkommen [Heywang-Köbrunner und Scheer 2003]. Die differentialdiagnostische Abgrenzung einer Mastitis nonpuerperalis von einem inflammatorischen Mammakarzinom ist schwierig [Barth und Prechtel 1990], da die sonographischen Kriterien einer Mastitis nonpuerperalis nahezu identisch mit denen eines inflammatorischen Karzinoms sind. Die Kriterien können unter anderem ein interstitielles und subkutanes Ödem mit echoleeren Spalten und verdickter Haut sein [Sohn et al. 2003].

In einer Untersuchung von Le-Petross et al. zeigten sich in der MR-Mammographie viele kleine konfluierende heterogene Anreicherungen und eine generelle Hautverdickung [Le-Petross et al. 2011]. Trotzdem erlaubt auch die Mamma-MRT keine zuverlässige Differenzierung zwischen einem inflammatorischen Mammakarzinom und einem entzündlichen Geschehen. Die Diagnosestellung erfolgt histopathologisch [Fischer 2010]. Die Prognose ist üblicherweise schlecht.

\subsection{Prognosefaktoren des Mammakarzinoms}

Der Prognosefaktor ist ein biologischer, histopathologischer, biochemischer oder klinischer Parameter, der den Verlauf von Mammakarzinomen beeinflusst [Kubli et al. 1984]. Zur optimierten individuellen Therapie im Sinne des chirurgischen Vorgehens oder einer adjuvanten Radio- bzw. Chemotherapie sind diese Parameter essentiell. Unterschieden werden etablierte Prognoseparameter und neue Prognoseparameter. 


\subsubsection{Etablierte Prognosefaktoren}

Nachfolgend werden die etablierten Prognosefaktoren und ihre Bedeutung erläutert.

\section{TNM-Status}

Die TNM-Klassifikation gibt Auskunft über die klinische (cT) und pathologische Größe des Tumors (pT), den Lymphknotenbefall (N) und eine potentielle Fernmetastasierung (M). Sie wurde im Jahr 2002 entsprechend den wissenschaftlichen Fortschritten neu überarbeitet [Singletary et al. 2002]. Sie ist unverzichtbar für das therapeutische Vorgehen und für die prognostische Einschätzung eines Mammakarzinoms [Knörr et al. 1989].

\section{Größe des Primärtumors (T-Stadium)}

Von entscheidender Bedeutung für die Prognose des Mammakarzinoms ist die Größe des Primärtumors. Mit zunehmender Tumorgröße steigt die Wahrscheinlichkeit eines Befalls axillärer Lymphknoten, das Risiko für ein Rezidiv und das Auftreten von Fernmetastasen [Atkinson et al. 1986; Arriagada et al. 1992]. Insgesamt sinkt damit die Überlebenswahrscheinlichkeit [Young et al. 1984].

\section{Lymphknotenbefall (N-Stadium)}

Der axilläre Nodalstatus ist der prognostisch wichtigste Parameter für den Verlauf des Mammakarzinoms [Carter et al.1989; Bonadonna et al. 1995; Oven Ustaalioglu et al. 2010]. Die lokoregionäre Ausbreitung erfolgt vor allem in die axillären Lymphknoten, aber auch in die supraklavikulären und retrosternalen Lymphknoten [Kett et al. 2002; Wulf und Schmidt-Matthiesen 1996].

So wird bei Betrachtung der Anzahl befallener Lymphknoten über 5-JahresÜberlebenszeiten von $96 \%$ bei Nodal-negativem Status, von $79 \%$ bei ein bis drei befallenen Lymphknoten und von $70 \%$ bei vier oder mehr befallenen Lymphknoten berichtet [Sutherland und Mather 1986]. Der axilläre Lymphknotenstatus repräsentiert die lokale Streuung, so dass sich die Tendenz zur Fernmetastasierung voraussagen lässt [Carter et al. 1989]. Auch in der adjuvanten Therapieentscheidung spielt der axilläre Lymphknotenstatus eine wichtige Rolle. Oft ist er ein wesentlicher Faktor für die Entscheidungsfindung für oder gegen eine Chemotherapie [Levine et al.1998]. Seit der Konsensus-Konferenz von St. Gallen 2003 werden Nodal-positive Patientinnen als "high risk" eingestuft [Kaufmann et al. 2004].

Der Lymphknotenstatus ist nicht alleine Ausdruck der Chronologie des Tumorwachstums, sondern zeigt auch die biologische Aggressivität des Tumors 
[Kreienberg et al. 2006]. Die Klassifikation des lokoregionären Lymphknotenbefalls erfolgt in den Stufen NO-N3.

\section{Fernmetastasierung (M-Stadium)}

Die hämatogene Metastasierung findet bevorzugt in das Skelettsystem statt $(70 \%)$ [Arnerlöv et al. 1990]. Desweiteren erfolgt die Metastasierung in Lunge (60\%), Leber (50\%) und Gehirn (15-30\%) [Riede und Schäfer 1993]. Die Unterteilung einer potentiellen Fernmetastasierung erfolgt in den Kategorien M0 und M1.

\section{Histopathologisches Grading}

Bloom und Richardson entwickelten ein „zelluläres Grading“, das eine Einteilung in gut, mäßig und schlecht differenzierte Karzinome erlaubt. Berücksichtigt werden hierbei die tubulären Formationen, der Kernpolymorphismus und die Mitoserate mittels eines Punktesystems. Die unterschiedlichen Malignitätsgrade des Gradings korrelieren mit der Überlebenszeit [Bloom und Richardson 1957]. Es besteht eine Beziehung zwischen der Tumorzell-Differenzierung und dem rezidivfreien Überleben. Hochdifferenzierte Karzinome (G1) besitzen eine Gesamtüberlebensrate von etwa $75 \%$, mäßig gut differenzierte Karzinome (G2) von etwa $50 \%$ und schlecht differenzierte Karzinome (G3) eine von etwa 30\% [Bässler 1984]. Das von Bässler et al. überarbeitete Grading-System ist in Tabelle1.4.1a aufgeführt.

\begin{tabular}{|c|c|}
\hline Kriterien & Score \\
\hline \multicolumn{2}{|l|}{ Tubulusausbildung } \\
\hline$>75 \%$ & 1 \\
\hline $10-75 \%$ & 2 \\
\hline$<10 \%$ & 3 \\
\hline \multicolumn{2}{|l|}{ Kernpolymorphismus } \\
\hline gering & 1 \\
\hline mittelgradig & 2 \\
\hline stark & 3 \\
\hline \multicolumn{2}{|l|}{ Mitoserate } \\
\hline 0-+/HPF3(0-10/10 HPF) & 1 \\
\hline 1-2/HPF (11-20/10 HPF) & 2 \\
\hline$>2 / \mathrm{HPF}(>20 / 10 \mathrm{HPF})$ & 3 \\
\hline
\end{tabular}

Tab.1.4.1a: Grading-System [Bässler et al. 1992]

Summenscore 3-5, Grading G1: gut differenziert, gering maligne

Summenscore 6-7, Grading G2: mäßig differenziert, mäßig maligne

Summenscore 8-9, Grading G3: schlecht differenziert, hoch maligne

HPF: high power field (größtmögliche mikroskopische Auflösung) 


\section{Alter}

Die Mammakarzinominzidenz steigt mit zunehmendem Alter bis zur Menopause rapide an. Danach lässt sich weiterhin eine Zunahme der Brustkrebserkrankungen verzeichnen, jedoch mit einem weniger steilen Verlauf der Inzidenzkurve [Colditz 1993].

In einer Studie von Botteri et al. konnte gezeigt werden, dass das Alter eng mit Tumorgröße, Tumorfortschritt und positivem Nodalstatus (ca. ab 65 Jahren) korreliert. Der Grund hierfür liegt aber in der Tatsache, dass kein systematisches Screeningprogramm für diese Subpopulation existiert und ältere Patientinnen den Arzt später konsultieren als jüngere Patientinnen. Das Alter erhöht per se nicht das Risiko für die Entstehung von Lymphknotenmetastasen [Botteri et al. 2010].

Ein niedriges Erkrankungsalter ist ein ungünstiger Prognosefaktor für das Mammakarzinom [Borger et al. 1994]. Bonnier et al. wiesen in einer Studie nach, dass Frauen unter 35 Jahren häufiger Lokalrezidive, größere Tumoren und mehr G3Karzinome aufwiesen und damit eine signifikant schlechtere Überlebensrate hatten [Bonnier et al. 1995]. Diese Patientengruppe profitiert jedoch auch am meisten von einer adjuvanten Chemotherapie [Kroman et al. 2000].

Ob das Alter einen prognostischen Faktor darstellt, wird in diversen Studien kontrovers diskutiert. Jayasinghe et al. konnten zeigen, dass die Verbindung von Alter mit Tumorgröße, Lymphknotenbefall und histopathologischem Grading einen unabhängigen Faktor für das Gesamtüberleben darstellt. Es sollte lediglich für die therapeutische Entscheidungsfindung in Betracht gezogen werden [Jayasinghe et al. 2005].

\section{Hormonrezeptorstatus}

In etwa zwei Dritteln aller Mammakarzinome können spezifische Rezeptoren für Östrogen und Progesteron nachgewiesen werden [Clark et al. 1984]. Östrogene aus der Zirkulation und andere Steroidhormone können in die Zelle eindringen und am Östrogenrezeptor binden. Dieser Komplex stimuliert die nukleare DNA zur Synthese von Proteinen und zu Wachstum und Proliferation der Zelle [Donegan 1992]. Beim Vorliegen eines positiven Östrogen-Rezeptorstatus ist auch eine verlängerte Überlebenszeit zu verzeichnen, sowohl bei Lymphknoten- positiven, als auch bei Lymphknoten-negativen Tumoren [Vollenweider-Zerargui et al. 1986]. Eine Verbesserung der Prognose ist festzustellen, wenn sowohl ein positiver Östrogenrezeptorstatus als auch ein positiver Progesteronrezeptorstatus vorliegen [Fisher B et al. 1988]. Der Östrogenrezeptor-Status ist nur in den ersten postoperativen Jahren ein günstiger Prognosefaktor. Im Verlauf verliert er seine Aussagekraft bei der Beurteilung des Langzeitüberlebens. Der Nachweis von Progesteronrezeptoren geht wesentlich enger mit einer günstigen Prognose einher und ist ein zusätzlich prädiktiver Faktor für die Wirksamkeit einer hormonellen 
Therapie [Bardou et al. 2003]. Nach der Definition der AGO (Arbeitsgemeinschaft für gynäkologische Onkologie) besteht eine antihormonelle Behandlungsindikation, wenn mehr als $10 \%$ der Tumorzellen den Östrogen- und/oder den Progesteronrezeptor exprimieren. Der Hormonrezeptor besitzt sowohl prognostische als auch prädiktive Bedeutung [Arbeitsgemeinschaft für gynäkologische Onkologie E.V. 2011].

\section{HER2/neu-Status}

Das HER2/neu-Onkoprotein ist ein transmembranöser Wachstumsfaktorrezeptor vom Tyrosinkinase-Typ und ca. $20 \%$ bis $25 \%$ der invasiven Mammakarzinome zeigen eine Überexpression dieses Glykoproteins [Chang 2010]. Es korreliert oft mit einem rasch progredienten, häufig metastasierenden klinischen Verlauf und ist assoziiert mit anderen ungünstigen Prognosefaktoren. Therapeutisch werden anthrazyklinhaltige, insbesondere taxanhaltige Chemotherapien angewendet [Mammakarzinom: Manual Diagnostik und Therapie 2005]. Es gibt Mitteilungen, dass eine Assoziation mit einer Chemotherapieresistenz und möglicherweise auch einer endokrinen Therapieresistenz besteht [Konecny et al. 2000].

Bei gleichzeitigem positiven Östrogen-Rezeptorstatus und HER2-Positivität scheinen Hormonentzugsbehandlungen (GnRH-Analoga, Aromatasehemmer) im Gegensatz zu Tamoxifen günstigere Behandlungseffekte zu ermöglichen [Shou et al. 2004].

Die HER2/neu-Überexpression ist ein prädiktiver Faktor für das Ansprechen auf eine Therapie mit dem spezifischen Antikörper Trastuzumab (Herceptin). Den internationalen Empfehlungen folgend, sollte die Indikation zur Herceptintherapie auf starker immunhistochemischer Expression von HER2/neu $(=3+)$ beruhen. Bei mittelgradiger Expression $(=2+)$ muss die ergänzende $\mathrm{FISH}$-Untersuchung erfolgen. Hierbei sind etwa 25\% dieser Patientinnen FISH-positiv und nur bei diesen sollte Herceptin eingesetzt werden [Kreienberg et al. 2006].

\section{Gefäßinvasion}

Invasion in den Tumor umgebende Blut- oder Lymphgefäße geht mit einem erhöhten Rezidivrisiko einher [Kato et al. 2003]. In einer multivarianten Analyse konnte die Lymphgefäßinvasion als unabhängiger Faktor für das krankheitsfreie Überleben und für das Gesamtüberleben ermittelt werden [Schoppmann et al. 2004]. Seit der Konsensus-Konferenz 2005 in St. Gallen wird die Gefäßinvasion als etablierter negativer prognostischer Faktor angesehen und geht in die Risikoklassifikation bei Nodal-negativen Patientinnen ein [Mammakarzinom: Manual Diagnostik und Therapie 2005]. 


\subsubsection{Neue Prognosefaktoren}

Neben den klassischen Prognosefaktoren werden die "neuen“ Prognosefaktoren wie z.B. p53, EGFR, uPA/PAI-1, S-Phase-Fraktion, Ki-67 und Mitoseindex als potentiell prognostisch und/oder prädiktiv diskutiert. Die klinische Bedeutung bzw. der Nutzen wird jedoch kritisch betrachtet. 


\section{Früherkennung des Mammakarzinoms}

\subsection{Früherkennung}

Die Prognose des Mammakarzinoms hängt neben einer Vielzahl anderer Faktoren entscheidend von der Tumorgröße und insbesondere vom Befall der lokoregionären Lymphknoten ab. Die Zielsetzung in der Diagnostik des Mammakarzinoms ist es daher, diese Erkrankung in einem möglichst frühen Stadium zu erkennen. Unter Früherkennung von Brustkrebs wird die Entdeckung als präinvasive Form oder als frühes invasives Stadium, in dem die 5-Jahres-Überlebensrate bei adäquater Therapie über $90 \%$ liegt verstanden [S3-Leitlinie... Mammakarzinom 2008]. Dies bedeutet typischerweise eine Größe invasiver Karzinome von unter $10 \mathrm{~mm}$.

Zur Früherkennung von Mammakarzinomen kommen verschiedene Untersuchungsverfahren zum Einsatz. Neben der Anamneseerhebung, der Eigenuntersuchung und der klinischen Untersuchung kommt insbesondere den bildgebenden Verfahren eine übergeordnete Bedeutung zu. Zu den etablierten Untersuchungsverfahren zählen die Mammographie, die Mammasonographie und die Mamma-MRT.

Die Farbdopplersonographie ohne bzw. mit Einsatz von Echosignalverstärkern, die Infrarot-Thermographie, die Elastographie, die 99m-Technetium-MIBI-Szintigraphie, die Tomosynthese, die kontrastmittelverstärkte Spektralmammographie und die Positronen-Emmissions-Tomographie spielen in der Frühdiagnostik des Mammakarzinoms keine oder nur eine sehr untergeordnete Rolle.

\subsection{Anamnese}

Die Anamneseerhebung dient der Erfassung von Risikofaktoren, die in allgemeine und determinierende Risikofaktoren unterteilt werden. $\mathrm{Zu}$ den allgemeinen Risikofaktoren gehören u.a. Geschlecht, Alter, Zeitpunkt der Menarche sowie Menopause und Höhe des Body-Mass-Index (BMI). Zusätzlich können ein hohes Alter bei Erstgeburt, Nulliparität sowie Alkohol- und Nikotinkonsum risikoerhöhend wirken [Förster et al. 1998; S3-Leitlinie... Mammakarzinom 2008]. Zu den determinierenden Risikofaktoren gehören u.a die genetische Prädisposition, insbesondere in Form einer Mutation des BRCA-1 oder BRCA-2-Gens, die Strahlenexposition und Hormonersatztherapie mit kombinierten Östrogenen und Gestagenen [Mammakarzinom: Manual Diagnostik und Therapie 2005]. 


\subsection{Eigenuntersuchung und klinische Untersuchung}

Die Eigenuntersuchung bzw. klinische Untersuchung umfasst zunächst die Inspektion beider Mammae hinsichtlich Formveränderung, Mamillenretraktion und Hautbeschaffenheit. Die klinische Untersuchung beinhaltet die Palpation der Brust, das Auffinden von Resistenzen, die gezielte Untersuchung der Mamillen und Perimamillärregion und das Abtasten der Lymphknotenstationen axillär sowie infraund supraklavikulär.

Die Selbstuntersuchung der Brust trägt wesentlich zur individuellen Motivation und Bewusstseins-Förderung für präventive Maßnahmen (sog. awareness) bei [Mammakarzinom: Manual Diagnostik und Therapie 2005]. Sie ist insbesondere bei Frauen unter 40 Jahren wichtig, da für diese Altersgruppe bei Fehlen einer Hochrisikokonstellation keine Indikation zur Mammographie besteht [Howard und Scott-Findlay 2006]. Allerdings stellt die Selbstuntersuchung keine hinreichend effiziente Form der Früherkennung dar, da bösartige Tumoren durchschnittlich erst in einer Größenordnung von 2-3 cm getastet werden [Mammakarzinom: Manual Diagnostik und Therapie 2005].

\subsection{Röntgen-Mammographie}

Bei der Röntgen-Mammographie erfolgt eine Untersuchung des Brustdrüsengewebes unter Verwendung niederenergetischer (low energy) Röntgenstrahlung. Das Absorptionsverhalten der verschiedenen Gewebekomponenten erlaubt hierbei ein Abbild der intramammären Strukturen in Form eines Röntgenmammogramms. Die Durchführung der Röntgenmammographie erfolgte über viele Jahre in konventioneller Film-Folien-Technik und gegenwärtig in zunehmendem Maße in digitaler Technik, wobei digitale Aufnahmen primär als Lumineszenzradiographie, zunehmend jedoch als Vollfeldmammographie erfolgen. Die digitale Röntgenmammographie besitzt gegenüber der konventionellen Technik mehrere Vorteile: Zum einen resultiert eine bis 25\%ige Dosisreduktion, zum anderen findet sich eine kontinuierlich gute Bildqualität. In Studien wurden Vorteile der digitalen Technik insbesondere bei dichten Mammae und bei prämenopausalen Frauen gefunden [Pisano et al. 2005].

Die Untersuchung erfolgt üblicherweise als sogenannte 2-Ebenen-Mammographie. Hierbei stellen die Untersuchung im medio-lateral schrägen Strahlengang (MLO) und im kranio-kaudalen Strahlengang (CC) die zwei Standardebenen dar. Die Kriterien einer guten Einstellungstechnik und Bildqualität werden über das PGMI-System (PGMI= Perfect, Good, Moderate, Inadequate), das dem britischen MammographieScreening entstammt, erfasst. Für bestimmte Fragestellungen können zusätzliche Spezialaufnahmen angefertigt werden. Hierzu zählen insbesondere die Anfertigung einer dritten Aufnahmeebene (medio-lateral (ML), latero-medial (LM)), Vergrößerungsaufnahmen (magnification view) zur besseren physikalischen 
Auflösung von Mikroverkalkungen und die Tubuskompressionsaufnahme (spot view) zur besseren Darstellung potentiell überlagerter Gewebeabschnitte. Weitere Spezialaufnahmen betreffen die Kleopatra-Aufnahme zur Darstellung weit lateral gelegener Prozesse, gedrehte oder überkippte Aufnahmen und die sogenannte Busen-oder Cleavage-Aufnahme für weit medial gelegene Prozesse [Roth-Ganter 2002].

Die Auswertung der Mammographie erfolgt in Anlehnung an das American College of Radiology. Erfasst werden der Dichtetyp (Typ I-IV nach ACR), der Typus auffälliger Befunde (Herd, Verkalkungen und Architekturstörung) sowie die Wahrscheinlichkeit für das Vorliegen eines malignen Prozesses (BI-RADS-Kategorisierung in Stufen 0$6)$.

Bei Herdbefunden handelt es sich - im Gegensatz zu Verdichtungen - um raumfordernde Veränderungen, die gewöhnlich in zwei orthogonalen Ebenen sichtbar sind. Prinzipiell erfolgt die Deskription eines Herdbefundes anhand der Form (rund, oval, lobulär, irregulär), der Begrenzung (scharf abgrenzbar, mikrolobuliert, überlagert, unscharf, spikuliert) und der Dichte (hyperdens, isodens, hypodens, fettäquivalent) [American College of Radiology (ACR) 2003].

Bei der Analyse intramammärer Verkalkungen wird primär unterschieden zwischen eindeutig gutartigen Kalzifikationen und solchen, die auf Malignität verdächtig sind. Gutartige Verkalkungen sind in der Regel größer als Kalzifikationen maligner Genese. Sie sind gröber, oft rundlich, glatt begrenzt und einfach zu detektieren. Typisch gutartig sind Hautverkalkungen, vaskuläre Verkalkung mit parallelen Bahnen oder linear tubuläre Verkalkungen, die eindeutig Blutgefäßen zuzuordnen sind, grobe, korzenzieherartige oder popcorn-ähnliche Verkalkungen, große astartige Verkalkungen, rundliche Verkalkungen, Verkalkungen vom Eierschalen- bzw. RingTyp und Kalkmilch-Verkalkungen (Teetassen-Verkalkungen). Verkalkungen, die mit Malignität assoziiert sind, sind in typischer Weise klein (Mikroverkalkungen). Suspekte Verkalkungen zeigen sich mammographisch als amorphe oder unscharfe Kalzifikate, granuläre heterogene oder pleomorphe Verkalkungen sowie fein-lineare Mikrokalzifikationen. Verkalkungen zeigen verschiedene Verteilungsmuster, die von diffus oder regional über gruppiert, linear und segmental unterschieden werden [American College of Radiology (ACR) 2003].

Bei den Architekturstörungen ist die normale Architektur des Parenchyms radiär gestört, wobei kein definitiver Herdbefund sichtbar ist. Sie umfassen Spikulierungen, die radiär von einem Punkt ausgehen und fokale Retraktionen oder Gefügestörungen im Drüsenparenchym darstellen. Architekturstörungen können mit Herdbefunden, Asymmetrie oder Verkalkungen assoziiert sein. Liegen keine Hinweise auf ein vorausgegangenes Trauma oder eine Operation vor, so ist eine Architekturstörung verdächtig auf eine radiäre Narbe oder einen bösartigen Mammaprozess [American College of Radiology (ACR) 2003]. 
Die Tomosynthese ist eine neue Technologie der digitalen Mammographie. Diese ermöglicht eine Verbesserung der Sichtbarkeit von Brustläsionen durch die Kombination von Tomographie und 3-D-Technik [Baldwin 2009]. Die Voraussetzung ist eine bewegliche Röntgenröhre am Mammographiegerät, mittels derer die Brust aus verschiedenen Winkeln aufgenommen werden kann. In einer Untersuchung von Kopans et al. konnte gezeigt werden, dass durch die Tomosynthese im Vergleich zur konventionellen Mammographie Mikrokalzifikationen gleich oder besser dargestellt werden konnten [Kopans et al. 2011]. Somit könnte dieses Verfahren in der Früherkennung eine zunehmende Rolle spielen.

\subsection{Mammasonographie}

Die Sonographie (B-Bild) ist eine wichtige Ergänzungsmethode zur Mammographie. Sie sollte zudem zur Abklärung klinisch nicht tastbarer, auffälliger oder unklarer mammographischer Befunde eingesetzt werden. Indiziert ist der Einsatz dieser Methode bei klinischen Auffälligkeiten, unklaren Veränderungen im Mammogramm sowie bei dichten und damit mammographisch nur begrenzt beurteilbaren Brüsten [Berg und Gilbreath 2000]. Bei symptomatischen Befunden soll bei Frauen jünger als 40 Jahre die Sonographie primär als bildgebende Methode der Wahl eingesetzt werden. Als alleinige Methode zur Früherkennung von Brustkrebs wird die Mammasonographie als nicht geeignet angesehen [Lorenz et al. 2001].

Für die Mammasonographie kommen Scanner mit einer Frequenz von $5 \mathrm{MHz}$ bis 13 $\mathrm{MHz}$ zur Anwendung. Der auf eine Gewebsschicht auftreffende Schallimpuls kann grundsätzlich eine Reflexion, Dämpfung oder Absorption erfahren. Diese Phänomene resultieren aus den unterschiedlichen Leitfähigkeiten der verschiedenen Gewebekomponenten und dem Echoverhalten an den Trennflächen unterschiedlich dichter Gewebestrukturen [Madjar 2010].

Das Ziel einer standardisierten durchgeführten Mammasonographie ist die systematische und reproduzierbare Durchuntersuchung beider Mammae und der Axillae [Schulz-Wendtland et al. 2003]. Die Mammasonographie dient der Differenzierung zwischen soliden und zystischen Prozessen und der weiteren Tumorspezifizierung [Boetes et al. 1995].

Die Domäne der Mammasonographie ist die Detektion und Charakterisierung von Herdbefunden, die hauptsächlich hinsichtlich ihrer Form (oval, rund, irregulär), ihrer Orientierung (parallel oder antiparallel, größter vertikaler Durchmesser), ihrer Begrenzung (umschrieben, unscharf, Konturwinkelungen, Mikrolobulierungen, Spikulierungen), der Echogenität (anechoisch, hypoechogen, hyperechogen, komplex) sowie der dorsalen Schallphänomene (keine, Schallverstärkung, Schallabschwächung) beurteilt werden. Die wesentlichen Charakteristika für benigne Herdläsionen sind eine dünne echoreiche Pseudokapsel, die parallele Ausrichtung bzw. ellipsoide Form, zarte Makrolobulierungen sowie ausgeprägte Hyperechogenität. 
Die wesentlichsten Charakteristika bösartiger Tumoren sind Spikulierungen, die antiparallele oder vertikale Orientierung, Unterbrechungen von Drüsen- und Bindegewebsstrukturen, Mikrolobulierungen, zentrale und dorsale Schallauslöschung sowie insbesondere der hyperechogene Randsaum [Delorme und Debus 1998]. Eine besondere Bedeutung kommt der Sonographie bei der Beurteilung von Zysten zu. Blande Zysten ohne Entartungsrisiko zeigen eine glatte, scharf begrenzte Wand, ein echoleeres Inneres sowie eine ausgeprägte dorsale Schallverstärkung. Komplizierte Zysten hingegen haben unregelmäßige verdickte Wandstrukturen, verdickte Binnensepten, solide Binnenläsionen oder Sedimentierungen. Die Bewertungskategorien (US-BI-RADS) entsprechen denen der Mammographie und der MRT.

Unabhängig hiervon kommt der Sonographie eine große Bedeutung bei der Durchführung perkutaner Biopsieverfahren und präoperativer Lokalisation zu. Da im Vergleich zu stereotaktischen oder MR-gesteuerten Interventionen geringere Kosten entstehen, ist sie die Methode der Wahl für interventionelle Eingriffe, wenn auffällige Läsionen zweifelsfrei in der Sonographie nachgewiesen werden können [Fornage 2000]. Bei der US-gesteuerten Stanzbiopsie werden mit Hochgeschwindigkeit und einer Nadelstärke zwischen 14 und 18 Gauge üblicherweise 3-5 Gewebeproben gewonnen. Die Feinnadelaspirationszytologie ist inzwischen nur noch der Punktion symptomatischer Zysten oder axillär auffälliger Lymphknoten vorbehalten [S3Leitlinie... Mammakarzinom 2008].

Variationen und Weiterentwicklungen der Mammasonographie betreffen u.a. die Dopplersonographie, bei der durch Kodierung visuelle und qualitative Darstellung der verschiedenen Geschwindigkeiten und Flussrichtungen möglich sind (Farbdoppler, Color Doppler Imaging (CDI)). Der Power-Doppler stellt ein neues Verfahren der farbkodierten Ultraschalldiagnostik dar, bei dem durch eine modifizierte Auswertung der Dopplersignale ein größerer Teil der Organdurchblutung dargestellt werden kann [Jörn und Rath 2000]. Die 3D/4D-Volumensonographie ist eine Methode, mit der man Ultraschallvolumendaten analysieren und darstellen kann. Sie bietet eine Zusatzeinstellung in der Mammadiagnostik bzw. Mammaintervention zur zweidimensionalen Sonographie [Weismann und Hergan 2007]. Neueste Techniken betreffen den automatisierten Volumen-Scan, bei dem die komplette Brust in 2-4 Einstellungen standardisiert durch eine MTRA sonographiert werden kann. Daten zum klinischen Stellenwert dieser Verfahren liegen bisher nicht vor. 


\subsection{Mamma-MRT}

Die Mamma-MRT wird üblicherweise an Ganzkörpermagneten mit einer Feldstärke zwischen 1,5 bis 3,0T durchgeführt. Zur Erzielung einer ausreichenden Ortsauflösung kommen bilaterale Oberflächenspulen, die sich an der Form der weiblichen Brust orientieren, zum Einsatz. Offene Spulen mit integriertem Kompressorium erlauben aktuell MRT-Untersuchungen in hoher Qualität. Die MRUntersuchung der Mamma erfolgt typischerweise einmalig vor sowie mehrmalig nach intravenöser Applikation eines paramagnetischen Kontrastmittels. Zum Einsatz kommen Kontrastmittel-sensitive T1-gewichtete Gradienten-Echo (GE)-Sequenzen, die mit einer Messdauer von weniger als zwei Minuten/Sequenz pro Schichtstapel eine ausreichend hohe zeitliche Auflösung erlauben.

Mit der Fähigkeit, mammographisch und sonographisch okkulte Mammakarzinome zu detektieren, hat sich die Mamma-MRT der Brust als bildgebende Zusatzmethode etabliert [Morris 2002]. Indikationen sind u.a. die Frage nach Multizentrizität/Multifokalität, Brustwandbeteiligung, Verlaufsuntersuchung während einer Chemotherapie, Tumorrezidive, Identifikation von okkulten Tumoren sowie die Früherkennung von Karzinomen in Hochrisikokollektiven [Morris 2002]. Insbesondere stellt die Mamma-MRT die Methode der Wahl dar in der Differenzierung zwischen Narbe und Rezidiv [Kuhl et al. 2007a; Orel 2008]. Wegen der Gefahr von falschpositiven Befunden sollte die Untersuchung in der 2. oder 3. Zykluswoche durchgeführt werden. Außerhalb dieser Zeitspanne kann es zu unspezifischen fokalen Kontrastmittelanreicherungen („Background-Enhancement $)$ kommen [Kuhl et. al 1997].

Die MRT der Mamma erfordert eine Nachbearbeitung der großen Anzahl von aquirierten Bildern. Die Bildsubtraktion erlaubt in diesem Zusammenhang eine zuverlässige Detektion kontrastmittelaufnehmender Bereiche, sofern keine Bewegungsartefakte oder hormonell stimulierte Areale mit gesteigerter Durchblutung vorliegen. Das Signal in auffälligen Läsionen kann semiquantitativ analysiert werden. Hierzu werden nach Platzierung einer geeigneten „region of interest" (ROI) entsprechende Signal-Zeit-Kurven software-gestützt berechnet. Auswertekriterien in der MRT betreffen die Form, Begrenzung, KM-Verteilung sowie dynamische Kriterien in der Früh- und Spätphase der Untersuchung. In Form des sog. Göttingen-Scores können die Befunde der einzelnen Kriterien bepunktet und in Form eines GesamtScores bewertet werden [Fischer et al. 2005]. In Abhängigkeit von der Gesamtpunktzahl ist weiterhin eine Zuordnung zu einer MRM-BI-RADS-Kategorie (Stufe 1-5) möglich [Fischer et al. 1993].

Die MRT der Brust unterscheidet Foki (Läsionen mit einem Durchmesser unter 5 $\mathrm{mm}$ ), Herdbefunde und Läsionen ohne raumfordernden Charakter (sog. „Non-masslike lesions"). 
Bei Herdläsionen werden die Form (rund, oval, lobuliert, irregulär), die Begrenzung (scharf begrenzt, irregulär, spikuliert) und das interne KM-Aufnahmeverhalten (homogen, heterogen, randförmig, dunkle interne Septen, KM-aufnehmende Septen, zentrales Enhancement) unterschieden. Nicht herdförmige Läsionen können fokal, linear, duktal, segmental, regional, diffus, heterogen, homogen, retikulär, symmetrisch oder asymmetrisch konfiguriert sein. Als assoziierte Veränderungen gelten Mamillen- oder Hautretraktion, Lymphadenopathie, Ödem, Invasion des Musculus pectoralis oder der Brustwand [American College of Radiology (ACR) 2003]. 


\section{Material und Methoden}

\subsection{Kollektiv}

Im Rahmen dieser retrospektiven Studie wurden die Daten von Patientinnen mit Mammakarzinomen im intraduktalen Stadium (DCIS) sowie invasiven Karzinomen bis zu einer Tumorgröße von 10mm (pT1a, pT1b), die im Zeitraum von 2003 bis 2011 im Diagnostischen Brustzentrum Göttingen untersucht wurden, ausgewertet. Bei allen Patientinnen lagen eine präoperativ angefertigte digitale VollfeldMammographie und eine KM-gestützte Mamma-MRT, die beide ausnahmslos im Brustzentrum angefertigt worden waren, vor. Extern durchgeführte Untersuchungen gingen in die Auswertung dieser Studie nicht ein. Die präoperativ im Brustzentrum Göttingen angefertigte Sonographie wurde berücksichtigt, sofern sie stattfand. Das Ziel dieser Auswertung war es, die Visualisierbarkeit von kleinen und frühen Mammakarzinomen mit den verschiedenen bildgebenden Verfahren zu evaluieren.

\subsection{Untersuchungsverfahren}

Die Mammographie erfolgte mit einem digitalen Vollfeldgerät (Senographe 2000D, Fa. General Electrics Medical Systems, Milwaukee, WI, USA), wobei in aller Regel 2 Untersuchungsebenen (kranio-kaudal [CC] und Schrägposition [MLO]) angefertigt wurden. Die MLO-Projektion wurde präferiert, sofern lediglich eine 1-EbenenMammographie durchgeführt wurde. Befundorientiert wurde diese Diagnostik im Einzelfall durch Tubuskompressions-und Vergrößerungsaufnahmen ergänzt.

Die sonographischen Befunde wurden mit handelsüblichen Geräten mit einem 11 MHz Schallkopf (Logiq E9, Fa. General Electrics Medical Systems, Milwaukee, WI, USA) erhoben.

Bei den Untersuchungen war eine lückenlose Durchuntersuchung der gesamten Brust gewährleistet. Als Untersuchungstechniken wurden alternativ die radiäre oder die mäanderförmige Technik gewählt. Die Untersuchungen wurden ausschließlich von den vier im Brustzentrum langzeittätigen Ärzten/Ärztinnen (Erfahrung in der Mammasonographie zwischen 12-28 Jahren) durchgeführt, so dass ein hohes Maß an Reproduzierbarkeit gewährleistet war.

Die KM-gestützte Mamma-MRT erfolgte an einem 1,5 Tesla Ganzkörpermagneten Magnetom Signa HDX (Fa. General Electrics Medical Systems, Milwaukee, WI, USA) unter Verwendung einer bilateralen offenen Oberflächenspule (Fa. Invivo) und einer spulenintegrierten Mammakompression (Fa. Noras). Die MRT-Untersuchungen wurden in allen Fällen dynamisch mit repetitiven Messungen vor und nach intravenöser Applikation eines paramagnetischen Kontrastmittels (Gadolinium-DTPA, Magnevist, Fa. Schering, Berlin) über einen Zeitraum von 8 Minuten nach KM-Gabe 
durchgeführt. Für die hierbei verwendete 3D-Technik wurde das Kontrastmittel in einer Dosierung von $0,1 \mathrm{mmol} / \mathrm{kg}$ Körpergewicht periphervenös appliziert. Es erfolgten Messungen mit T1-gewichteten Gradientenecho (GE)-Sequenzen (TR=8,4 $\mathrm{ms}, \mathrm{TE}=4,1 \mathrm{~ms}$, Flipwinkel $10^{\circ}$, FOV $380 \mathrm{~mm}$, Schichtdicke $2,5 \mathrm{~mm}$, Schichtanzahl 46, Spacing=0, Matrix 512x512, Messzeit: 1:26 min) mit 5 Repetitionen in axialer Angulierung. Zur Detektion vaskularisierter Veränderungen erfolgte obligat die

Subtraktion der Nativuntersuchung von der zweiten Messreihe nach Kontrastmittelapplikation. Zeigte sich in der Bildsubtraktion ein Bezirk mit vermehrter Kontrastmittelaufnahme, so schloss sich die semiquantitative Auswertung des Signalverhaltens, die so genannte "mean curve function" über die Zeit in einer geeigneten "region of interest" (ROI) an. Die ROI wurde hierbei durch die untersuchende MTRA in Größe und Lage derart gewählt, dass sie ein möglichst großes Areal mit maximaler Kontrastmittelanreicherung innerhalb einer auffälligen Läsion umfasste. Die Größe der ROI betrug in aller Regel 2 bis 5 Pixel. Regelhaft wurden darüber hinaus die Subtraktionsaufnahmen in MIP (,maximum intensity projection") -Technik zur dreidimensionalen Darstellung vermehrt anreichernder Gewebeareale erstellt.

Die Auswertung der Mammogramme und der Mamma-MRT erfolgten durch eine erneute Bildanalyse (Re-Analyse) im Rahmen dieser Dissertation durch einen erfahrenen Mammadiagnostiker (Uwe Fischer, Mammographieerfahrung >30 Jahre). Die Befunde der klinischen Untersuchung und der Mammasonographie wurden den jeweiligen Befundberichten entnommen.

\subsection{Befundauswertung}

Für die Auswertung wurde ein Auswertungsbogen entwickelt, der nachfolgend erläutert wird:

Zunächst wurde bei der Indikation zur Vorstellung im Diagnostischen Brustzentrum unterschieden, ob die Patientin wegen eines aufgefallenen Symptoms (Abklärungsdiagnostik) oder asymptomatisch im Rahmen der Früherkennung vorstellig wurde. Zu den Symptomen zählten u.a. unklare Knoten, neu aufgetretene Haut- oder Brustwarzeneinziehungen oder eine auffällige Sekretion. Das individuelle Risikoprofil (Eigenanamnese, Familienanamnese) ging nicht in die Evaluation ein.

Dem schriftlichen Befundbericht wurde entnommen, ob im Rahmen der klinischen Untersuchung (Inspektion, Palpation) durch den untersuchenden Arzt eine Auffälligkeit festgestellt worden war. 
Bei der Mammographie wurde die Bildqualität nach dem PGMI-System beurteilt und zwischen den Kategorien perfekt, gut, mäßig und ungenügend unterschieden. Ferner erfolgte eine Festlegung des mammographischen Dichtetyps gemäß den Ausführungen des American College of Radiology [American College of Radiology (ACR) 2003] in 4 Stufen (Tab.3.1a).

\begin{tabular}{l|l}
\hline Dichtetyp & Beschreibung \\
\hline ACR I & überwiegend lipomatös \\
ACR II & fibroglandulär \\
ACR III & inhomogen dicht \\
ACR IV & extrem dicht \\
\hline
\end{tabular}

Tab.3.1a: Definition des Dichtetyps im Mammogramm gemäß ACR [American College of Radiology (ACR) 2003]

Anschließend wurden die Mammogramme in Anlehnung an die BI-RADSKategorisierung des American College of Radiology [American College of Radiology (ACR) 2003] evaluiert und in 5 Kategorien unterteilt (B1-B5) (Tab.3.1.b).

\begin{tabular}{ll}
\hline BI-RADS-Kategorisierung des ACR & Befund \\
\hline BI-RADS 1 & unauffällig \\
BI-RADS 2 & benigne \\
BI-RADS 3 & $\begin{array}{l}\text { wahrscheinlich benigne, unklar, Kontrolle } \\
\text { empfohlen }\end{array}$ \\
BI-RADS 4 & suspekt, Abklärung durch Biopsie erforderlich \\
BI-RADS 5 & hochgradig karzinomverdächtig \\
\hline
\end{tabular}

Tab.3.1b: BI-RADS-Kategorisierung des ACR [American College of Radiology (ACR) 2003]

Lag mammographisch eine Auffälligkeit der Kategorie BI-RADS III, IV oder V vor, so erfolgte eine Deskription der Art des Befundes nach den Kriterien „Verdichtung“, „Herdbefund“, „Architekturstörung“ oder „Verkalkung“.

Bei den sonographischen Auswertungen wurde die Gewebetextur der Brust hinsichtlich ihrer Homogenität und dem Vorliegen störender zystischer 
Veränderungen begutachtet und kategorisiert. Dies erfolgte nach den Kriterien homogen, homogen- fettig und fibrozystisch- mastopathisch.

Die Kategorisierung der sonographischen Untersuchung bzw. Befunde erfolgte ebenfalls in Anlehnung an die BI-RADS-Klassifikation in 5 Stufen (Tab.3.1b).

Die Evaluation der Mamma-MRT umfasste vorab die Verschlüsselung der Artefaktstufe in 4 Graduierungen [Fischer et al. 2005] (Tab.3.1c).

\begin{tabular}{l|l}
\hline MRM-Artefaktstufen & \\
\hline MRM-Artefaktstufe 1 & Keine Bewegungs-/Subtraktionsartefakte \\
MRM-Artefaktstufe 2 & Geringe Bewegungs-/Subtraktionsartefakte \\
MRM-Artefaktstufe 3 & ausgeprägte Bewegungs-/Subtraktionsartefakte \\
MRM-Artefaktstufe 4 & inakzeptable Bewegungs-/Subtraktionsartefakte \\
\hline
\end{tabular}

Tab.3.1c: MRM-Artefaktstufen [Fischer et al. 2005]

Die Auswertung des MR-Dichtetyps in der 2. Messung nach KM-Gabe erfolgte ebenfalls in 4 Stufen gemäß entsprechender Definition [Fischer et al. 2005] (Tab.3.1d).

\begin{tabular}{l|l}
\hline MRM-Dichtetyp & \\
\hline MRM Dichtetyp 1 & kein Enhancement des Parenchyms \\
MRM Dichtetyp 2 & fleckiges Enhancement des Parenchyms \\
MRM Dichtetyp 3 & fleckig-flächiges Enhancement des Parenchyms \\
MRM Dichtetyp 4 & extrem starkes Enhancement des Parenchyms \\
\hline
\end{tabular}

Tab.3.1d: MRM-Dichtetyp [Fischer et al. 2005]

Die Befundung der Mamma-MRT erfolgte in Anlehnung an das ACR in den Kategorien BI-RADS 1-5 (Tab.3.1.b). Bei auffälligen Befunden in der MRT wurde vermerkt, ob es sich um einen Fokus, eine Herdsetzung oder eine nichtraumfordernde Läsion (Non-mass-like Lesion) handelte. Lag ein Herd vor, so wurde eine multimodale Evaluation nach dem sog. Göttingen-Score durchgeführt [Fischer et al. 2005] (Tab.3.1e). 


\begin{tabular}{llll}
\hline $\begin{array}{l}\text { MRM-Punkteschema } \\
\text { Göttingen Score }\end{array}$ & & & \\
\hline Punkte & 0 & 1 & 2 \\
Form & rund, oval & irregulär, dendritisch & - \\
Begrenzung & scharf & unscharf & - \\
KM-Verteilung & homogen & inhomogen & Ringenhancement \\
initialer Anstieg & $<50 \%$ & $50 \%-100 \%$ & $>100 \%$ \\
postinitialer & Anstieg & Plateau & wash-out \\
Signalverlauf & & & \\
\hline
\end{tabular}

Tab. 3.1e: MRM Punkteschema (Göttingen Score) [Fischer et al. 2005]

In den präsentierten Daten wurden die BI-RADS-Kategorie 1und 2 der Gruppe falsch-negativ und die BI-RADS-Kategorie 4 und 5 der Gruppe richtig-positiv zugeordnet. Die BI-RADS-Kategorie 3 wurde in der Auswertung als falsch-positiv bewertet bzw. wurde ausdrücklich vermerkt, wenn sie als richtig-positiv eingeschätzt wurde.

\section{Biopsieverfahren}

Perkutane Stanz- oder Vakuumbiopsien wurden hinsichtlich der eingesetzten bildgebenden Methode (Ultraschall, Mammographie oder MRT) festgehalten.

\section{B-Klassifikation}

Die in der histopathologischen Untersuchung ermittelten Befunde wurden gemäß der histopathologischen B-Klassifikation für perkutan-bioptisch entnommene Gewebeproben entsprechend der National Coordinating Group for Breast Screening Pathology (NHSBSP) [Sloane et al. 1994] erfasst (Tab.3.1f). 


\begin{tabular}{|c|c|}
\hline B-Kategorie & Beschreibung \\
\hline $\mathrm{B} 1$ & $\begin{array}{l}\text { Nicht verwertbar oder ausschließlich } \\
\text { Normalgewebe }\end{array}$ \\
\hline B2 & $\begin{array}{l}\text { Benigne, u. a. fibrös-zystische Mastopathie, } \\
\text { Fibroadenom, sklerosierende Adenose, } \\
\text { periduktale Mastitis }\end{array}$ \\
\hline B3 & $\begin{array}{l}\text { Benigne, aber mit unsicherem biologischen } \\
\text { Potential u. a. atypische intraduktale } \\
\text { Epithelproliferationen, bei denen eine definitive } \\
\text { Festlegung an der perkutanen Biopsie nicht } \\
\text { möglich ist (z. B. atypische duktale Hyperplasie: } \\
\text { in Abhängigkeit von Ausdehnung und Grad der } \\
\text { Atypie ggf. auch Kategorie B4); atypische } \\
\text { lobuläre Hyperplasie und LCIS; papilläre } \\
\text { Läsionen (bei hochgradigem V. a. papilläres } \\
\text { DCIS: ggf. auch Kategorie B4); radiäre } \\
\text { Narbe/komplexe sklerosierende Läsion; V. a. } \\
\text { Phylloides-Tumor; }\end{array}$ \\
\hline B4 & $\begin{array}{l}\text { Malignitätsverdächtig } \\
\text { z. B. vermutlich maligne Veränderung, aber } \\
\text { Beurteilung aus technischen Gründen } \\
\text { eingeschränkt; atypische intraduktale } \\
\text { Epithelproliferationen in Abhängigkeit von } \\
\text { Ausdehnung und Schwere der Atypie (siehe } \\
\text { auch Kategorie B3) }\end{array}$ \\
\hline B5 & $\begin{array}{l}\text { Maligne } \\
\text { z. B. DCIS, invasive Karzinome, maligne } \\
\text { Lymphome } \\
\text { B5a = nicht-invasives Mammakarzinom } \\
\text { B5b = invasives Mammakarzinom } \\
\text { B5c = fraglich invasives Karzinom } \\
\text { B5d = maligner Tumor, nicht primär Mamma }\end{array}$ \\
\hline
\end{tabular}

Tab.3.1f: Histopathologische B-Klassifikation entsprechend der National Coordinating Group for Breast Screening Pathology (NHSBSP) [Sloane et al. 1994]

Histologisch wurden die B3-Läsionen in verschiedene Gruppen: Papillome, ADH, LIN und radiäre Narbe eingeteilt. DCIS, IDC und ILC wurden jeweils nach Grading-Score 1-3 [nach Bässler et al. 1992] unterschieden. Tubuläre, muzinöse, medulläre, papilläre und andere Karzinome wurden, wie auch der M. Paget, unterschieden und dokumentiert. Allerdings wurden diese Tumorentitäten in unserer statistischen Auswertung nicht berücksichtigt.

Die histopathologische post-operative TNM-Klassifkation erfolgte nach Singletary et al. [Singletary et al. 2002]. Zusätzlich wurde vermerkt, ob die Methode der „Sentinel Node Biopsy" (SNB) angewendet wurde oder nicht. Ferner wurden der Rezeptorstatus, eine potentielle intraduktale Tumorkomponente („extensive intraductal component", EIC), Multifokalität (MFC), Multizentrizität (MCC) und Bilateralität eines Mammakarzinoms erfasst. Letztendlich wurden auch diese Parameter in den Auswertungen nicht berücksichtigt. 
Die Zielsetzung dieser Auswertung betraf die Frage, mit welcher Sensitivität jedes der drei etablierten bildgebenden Untersuchungsverfahren (Mammographie, Ultraschall, Mamma-MRT) das frühe bzw. kleine Mammakarzinom im Stadium pTis, pT1a und pT1b nachweist. Hierbei wurde in dem präselektionierten Patientengut, das sich ausschließlich aus Patienten mit gesicherten Karzinomen in den o.g. Stadien zusammensetzte, die Gesamtzahl an diagnostizierten Karzinomen als 100\% gewertet. Die Berechnung der Sensitivität erfolgte nach der Gleichung Rpos : (Rpos + Fneg), wobei Rpos für die Anzahl der richtig-positiven Befunde und Fneg für die Anzahl der falsch-negativen Befunde in der Bildgebung steht. Darüber hinaus wurde untersucht, welche typischen Befunde in der Bildgebung für das jeweilige Untersuchungsverfahren im Nachweis des kleinen bzw. frühen Mammakarzinoms führend waren. 


\section{Ergebnisse}

\subsection{Kollektiv}

In einem Zeitraum von Mai 2003 bis Januar 2011 wurden im Diagnostischen Brustzentrum Göttingen bei 1789 Patienten unklare Befunde in der Brust perkutanbioptisch abgeklärt. Histologisch ergaben sich hierbei benigne Läsionen (histologischer Befund der Kategorien B1 und B2) in 912 Fällen (51\%). Läsionen mit unklarem biologischem Potential (histologischer Befund der Kategorie B3) lagen in 179 Fällen (10\%) vor.

Diese teilten sich in absteigender Häufigkeit auf in papilläre Läsionen (40\%), radiäre Narben $(20 \%)$, ADH (15\%), lobuläre Neoplasien (15\%), phylloide Tumoren (2\% und andere $8 \%$ ). 698 (39\%) der perkutan-bioptischen Läsionen waren maligne, davon 163 (23\%) intraduktal (histologischer Befund der Kategorien B5a) und 535 (77\%) invasiv (histologischer Befund der Kategorien B5b). In der Gruppe der benignen Befunde wurden $5(0,5 \%)$ Patientinnen einer zusätzlichen operativen Abklärung zugeführt. In der Gruppe der Frauen mit unklarem biologischem Potential war dies bei $86(48 \%)$ der Fall. Alle 698 Patientinnen mit einem malignen Tumor wurden operiert. Anhand der endgültigen histologischen Aufarbeitung nach operativer Befundentfernung fanden sich 103 Patientinnen mit einem Mammakarzinom in einem frühen Tumorstadium (pTis, pT1a, pT1b). Hinzu kamen 6 Frauen, die primär einer offenen Biopsie unterzogen wurden und bei denen sich ebenfalls ein Karzinom im frühen Stadium fand. Diese 109 Patientinnen, bei denen eine präoperative Mammographie und ein MRT vorlagen, stellen das ausgewertete Kollektiv dieser Studie dar (Abb.4.1a). Der Anteil der Patientinnen mit einer intraduktalen Tumorausdehnung (DCIS) betrug hierbei 50 (46\%). Mit Blick auf das Grading waren hierunter 13 low-grade-DCIS (G1), 14 intermediate-DCIS (G2) und 23 high-gradeDCIS (G3). In der Gruppe der invasiven Karzinome fand sich das Stadium pT1a bei 17 Frauen (16\%) und das Stadium pT1b bei 42 Frauen (38\%) (Abb. 4.1.a). Eine präoperative Sonographie lag bei 103 der Patienten vor, hierunter waren 45 Patienten mit DCIS, 17 mit einem Tumor im Stadium pT1a und 41 Patienten mit einem Tumor im Stadium pT1b. 


\section{Anzahl DCIS, pT1a und pT1b; $n=109$}

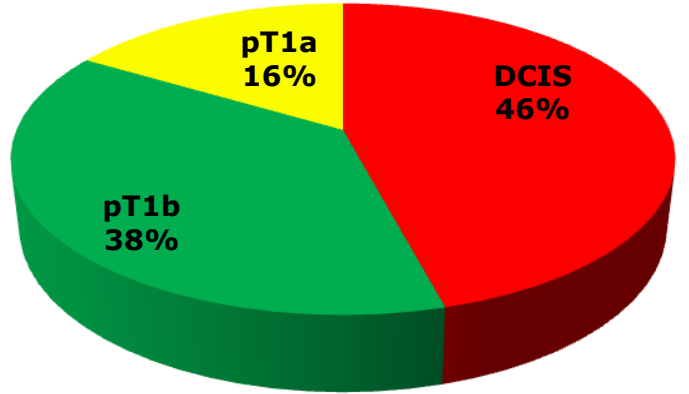

Abb.4.1a: Verteilungsmuster der Tumorstadien (pTis, pT1a, pT1b) bei 109 Patientinnen mit Mammakarzinom im Frühstadium

Die Altersverteilung der 109 Patienten mit einem früh erkannten Brustkrebs reichte von 26 bis 79 Jahren, im Mittel betrug sie 57,1 Jahre. 88 Patienten stellten sich im Rahmen der Früherkennung und 21 Patienten im Rahmen der Abklärung eines auffälligen Symptoms vor. 7 Frauen des Kollektivs wurden im Rahmen der Tumornachsorge untersucht.

Bei der klinischen Untersuchung fanden sich bei 24 von 109 Patientinnen (22\%) Auffälligkeiten. 85 Patientinnen waren klinisch unauffällig, tumorassoziierte Veränderungen konnten daher hier nur bildgebend und histologisch verifiziert werden (Abb.4.1b).

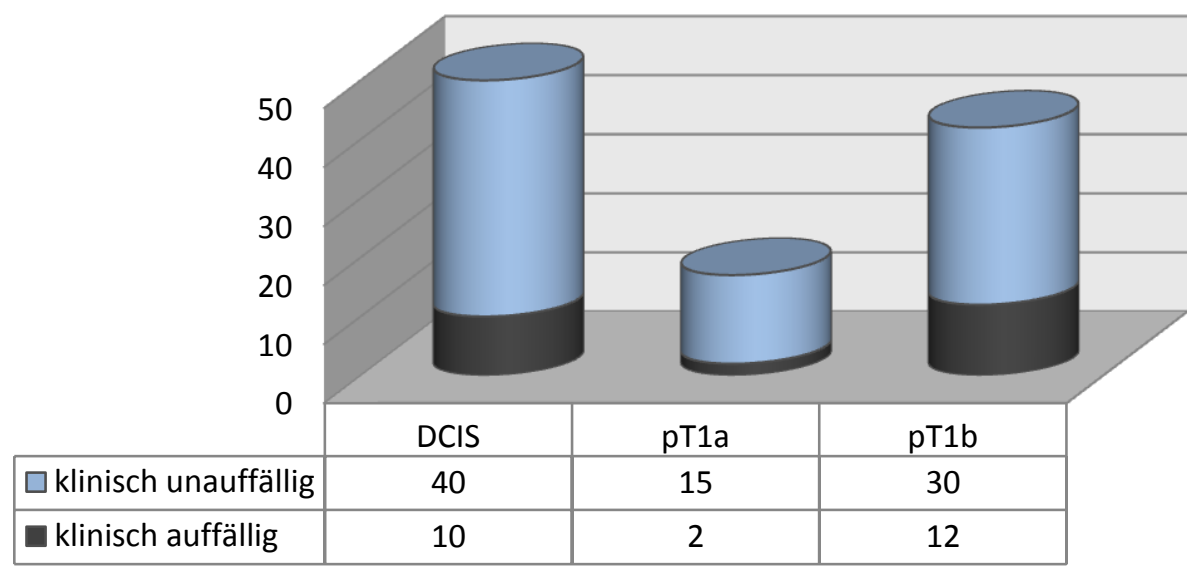

Abb.4.1b: Klinische Auffälligkeiten bzw. Unauffälligkeiten in der Gruppe der DCIS, pT1a - und pT1b-Gruppe in absoluten Zahlen 


\subsection{Ergebnisse Mammographie}

Die Qualität der Röntgenmammographie nach dem PGMI-System zeigte fast ausnahmslos eine perfekte (57\%) oder gute Qualität (42\%). Dies entsprach einer Graduierung nach Stufe I gemäß den deutschen Empfehlungen in 99\% der Fälle. Lediglich 1\% der Aufnahmen war "moderat" (entsprechend Stufe II im nationalen System). Inadäquate Bilder (Stufe III) lagen im Kollektiv nicht vor (Abb.4.2a).

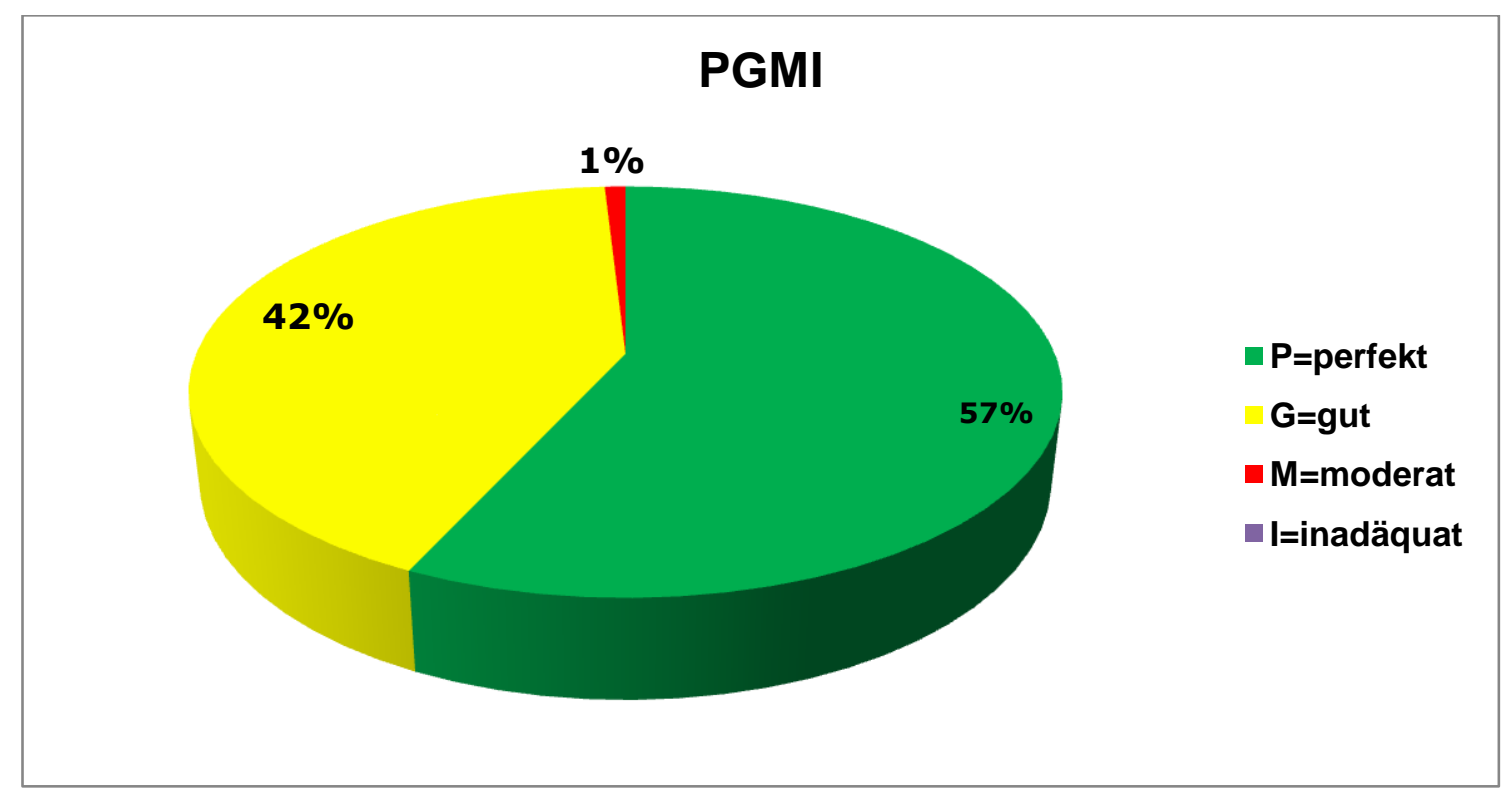

Abb.4.2a: Verteilung der Qualitätsstufen der Röntgenmammographie nach dem PGMI-System bei 109 Patientinnen mit Mammakarzinom im Frühstadium

Die Evaluation der Dichte zeigte, dass der größte Teil der Patientinnen (43\%) einen Dichtetyp III (nach ACR) aufwies. 37\% Patientinnen zeigten einen Dichtetyp ACR IV. Transparentere Parenchymstrukturen (Dichtetypen ACR I und ACR II) lagen bei jeder 5. Patientin vor (Abb.4.2b). 


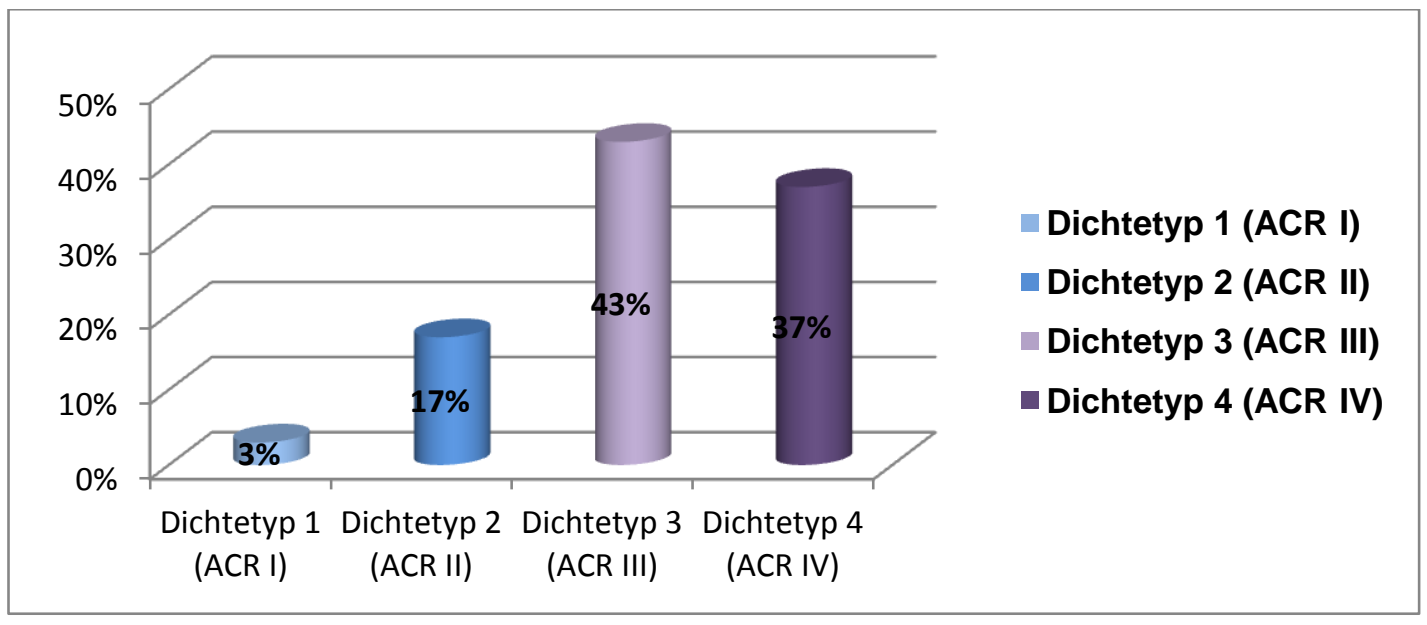

Abb.4.2b: Verteilung des Dichtetyps gemäß American College of Radiology (Typ ACR I-IV) in der Mammographie bei 109 Patientinnen mit Mammakarzinom im Frühstadium

Die Abb.4.2c zeigt den prozentualen Anteil an falsch-negativen bzw. richtig-positiven Befunden innerhalb der Dichtetypgruppen ACR I-IV. Zu den falsch-negativen wurden die BI-RADS-Gruppen 1-3 gerechnet, zu den richtig-positiven die BI-RADS-Gruppen 4 und 5. Deutlich wurde die Abnahme an richtig-positiven Befunden mit Zunahme der Dichtegruppe und im Umkehrschluss die Zunahme an falsch-negativen Befunden bei steigendem Dichtetyp.

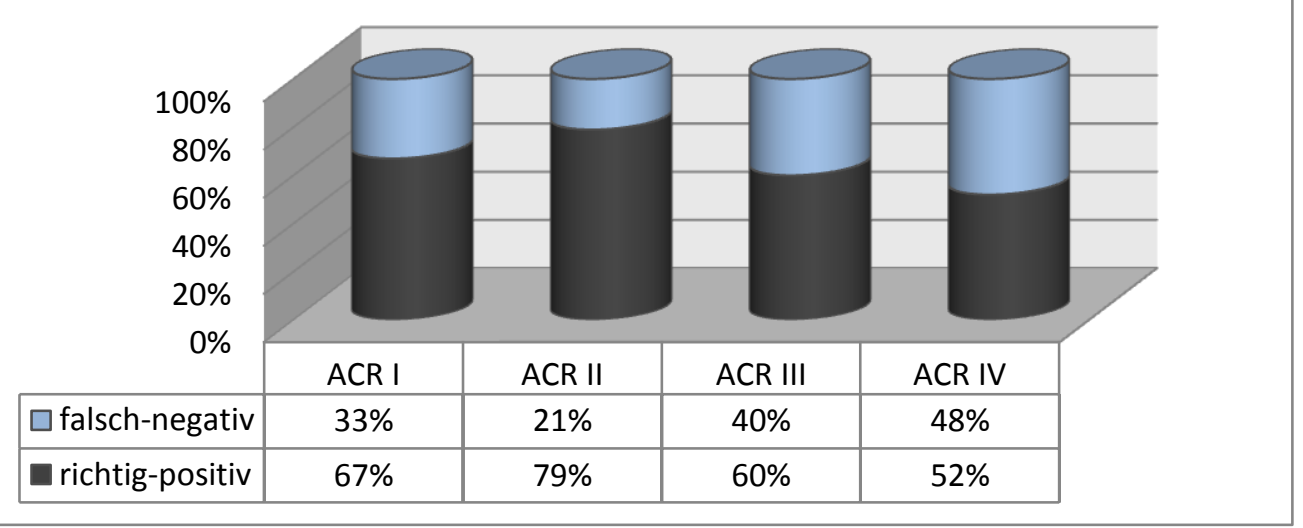

Abb.4.2c: Prozentuale Verteilung der richtig-positiven (BI-RADS 4+5) bzw. falsch-negativen (BIRADS 1-3) Befunde innerhalb der ACR-Gruppe I-IV 
Im untersuchten Patientenkollektiv zeigte sich etwa ein Drittel aller Karzinome mammographisch okkult oder unsuspekt. Sie wurden als Befunde der Kategorie BIRADS 1 (26\%) oder BI-RADS 2 (8\%) verschlüsselt. Der Anteil der unklaren und durch Verlauf zu kontrollierenden Befunde der Kategorie BI-RADS 3 lag bei $6 \%$ (Abb.4.2d). Knapp zwei Drittel wiesen auf Malignität verdächtige Veränderungen auf (BI-RADS 4: 39\%; BI-RADS 5: 21\%).

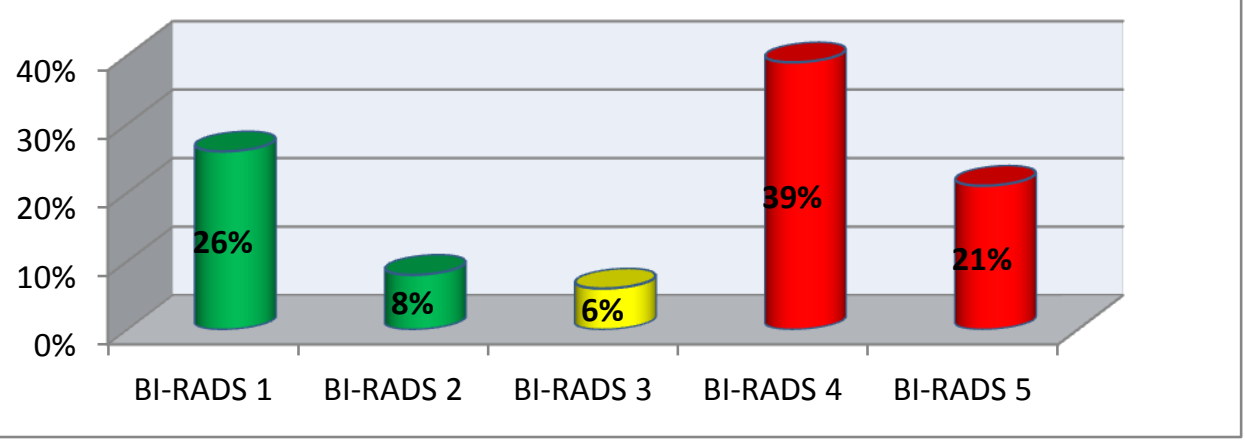

Abb.4.2d: Kategorisierung mammographischer Befunde bei 109 Patientinnen mit Mammakarzinom im Frühstadium nach MX-BI-RADS 1-5

Innerhalb der Patienten mit auffälligem Befund in der Mammographie (BI-RADS 4: 42 Patientinnen; BI-RADS 5: 23 Patientinnen) zeigte die detaillierte Aufteilung der histopathologischen Befunde jeweils einen sehr hohen Anteil an intraduktalen Tumorformen (74\%). Die invasive pT1a-Gruppe war mit 53\%, die pT1b-Gruppe mit $45 \%$ vertreten (Abb.4.2e).

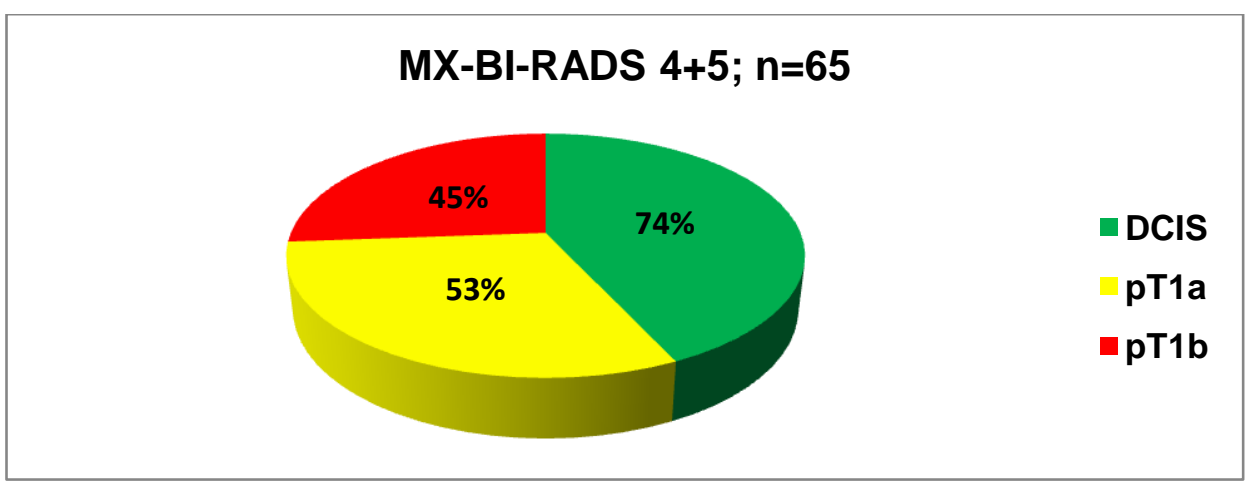

Abb. 4.2e: Verteilung von DCIS, pT1a und pT1b in den Gruppen MX-BI-RADS 4 und 5 
Das DCIS zeigte sich in 68\% mammographisch führend durch Mikroverkalkungen. Das intermediate-DCIS führte hier mit 11 von 14 (79\%), gefolgt vom high-gradeDCIS mit 18 von 23 (78\%). Das low-grade-DCIS zeigte sich mit 8 von 13 Fällen (62\%). Verdichtungen, Herde und Architekturstörungen spielten prozentual nur eine sehr geringe Rolle. $26 \%$ der DCIS waren mammographisch okkult (Abb. 4.2f).

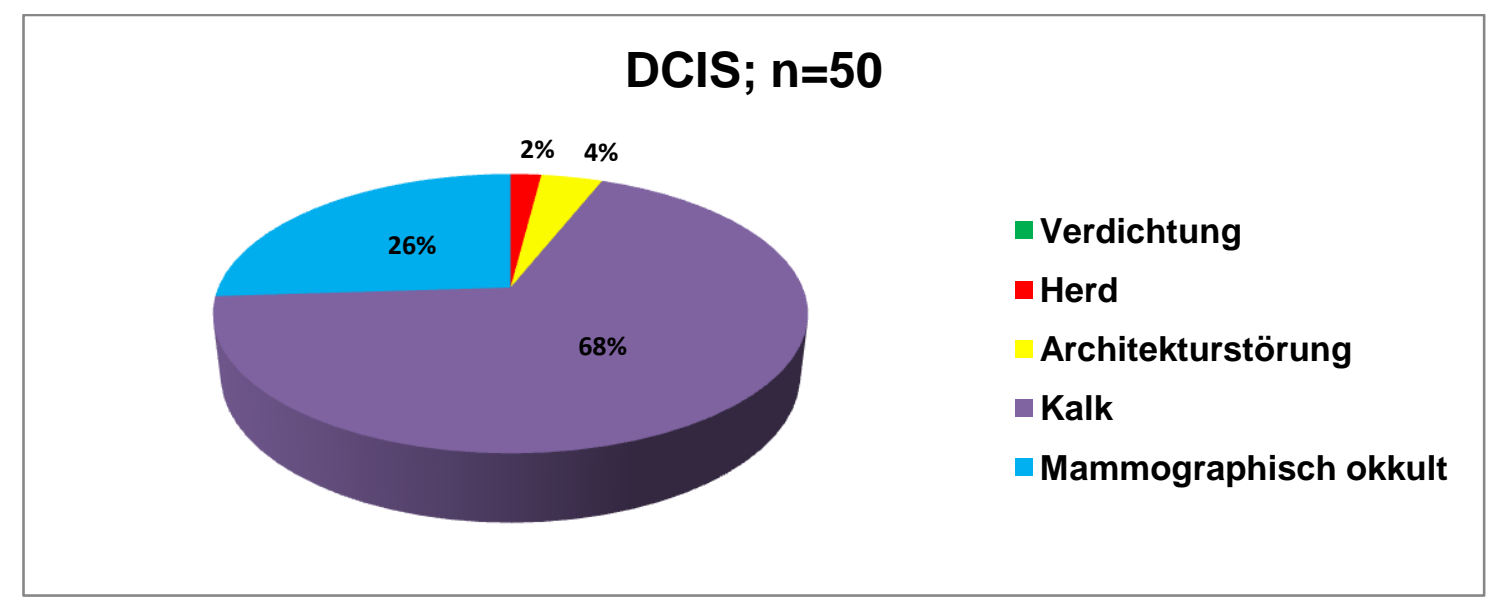

Abb.4.2f: Führendes Erscheinungsbild des DCIS im Mammogramm

In der Gruppe der pT1a-Tumoren waren insgesamt 8 der 17 (47\%) Karzinome mammographisch okkult. 33\% zeigten Kalkabsonderungen. Herdbefunde wiesen in $10 \%$ auf die korrekte Diagnose. Verdichtungen und Architekturstörungen waren gleichwertig mit jeweils $5 \%$ nachzuweisen (Abb.4.2g).

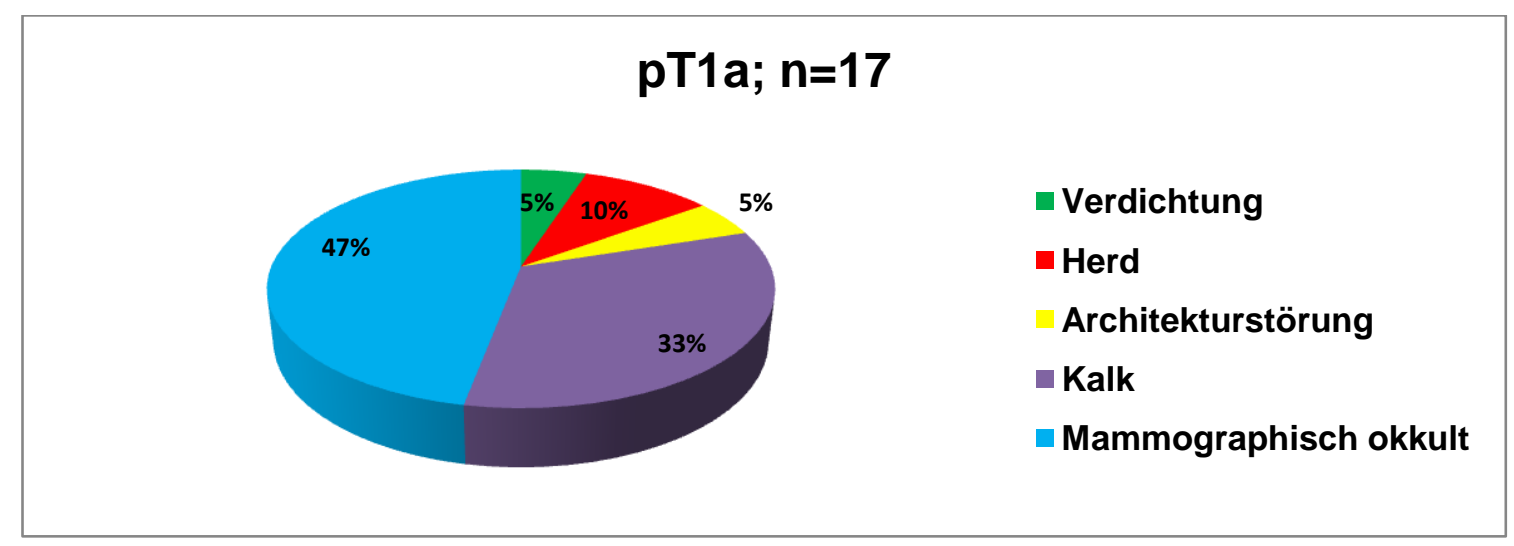

Abb.4.2g: Anzahl an Verdichtungen, Herdbefunden, Architekturstörungen und Mikroverkalkungen bei Karzinomen im Stadium pT1a (Mammographie) 
In der Gruppe der Tumoren im Stadium pT1b konnten 19 der 42 (45\%) Karzinome mammographisch erkannt werden. 30\% der Befunde imponierten als Herde und in $11 \%$ der Fälle dominierten Kalkabsonderungen. Verdichtungen und Architekturstörungen lagen nur in wenigen Fällen vor (Abb.4.2h).

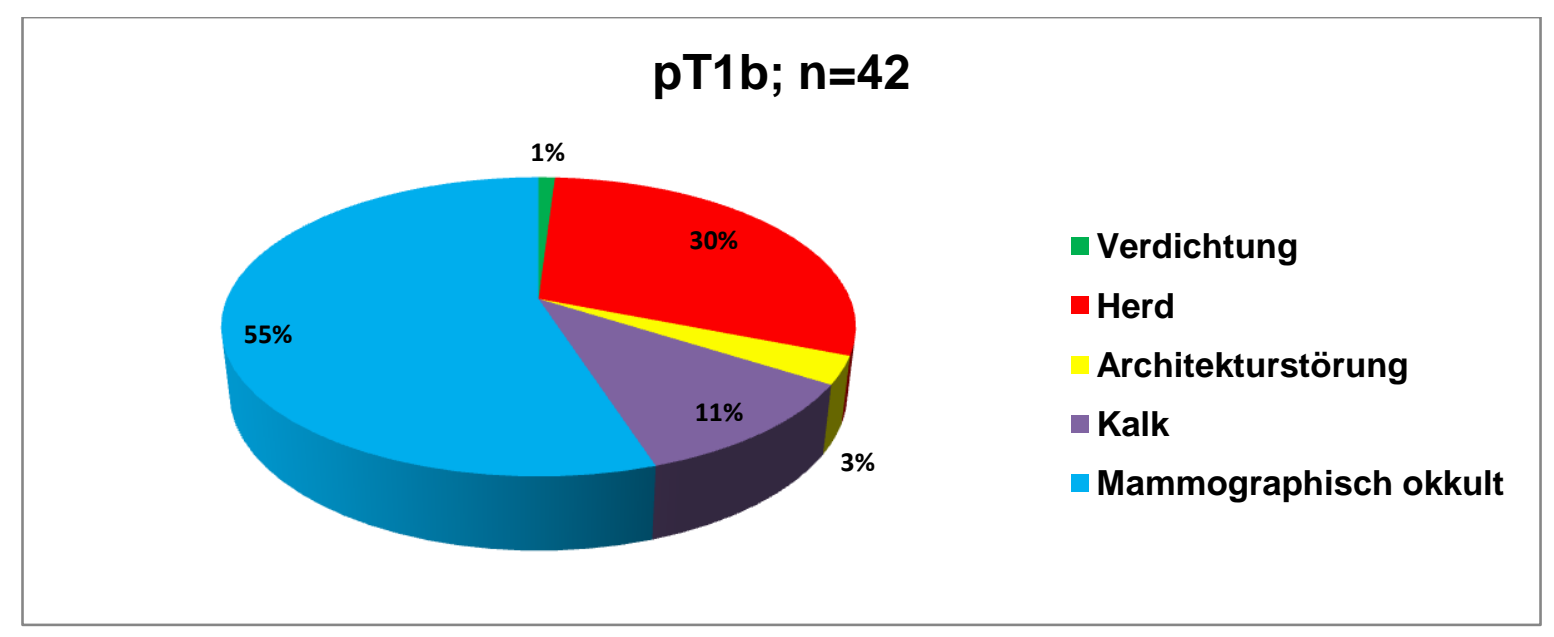

Abb.4.2h: Anzahl an Verdichtungen, Herdbefunden, Architekturstörungen und Mikroverkalkungen bei Karzinomen im Stadium pT1b (Mammographie)

Für alle früh erkannten Tumoren zeigt Abb.4.2i die Verteilung des jeweiligen Hauptbefundes (Kalk, Architekturstörung, Herd und Verdichtung) in der Mammographie. Low-grade-DCIS wurde ausschließlich anhand des Kriteriums „Mikrokalk“ nachgewiesen. In der intermediate-type-DCIS-Gruppe waren es $82 \%$ und in der Gruppe der high-grade-DCIS 95\%. 


\begin{tabular}{|c|c|c|c|c|c|c|}
\hline \multirow[t]{2}{*}{$\begin{array}{r}100 \% \\
80 \% \\
60 \% \\
40 \% \\
20 \% \\
0 \%\end{array}$} & & & & & & \multirow{6}{*}{$\begin{array}{l}\text { Kalk } \\
\text { Architekturstörung } \\
\text { Herd } \\
\text { Verdichtung }\end{array}$} \\
\hline & $\begin{array}{c}\text { DCIS } \\
\text { G1 }\end{array}$ & $\begin{array}{c}\text { DCIS } \\
\text { G2 }\end{array}$ & $\begin{array}{c}\text { DCIS } \\
\text { G3 }\end{array}$ & pT1a & pT1b & \\
\hline Kalk & $100 \%$ & $82 \%$ & $95 \%$ & $60 \%$ & $29 \%$ & \\
\hline Architekturstörung & $0 \%$ & $18 \%$ & $0 \%$ & $10 \%$ & $8 \%$ & \\
\hline Herd & $0 \%$ & $0 \%$ & $5 \%$ & $20 \%$ & $59 \%$ & \\
\hline Verdichtung & $0 \%$ & $0 \%$ & $0 \%$ & $10 \%$ & $4 \%$ & \\
\hline
\end{tabular}

Abb.4.2i: Mammographische Hauptbefunde (Kalk, Architekturstörung, Herd und Verdichtung) der DCIS-Tumoren in Abhängigkeit von deren Grading (G1-3), sowie der pT1a- und pT1bKarzinome

Die folgende Abbildung (Abb.4.2j) zeigt die mammographisch falsch-negativen Befunde in absoluten Zahlen in der Übersicht.

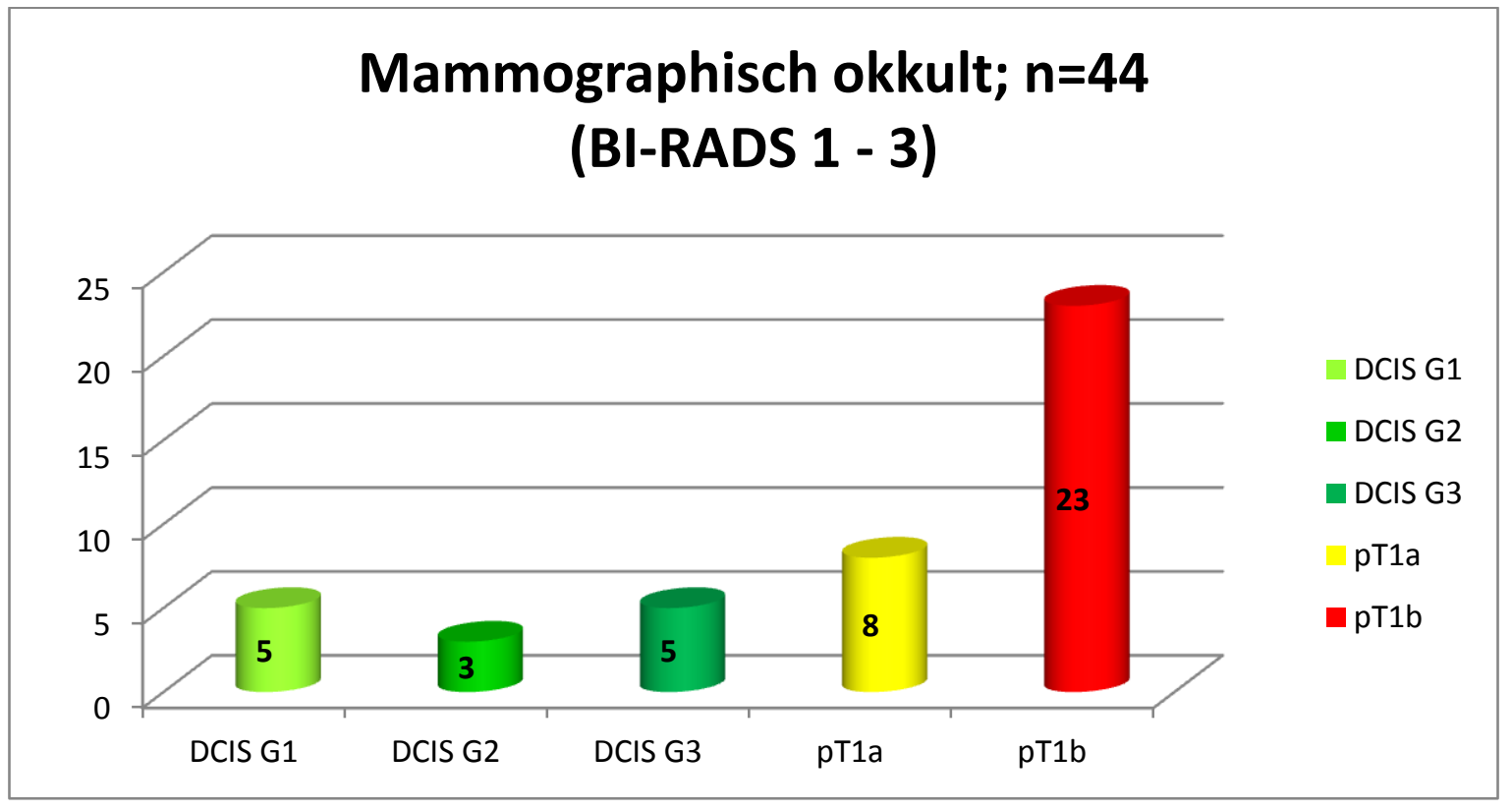

Abb.4.2j: Übersicht der gesamten mammographisch okkulten Befunde in absoluten Zahlen 


\subsection{Ergebnisse Ultraschall}

Das präoperativ ermittelte sonographische Kollektiv enthielt insgesamt 103 Patientinnen. Bei der Auswertung wurde zunächst der Texturtyp bzw. die Struktur der Brustdrüse evaluiert. Hierbei zeigte sich, dass der überwiegende Strukturtyp der Brüste des Patientenkollektivs „homogen parenchymal“ (HP: 46\%) bzw. „inhomogen“ (I: 44\%) war. Der Strukturtyp "homogen lipomatös (HF: 7\%)“ bzw. „fibrös-zystische Mastopathie" (FZM: 3\%) spielte nur eine untergeordnete Rolle (Abb.4.3a).

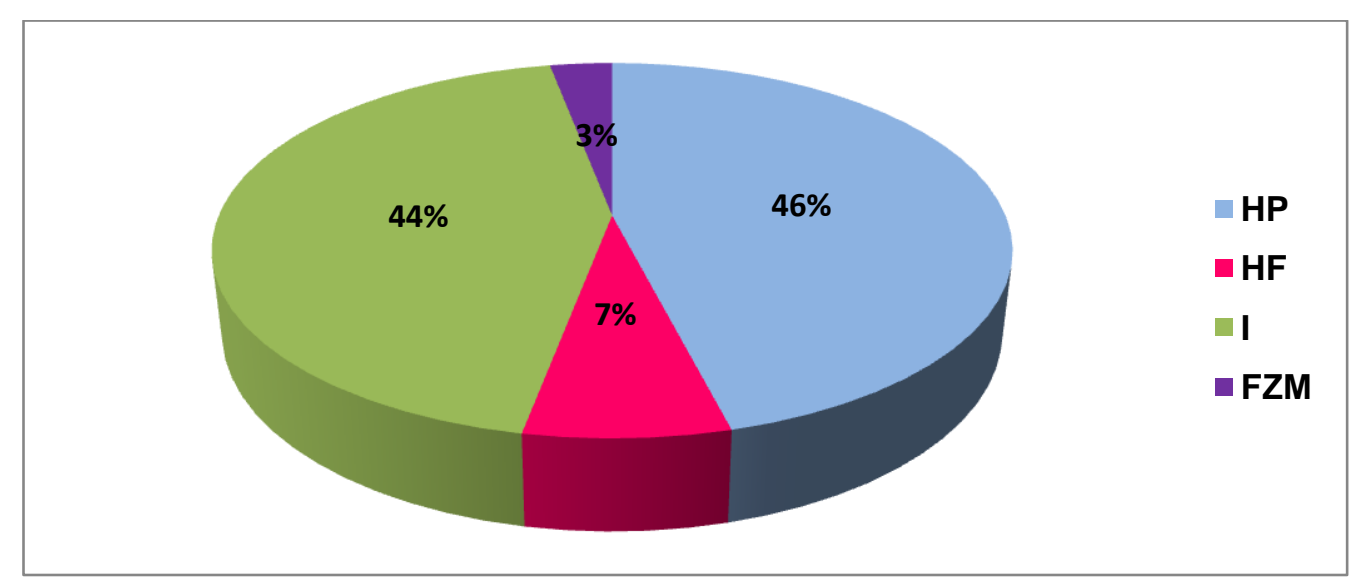

Abb.4.3a: Texturtyp der Mamma im Sonogramm (HP: homogen parenchymal; HF: homogen lipomatös; I: inhomogen; FZM: fibrös-zystische Mastopathie)

Die Abb. 4.3b zeigt die falsch-negativen bzw. die richtig-positiven Befunde innerhalb der verschiedenen Texturtypen der Mamma im Sonogramm. Innerhalb der fibröszystisch-mastopathischen Gruppe wurden in 100\% falsch-negative Befunde eruiert. In der homogen parenchymalen Gruppe waren es nur noch $64 \%$ der falschnegativen Befunde. 


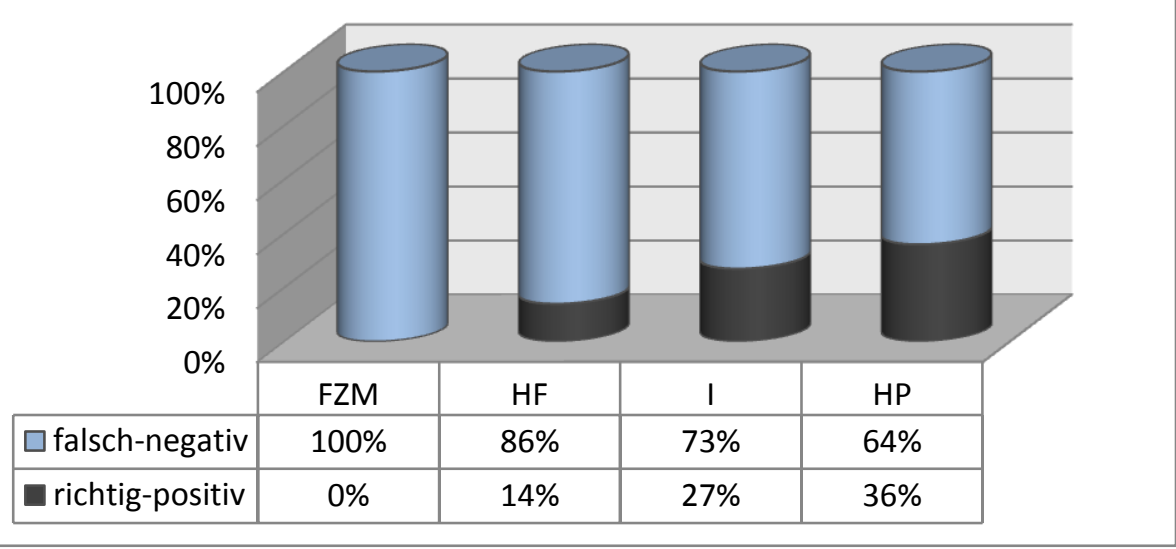

Abb. 4.3b: falsch-negative bzw. richtig-positive Befunde innerhalb der verschiedenen Texturtypen der Mamma im Sonogramm (FZM: fibrös-zystische Mastopathie HF: homogen lipomatös; I: inhomogen; HP: homogen parenchymal)

Die sonographische Auswertung zeigte in $50 \%$ einen Befund der Kategorie US-BIRADS 1 und in 11\% der US-BI-RADS 2, was laut der Nomenklatur einem unauffäligen Normalbefund entspricht, auf. Vorausgesetzt, man rechnet die BIRADS-Kategorie 3 zu den richtig-positiven Befunden, entfiel hier ein Anteil von 12\% auf diese Kategorie. Bei Ausschluss der US-BI-RADS-Kategorie 3 wären es dann nur noch $61(60 \%)$. In den sicher tumorverdächtigen und sicher erkannten Kategorien BIRADS 4 und 5 zeigte sich hier eine prozentuale Gesamtzahl von 27\%, wobei die USBI-RADS-Kategorie 4 mit 17\% sicherer eruiert wurde (Abb.4.3c).

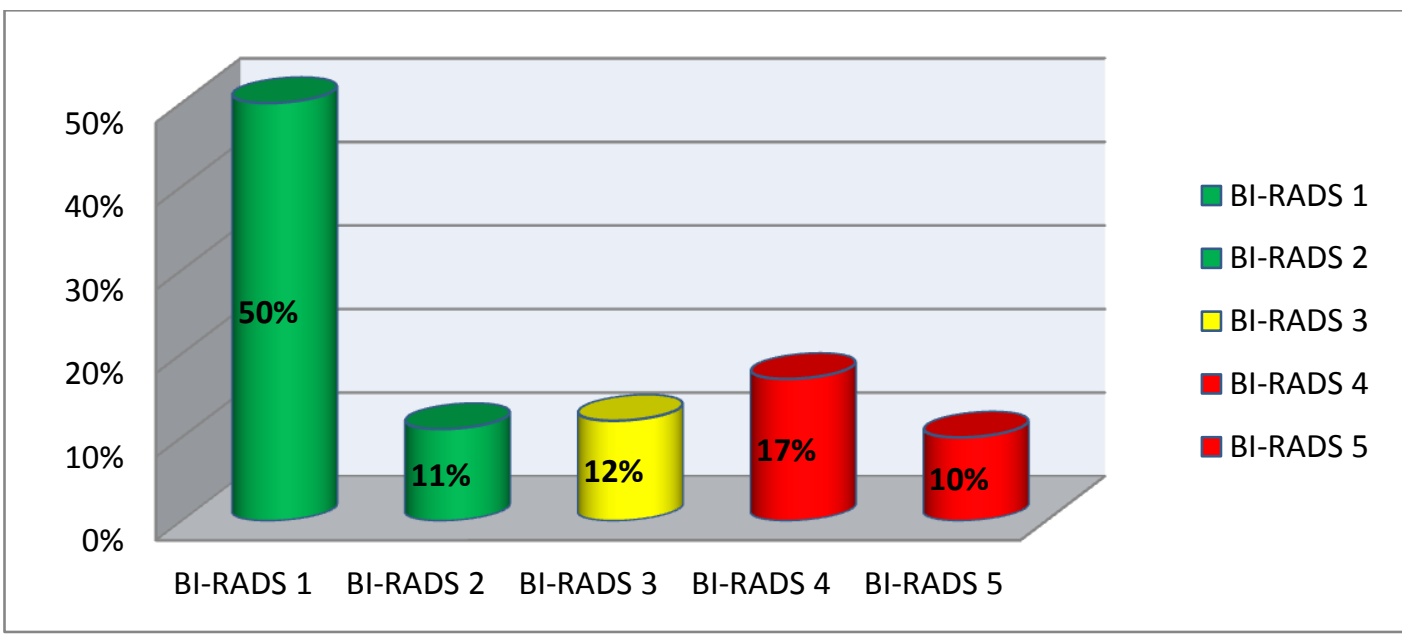

Abb.4.3c: Kategorisierung sonographischer Befunde nach US-BIRADS 1-5 ( $n=103)$ 
Abb.4.3d zeigt den Anteil der falsch-negativen Befunde im Ultraschall. Hierbei wurden die US-Kategorien BI-RADS 1, 2 und 3 kombiniert. Insgesamt 63 Fälle (61\%) wurden sonographisch nicht erkannt. Das sind $73 \%$ aller DCIS, $71 \%$ aller pT1aTumore und $44 \%$ aller pT 1 b-Tumore.

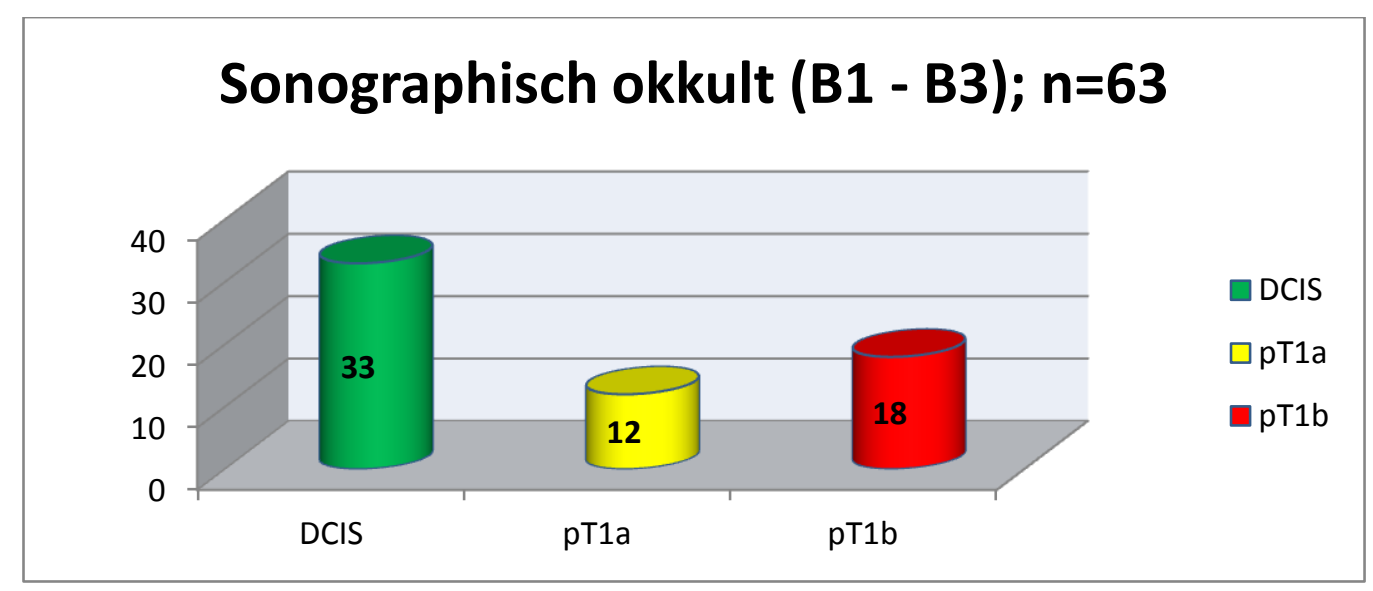

Abb.4.3d: Anteil an DCIS, pT1a und pT1b der sonographisch falsch-negativen Befunde in absoluten Zahlen (US-BI-RADS 1-3)

Innerhalb der Patienten mit auffälligem sonographischen Befund zeigte die detaillierte Aufteilung der histopathologischen Befunde jeweils einen sehr hohen Anteil an der invasiven pT1b-Gruppe (56\%). Die invasive pT1a-Gruppe war mit 29\%, das DCIS mit $27 \%$ vertreten (Abb.4.2e).

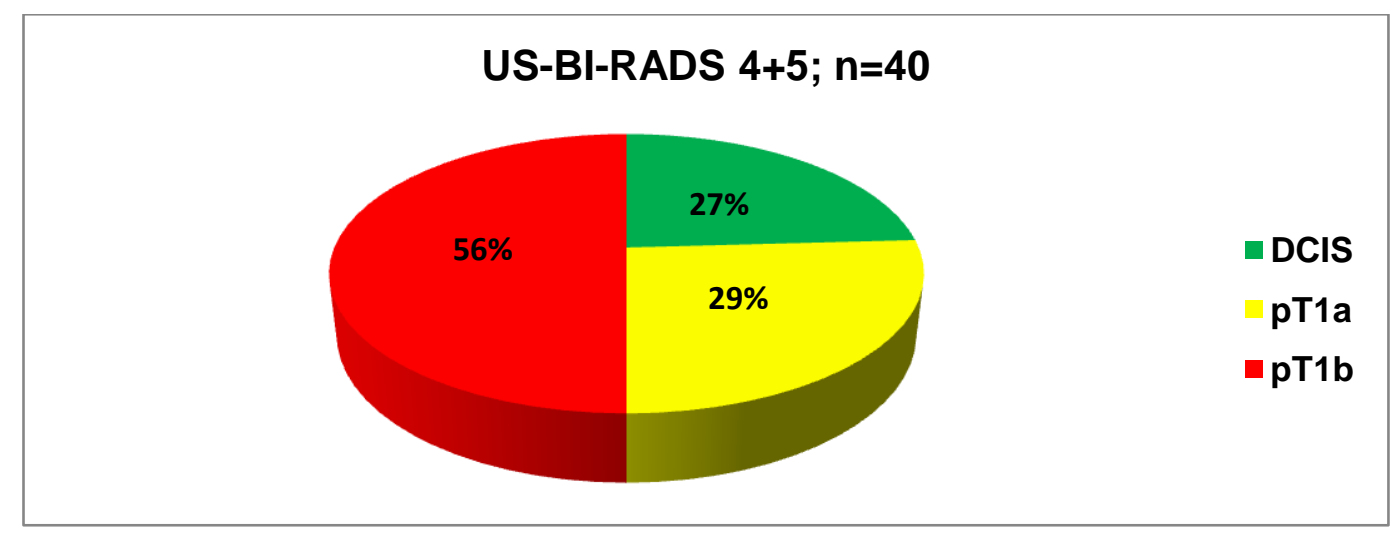

Abb.4.3e: Verteilung von DCIS, pT1a und pT1b in der Kategorie US-BIRADS 4 und 5 


\subsection{Ergebnisse Mamma-MRT}

Bei der Auswertung der Artefaktstufen dominierte die Artefaktstufe I mit 93\%. Geringe Bewegungs-und Subtraktionsartefakte (Artefaktstufe II) lagen in 6\% vor. Der Anteil grob störender Bewegungsartefakte lag bei 1\% (Abb.4.4a).

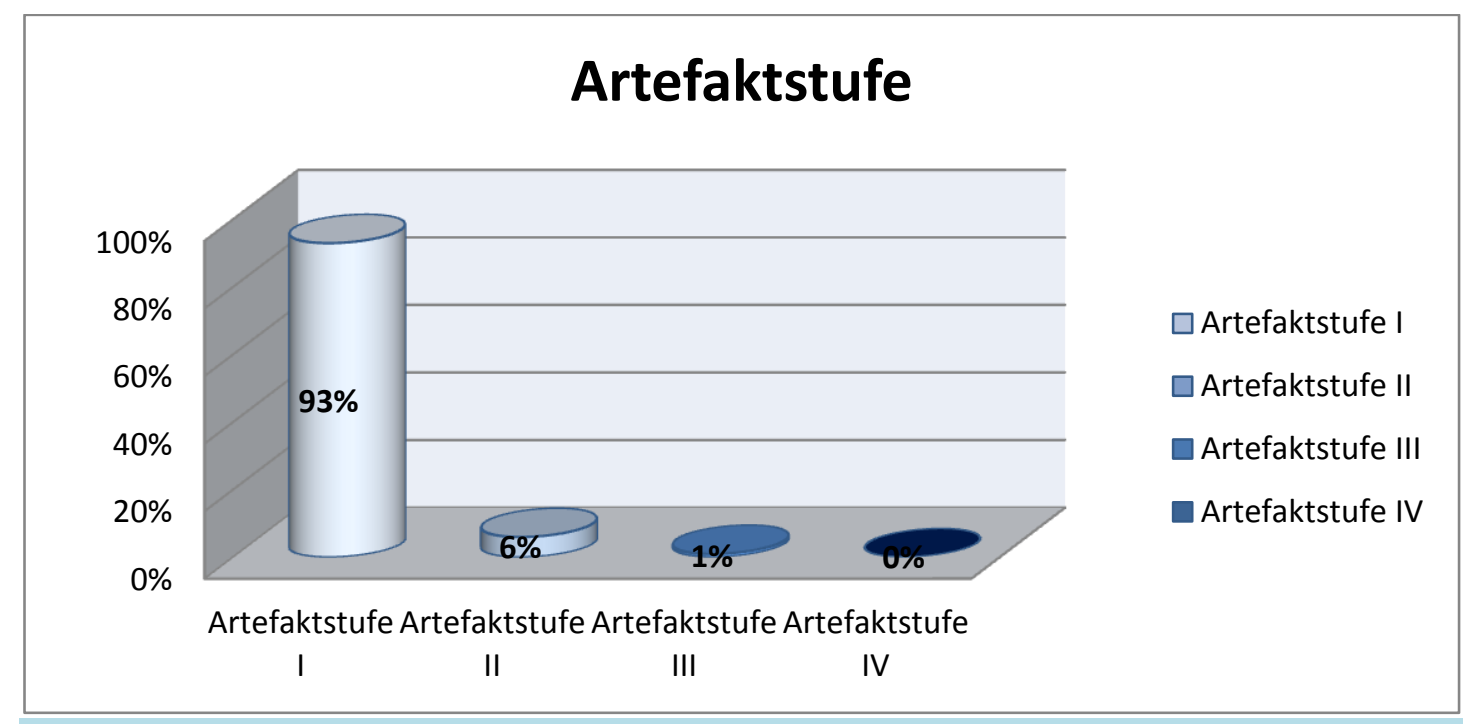

Abb.4.4a: Verteilung der Artefaktstufen I-IV in der Mamma-MRT

Hinsichtlich der „Dichte“ der Mamma-MRT ergab sich ein Anteil von 65\% für den Dichtetyp I mit keiner bis diskreter Kontrastmittelaufnahme des Parenchyms. In 24\% zeigte sich ein geringes, üblicherweise feinfleckiges Enhancement der parenchymalen Strukturen mit dem Dichtetyp II. MRM-Dichtetyp III und IV fanden sich zusammen in 11\% der Fälle (Abb.4.4b). 


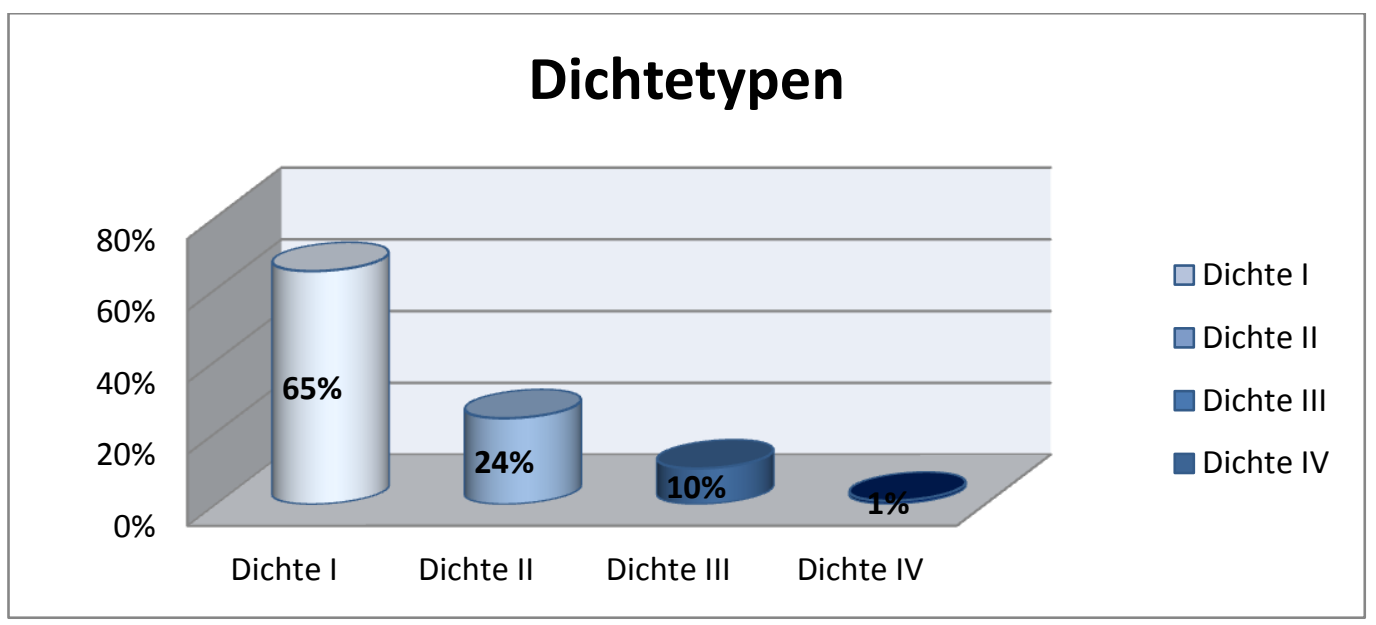

Abb.4.4b: Anzahl der Dichtetypen in der MR-Mammographie

Hinweise auf das Vorliegen eines bösartigen Mammakarzinoms fanden sich in der MR-Mammographie in $93 \%$ der Fälle. Sie ließ sich aufschlüsseln in die Kategorien MR-BI-RADS 4 (56\%) und MR-BI-RADS 5 (37\%). Die Befundgruppen MR-BI-RADS 1-3 mit unauffälliger bis wahrscheinlich benigner Befundung waren prozentual gering vertreten (Abb.4.4c).

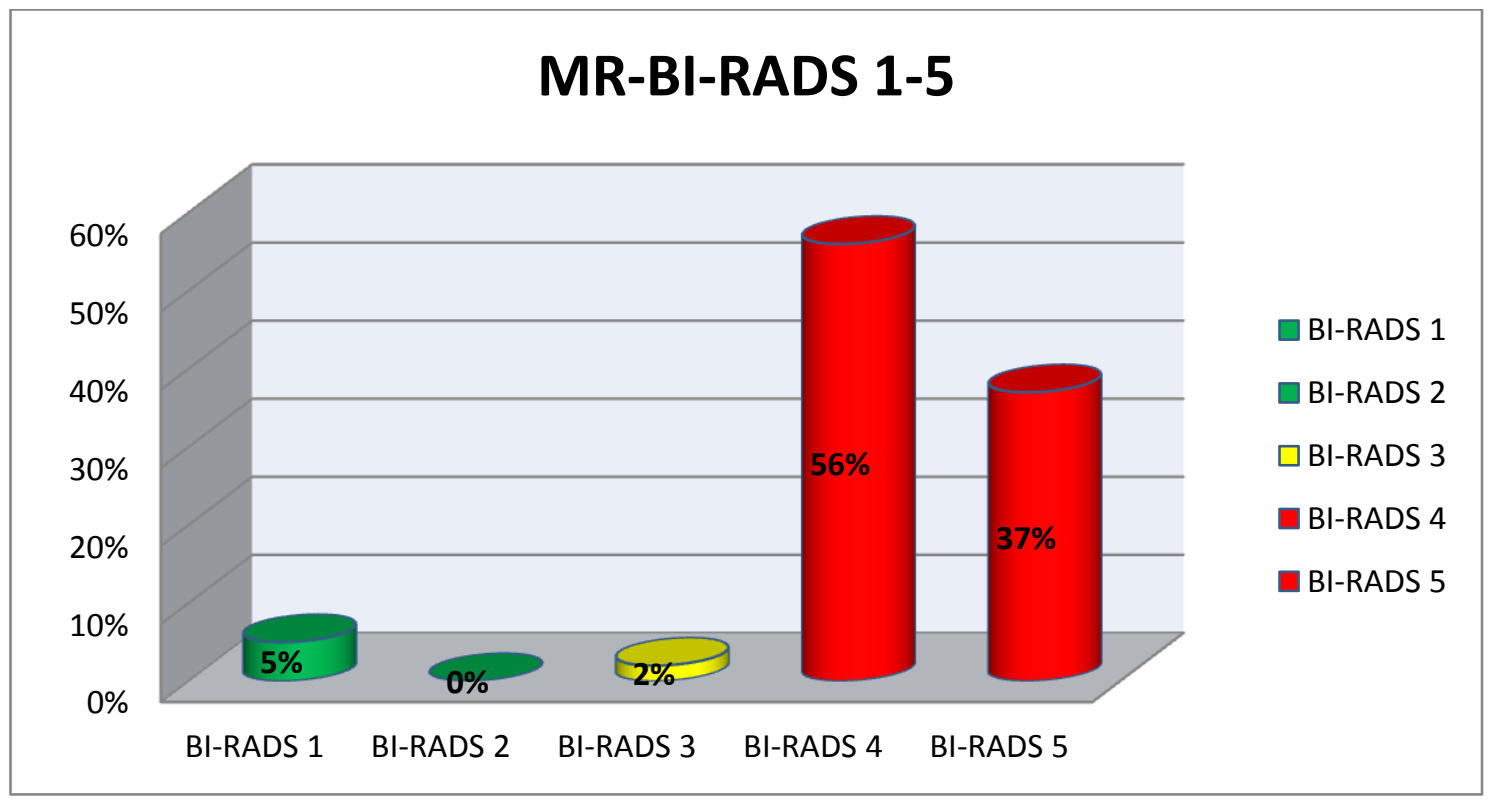

Abb.4.4c: Kategorisierung magnetresonanztomographischer Befunde bei 109 Patientinnen mit Mammakarzinom im Frühstadium nach MR-BI-RADS 1-5 
Innerhalb der Patientinnen mit auffälligem Befund zeigte die MR-mammographisch detaillierte Aufteilung der histopathologischen Befunde jeweils einen Anteil von 100\% in der invasiven Tumorgruppe pT1a bzw. pT1b-Gruppe. Das DCIS war in $84 \%$ vertreten (Abb.4.4d).

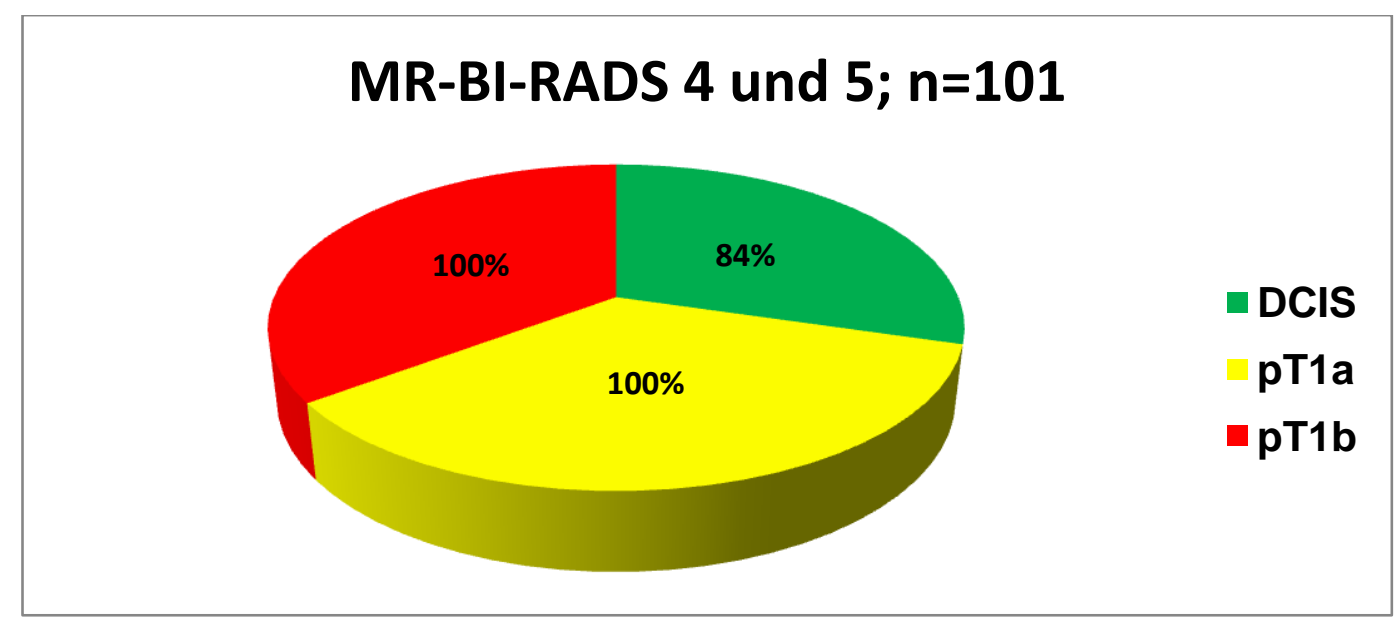

Abb.4.4d: Verteilung von DCIS, pT1a und pT1b in der Kategorie MR-BI-RADS 4 und 5

Für alle früh erkannten Tumoren zeigt Abb.4.4e die Verteilung des jeweiligen Hauptbefundes (Fokus, Herd, Non-mass-like-lesion) in der Mamma-MRT. Foki konnten nur in $14 \%$ in der DCIS-Gruppe und in ca. $5 \%$ in der Gruppe der invasiven Karzinome ermittelt werden. Herde fanden sich in $49 \%$ vom DCIS zum pT1a (44\%) bis hin zum pT1b-Stadium (79\%). In der DCIS-Gruppe steigerte sich der prozentuale Anteil an Herdbefunden von 11\% in der G1-Gruppe auf 14\% in der G2-Gruppe und $24 \%$ in der G3-Gruppe. In der low-grade-DCIS-Gruppe dominierten die „Non-masslike-lesions" in $89 \%$. In ca. $70 \%$ fanden sie sich in der G2 und G3-Gruppe. Sie nahmen anteilmäßig von $50 \%$ in der pT1a-Gruppe auf $16 \%$ in der pT1b-Gruppe ab. 


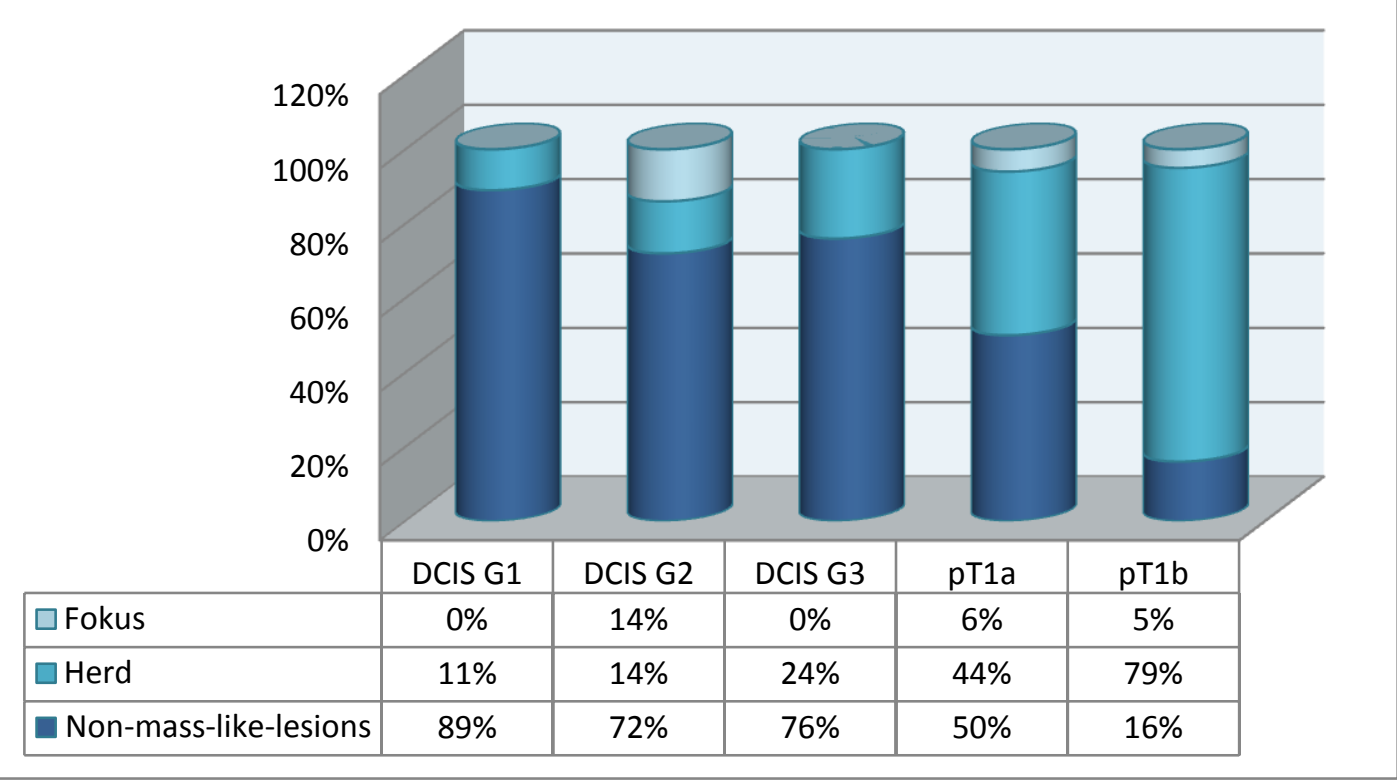

Abb.4.4e: Verteilung der DCIS-Gruppe G1-3, pT1a- und pT1b-Gruppe hinsichtlich der Anzahl an Fokus, Herd und Non-mass-like-lesion in der MR-Mammographie

Abb.4.4f beschreibt die Beziehung der falsch-negativen bzw. falsch-positiven Befunde innerhalb der verschiedenen MR-mammographischen Dichteklassen ACR IIV. Auffallend war die systematische Abnahme der richtig-positiven Befunde mit Zunahme des Dichtegrades. In der ACR -Gruppe IV wurde nur 1 Fall richtig-positiv eruiert, so dass dies eine Rate von $100 \%$ ergab.

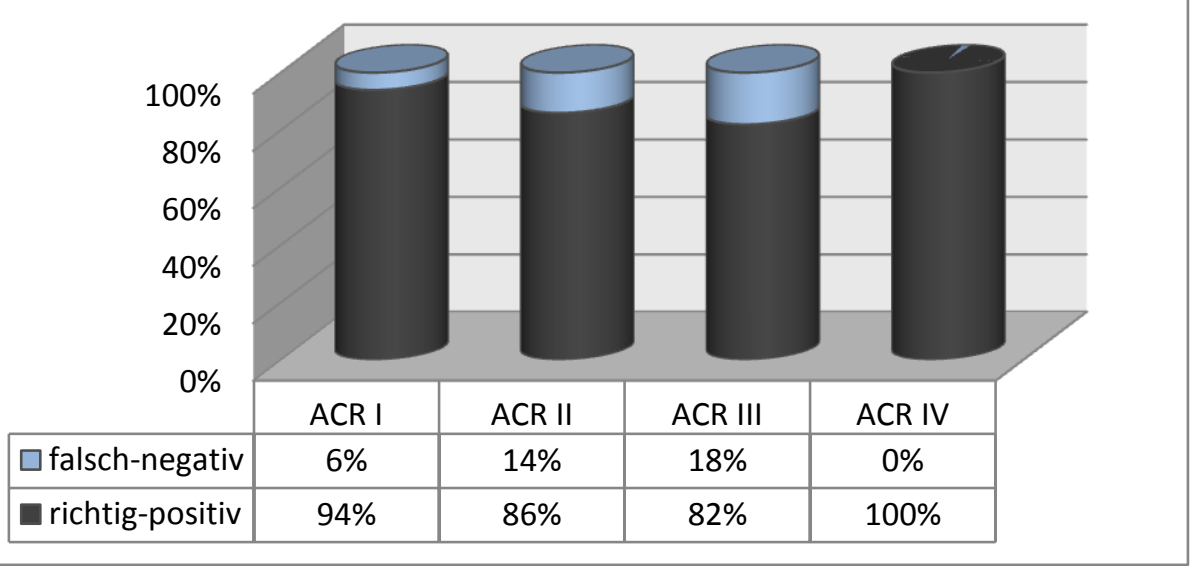

Abb.4.4f: Prozentuale Verteilung der falsch-negativen bzw. richtig-positiven Befunde innerhalb der verschiedenen MR-mammographischen Dichteklassen ACR I-IV 
In der Abb.4.4g wird abschließend deutlich, dass nur insgesamt 8 (7\%) von 109 Tumoren durch die MR-Mammographie nicht erkannt wurden. Es handelte sich bei diesem Kollektiv ausschließlich um DCIS-Tumore (16\% aller DCIS). Alle invasiven Tumore wurden eindeutig erkannt. Rechnet man bei dieser Auswertung die MR-BIRADS-Kategorie 3 hinzu, erhöht sich die Anzahl auf 10 (9\%), wobei die hinzukommenden 2 Fälle der high-grade-DCIS-Kategorie zugeordnet wurden.

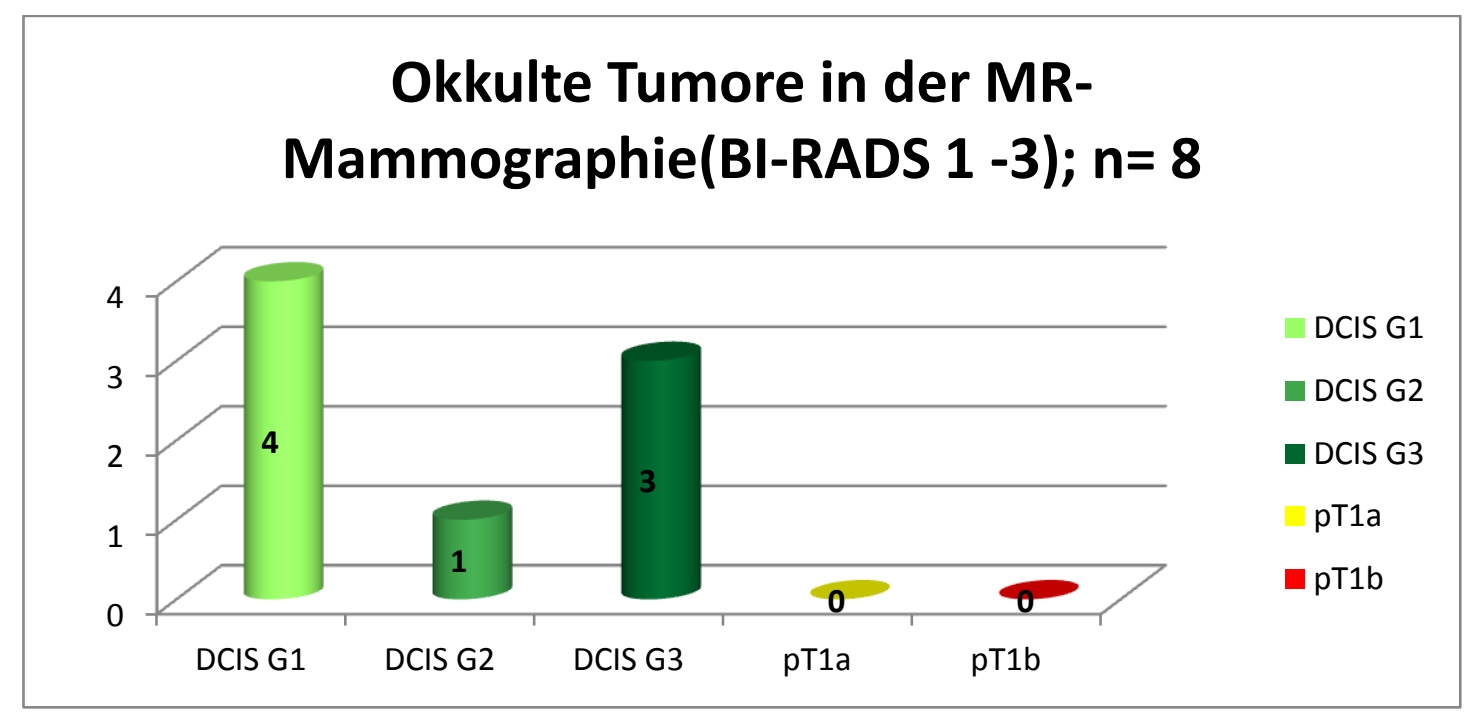

Abb.4.4g: Übersehene (okkulte) Tumore (falsch-negative Befunde) in der MR-Mammographie in absoluten Zahlen 


\subsection{Perkutane Biopsie}

Im ausgewerteten Kollektiv wurde überwiegend die MR-gesteuerte Vakuumbiopsie zur Abklärung von Befunden der Kategorien BI-RADS 4 oder 5 eingesetzt. In 30\% erfolgten eine ultraschallgesteuerte Stanze und mammographisch gesteuerte (stereotaktische) Biopsien in 25\%. Bei insgesamt 6\% des Kollektivs wurde keine diagnostische Exzision vorgenommen (DE) (Abb.4.5.1a).

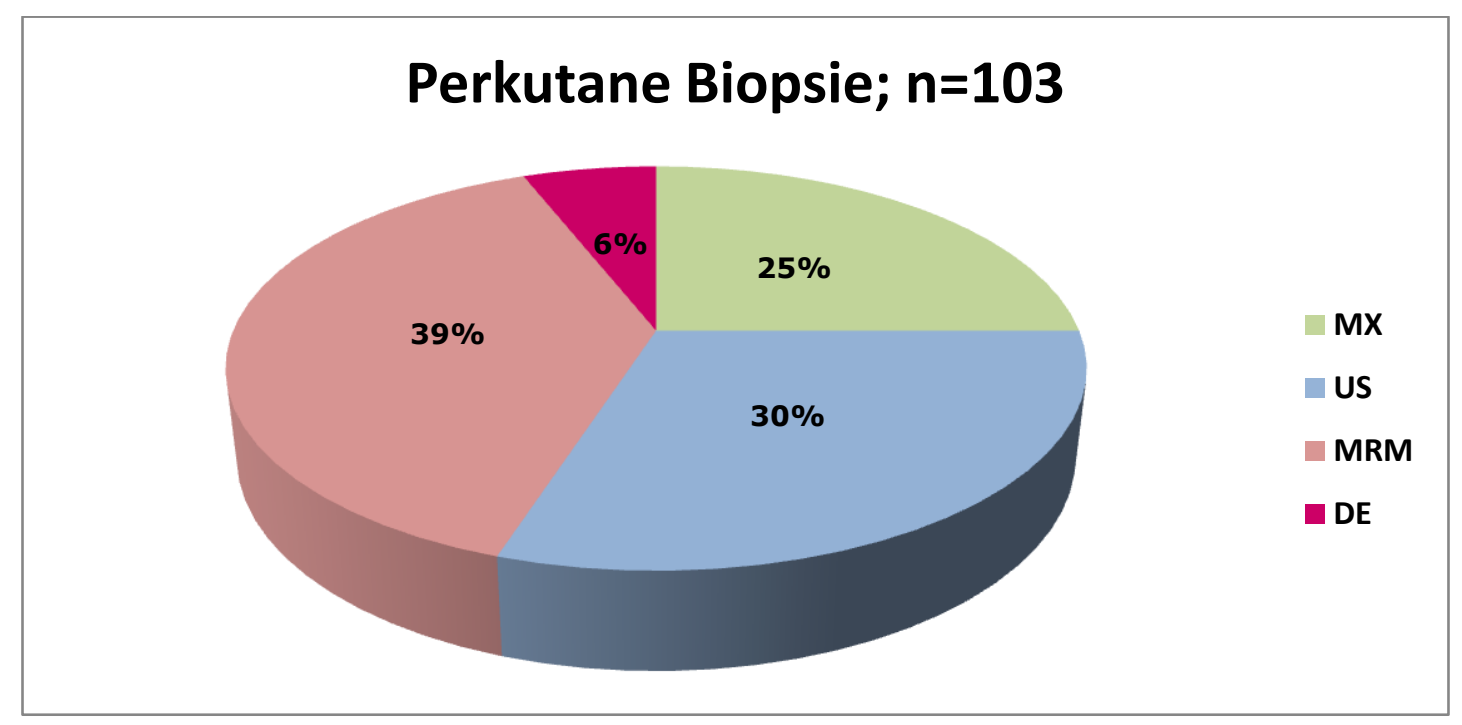

Abb.4.5.1a: Anzahl und führende Methode für die perkutanen Biopsie-Verfahren (Mammographie (MX), Ultraschall (US), MR-Mammographie (MRM), keine diagnostische Exzision (DE)) 


\subsection{Ergebnisse aller bildgebenden Verfahren}

Abb.4.6.1a zeigt die sicher erkannten Anteile an DCIS, pT1a und pT1b in den BIRADS-Kategorisierung 4 und 5 jeweils für die Mammographie, Sonographie und MRMammographie in der Übersicht. Den größten erkannten DCIS-Anteil konnte man in der MR-Mammographie mit $84 \%$ erkennen, in absteigender Reihenfolge mit $74 \%$ in der Mammographie und letztendlich mit $27 \%$ in der Ultraschallgruppe. Die invasive Gruppe wurde in $100 \%$ durch die MR-Mammographie detektiert. Die pT1a-Gruppe wurde in 53\% durch die Mammographie und in 29\% durch den Ultraschall, die pT1bGruppe wurde durch die Mammographie in 45\% und durch die Sonographie in 56\% korrekt diagnostiziert.

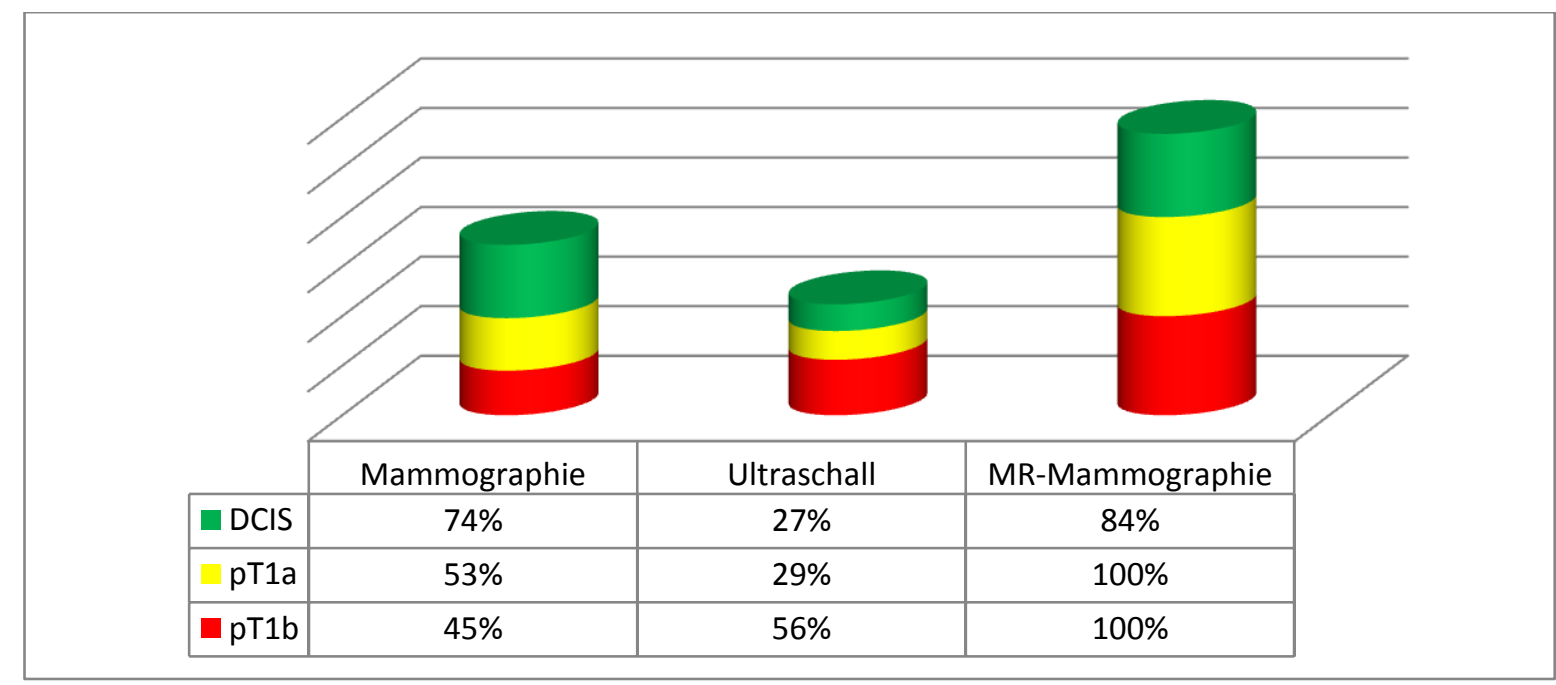

4.6.1a: Anteil richtig-positiver Befunde von DCIS, pT1a, pT1b in den BI-RADS-Gruppen 4 und 5 in der Mammographie, Sonographie und Mamma-MRT (DCIS: $n=50 ; p T 1 a: n=17 ; p T 1 b: n=42$ )

Abb.4.6.1b zeigt in der Übersicht den falsch-negativen Anteil von DCIS, pT1a, pT1b in den BI-RADS-Gruppen 1 und 2 (Mammographie (MX), Sonographie (US) und MRMammographie (MRM)). 


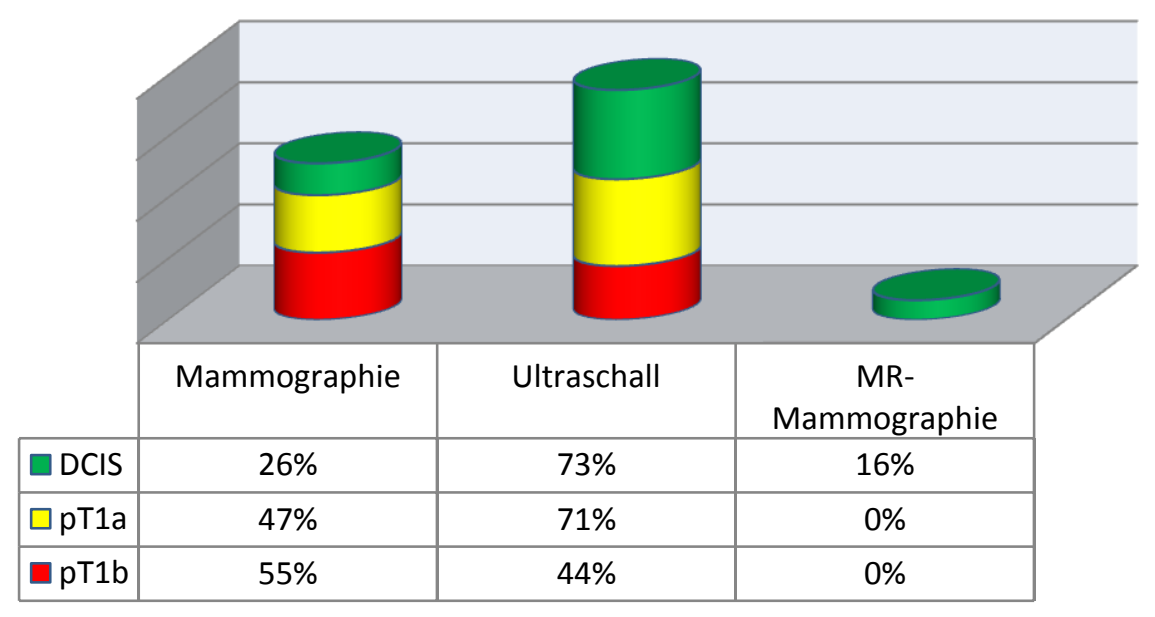

Abb.4.6.1b: Übersicht der falsch-negativen Befunde von DCIS, pT1a, pT1b in den BI-RADSGruppen 1-3 (Mammographie (MX), Sonographie (US) und MR-Mammographie (MRM); (DCIS: $n=50 ; p T 1 a: n=17 ; p T 1 b: n=42)$

\subsubsection{Kombination von Mammographie und Mamma-MRT}

In Abb.4.6.1c wird die Anzahl an falsch-negativen Befunden innerhalb der DCISTumoren bei Einsatz von Mammographie (MX) oder MR-Mammographie (MRT) innerhalb der BI-RADS-Kategorien 1 und 2 in absoluten Zahlen dargestellt. In Abb. 4.6.1d erfolgt die prozentuale Darstellung mit den gleichen Daten. Hier wurde insgesamt die höhere falsch-negative Rate der Mammographie im Vergleich zur MRMammographie deutlich.

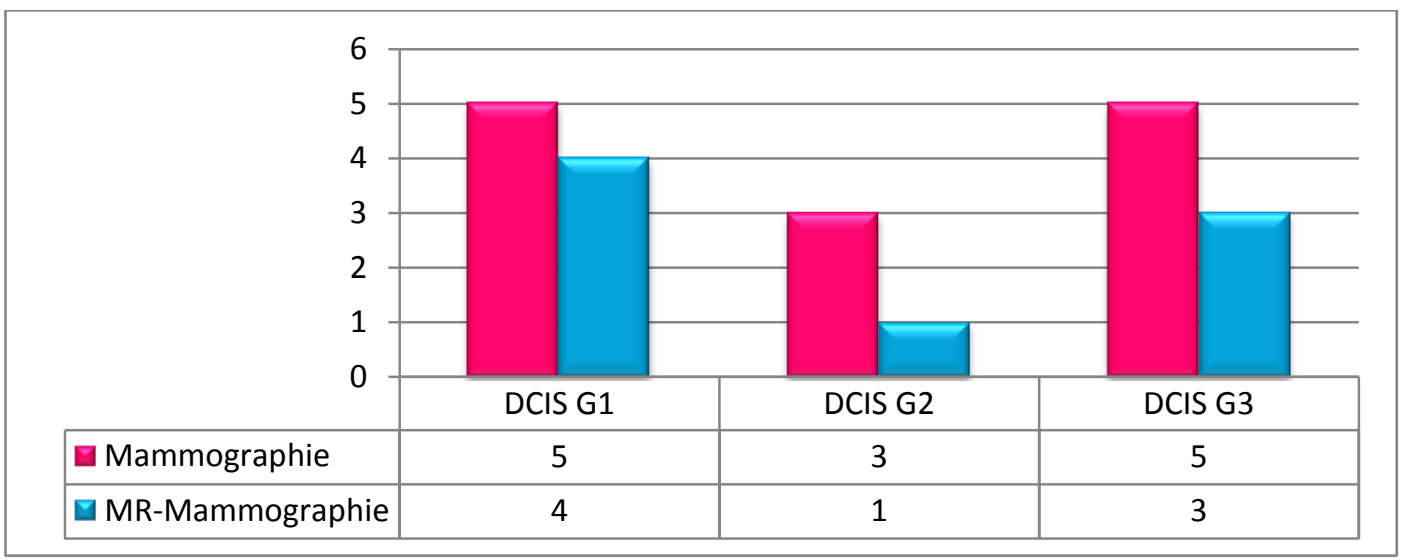

Abb.4.6.1c: Anzahl der falsch-negativen Befunde innerhalb der DCIS-Tumoren bei Einsatz von Mammographie (MX) und MR-Mammographie (MRT), (BI-RADS 1 und 2) in absoluten Zahlen (DCIS G1: $n=13$; DCIS G2: $n=14$; DCIS G3: $n=23$ ). 
Abb.4.6.1d und Abb.4.6.1e zeigen die Anteile der falsch-negativen Befunde in der Gruppe pT1a bzw. pT1b (BI-RADS 1 und 2). Es wurde deutlich, dass mammographisch $47 \%$ in der pT1a-Gruppe bzw. $55 \%$ in der pT1b-Gruppe falschnegativ befundet wurden. MR-mammographisch wurde in beiden Gruppen kein falsch-negativer Befund erhoben.

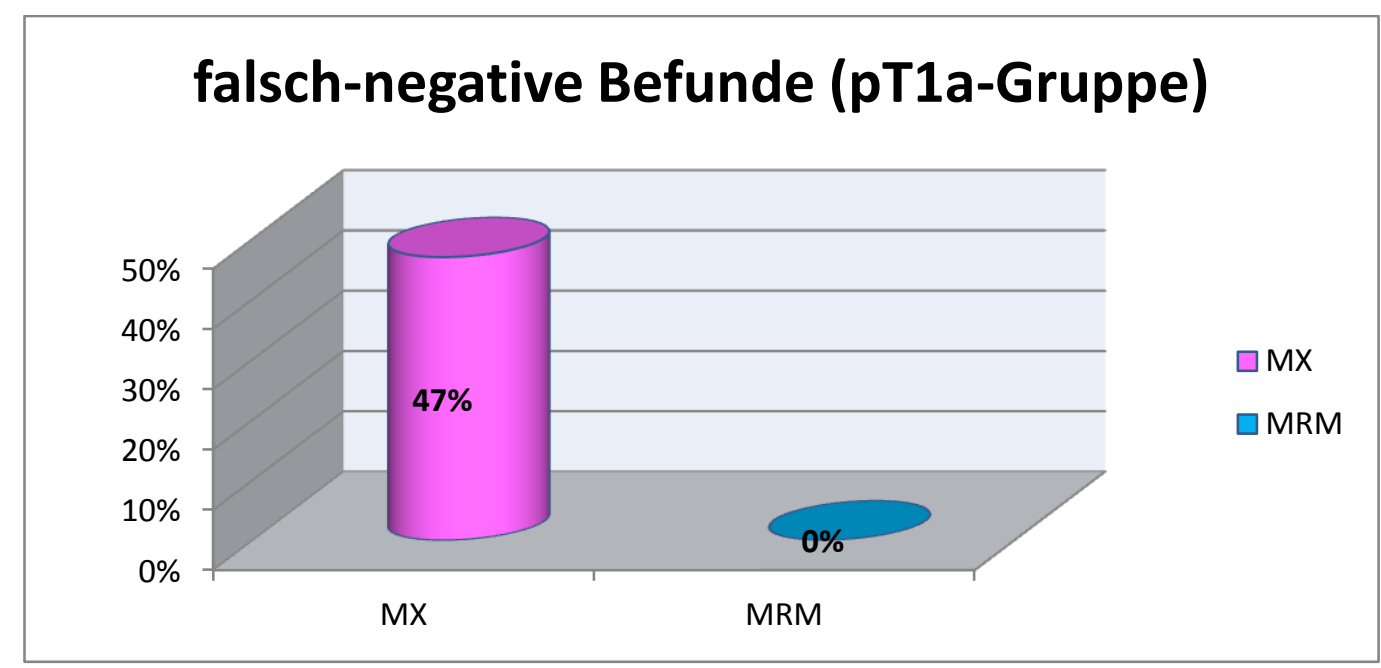

Abb.4.6.1d: Prozentualer Anteil der falsch-negativen Befunde innerhalb der pT1a-Gruppe von Mammographie (MX) und MR-Mammographie (MRM) (BI-RADS 1 und 2)

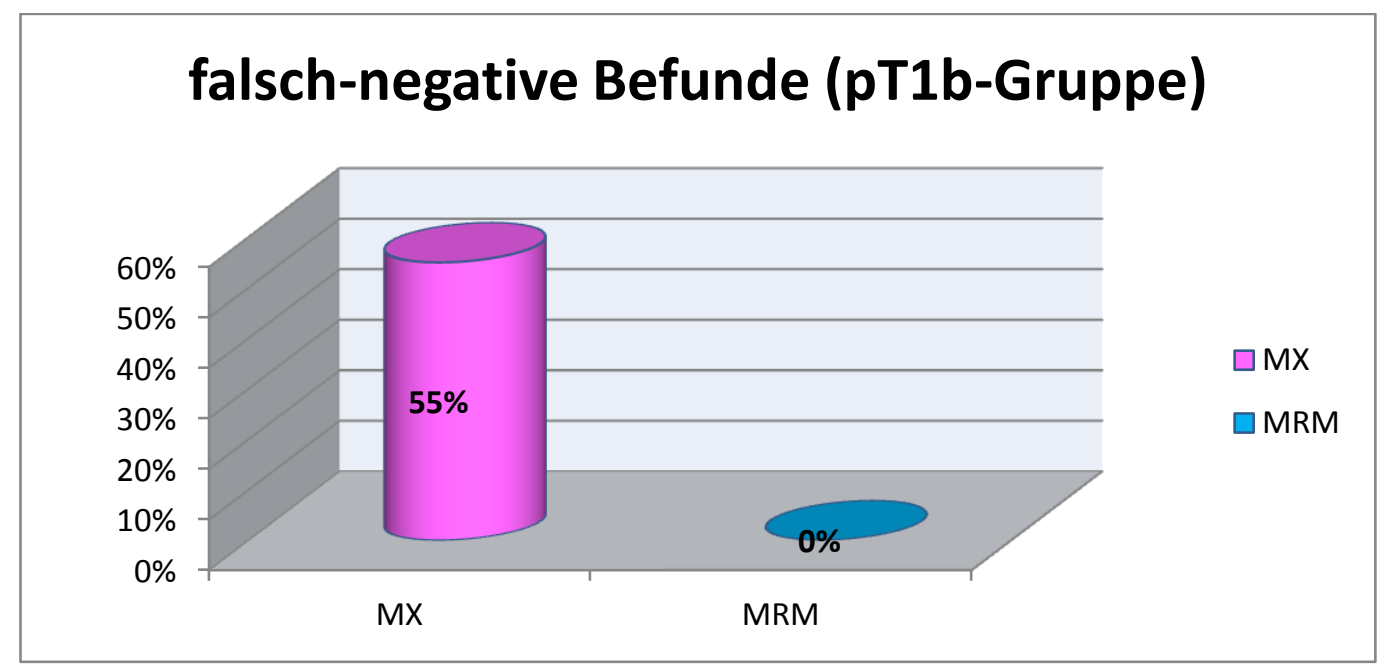

Abb.4.6.1e: Prozentualer Anteil der falsch-negativen Befunde innerhalb der pT1b-Gruppe von Mammographie (MX) und MR-Mammographie (MRM) (BI-RADS 1 und 2) 
Abb. 4.6.1f zeigt den mammographischen und MR-mammographischen Anteil der falsch-negativen Befunde aller invasiven Frühkarzinome. Insgesamt 52\% aller Frühkarzinome wurden mammographisch falsch-negativ zugeordnet und kein MRmammographischer Befund.

\section{falsch-negative Befunde der invasiven Frühkarzinome (pT1a und pT1b-Gruppe)}

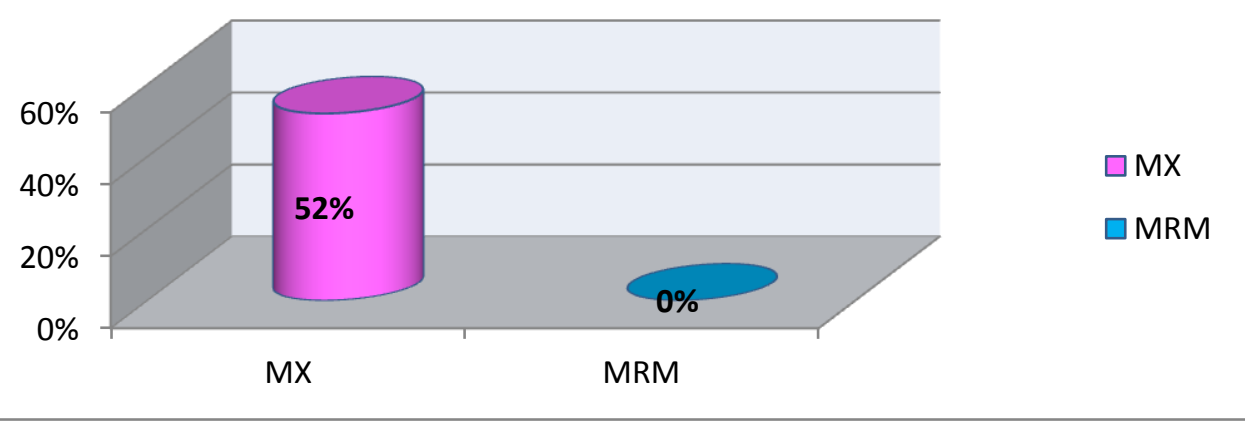

Abb.4.6.1f: Prozentualer Anteil der falsch-negativen Befunde innerhalb der invasiven Frühkarzinome (pT1a und pT1b) von Mammographie (MX) und MR-Mammographie (MRM) (BIRADS 1 und 2)

Abb.4.6.1g zeigt in der Übersicht die Verteilung der BIRADS-Kategorien 1-5 von Ultraschall (US), Mammographie (Mx) und Mamma-MRT (MRM) für das Gesamtkollektiv der früh erkannten Karzinome (DCIS, pT1a, pT1b). Für den Ultraschall ergab sich eine Quote von richtig-positiven Befunden von 39\%, für die Mammographie von $60 \%$ und für die Mamma-MRT von $93 \%$. Auch bei Berücksichtigung der Kategorie BI-RADS 3 als ,richtig-positiv“ ergaben sich keine signifikanten Veränderungen dieser Dimensionen. 


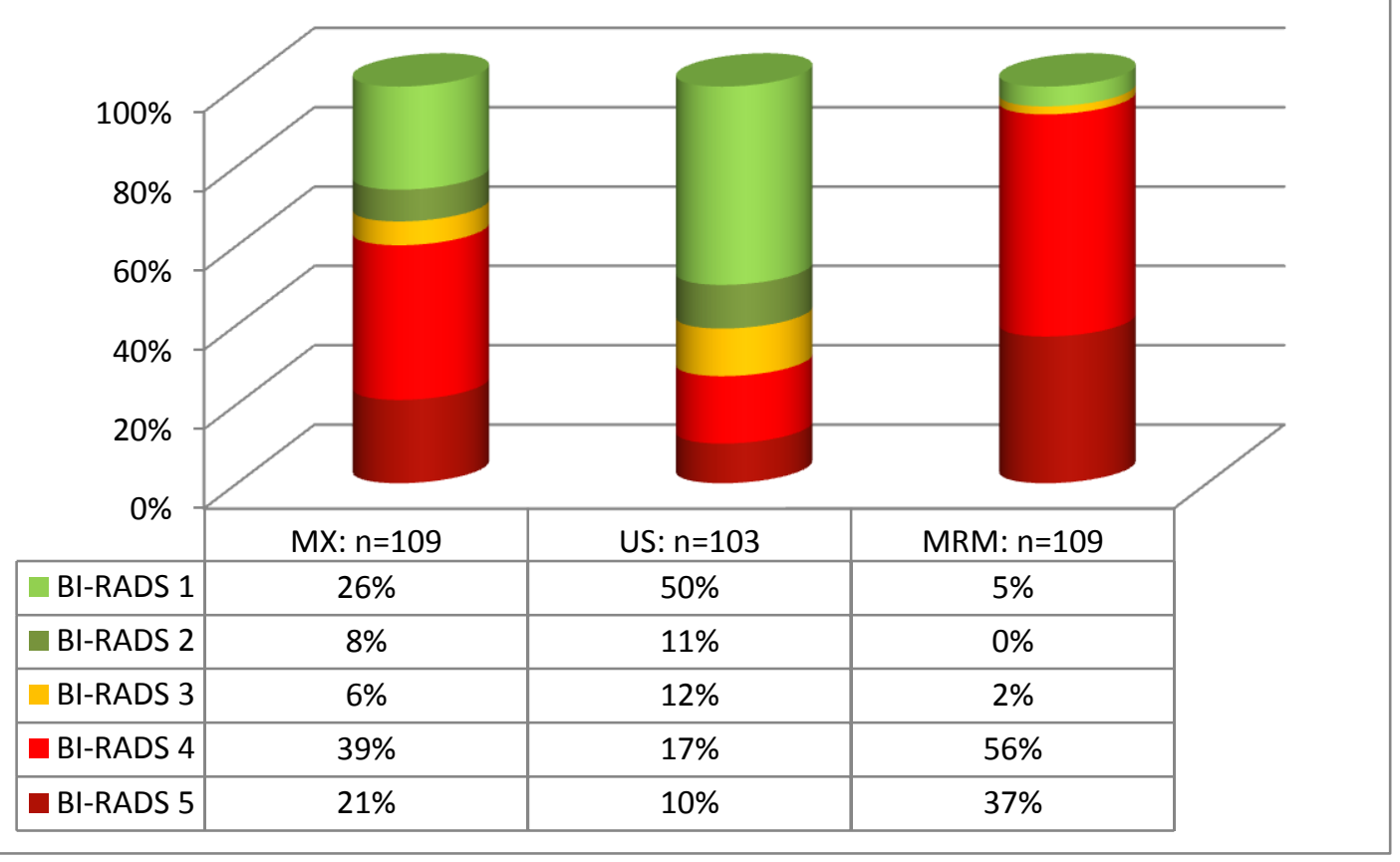

Abb. 4.6.1g: Prozentuale Verteilung der BI-RADS-Kategorien 1-5 von Mammographie (MX), Ultraschall (US) und MR-Mammographie (MRM) für alle früh erkannten Mammakarzinome (MX und MRM: $n=109$; US: $n=103$ ) 


\subsubsection{Kombination der verschiedenen Methoden und Tumordetektion}

Die Übersichtstabelle (Abb.4.6.2a) gibt Auskunft über die verifizierten Karzinome (DCIS G1, DCIS G2, DCIS G3, pT1a und pT1b) in der BI-RADS-Kategorie 4 und 5. Hier wurde die Überlegenheit der MR-Mammographie deutlich, da diese Methode die höchste Trefferquote in allen Kategorien aufzeigte.

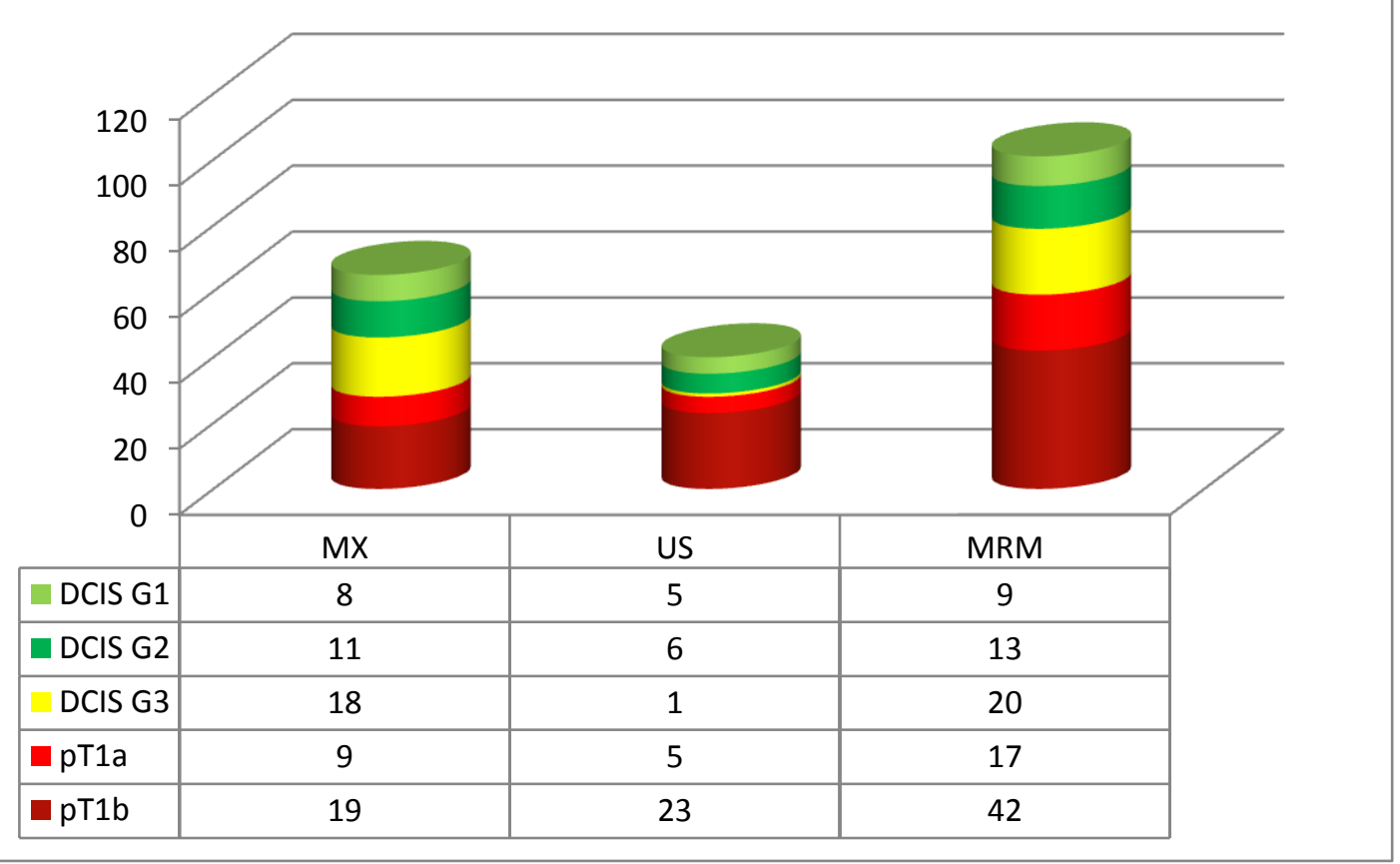

Abb.4.6.2a: Übersicht über die gefundenen Karzinome (DCIS G1, DCIS G2, DCIS G3, pT1a, pT1b) in der BI-RADS-Kategorie 4 und 5 in absoluten Zahlen (Mammographie (MX), Ultraschall (US), MR-Mammographie (MRM)) 


\subsubsection{Kombination von Mammographie und Ultraschall für DCIS, pT1a und pT1b und für alle Frühkarzinome}

Die Mammographie zeigte in der präsentierten Untersuchung in der Detektion von DCIS-Läsionen ( $n=50)$ eine Sensitivität von $74 \%(M X=37$ Fälle), der Ultraschall 27\% (US=12 Fälle).

In der Gruppe der pT1a-Tumoren $(n=17)$ konnten diese mammographisch mit 53\% $(\mathrm{MX}=9)$ detektiert werden, durch den Ultraschall mit 29\% (US=5 Fälle).

In der Gruppe der pT1b-Läsionen (n=42 Fälle) wurden 45\% (MX=19 Fälle) der Tumore mammographisch und 56\% (US=23 Fälle) sonographisch entdeckt.

Insgesamt $60 \% \quad(\mathrm{MX}=65 \quad$ Fälle $)$ aller Frühkarzinome $(\mathrm{n}=109)$ wurden mammographisch und 39\% (US=40 Fälle) sonographisch $(n=103)$ erkannt.

Insgesamt wurden 5 invasive pT1b-Tumore der mammographisch okkulten Tumore durch den Ultraschall erkannt.

\subsubsection{Kombination von MR-Mammographie und Ultraschall für DCIS, pT1a und pT1b und für alle Frühkarzinome}

Die MR-Mammographie zeigte in der präsentierten Untersuchung in der Detektion von DCIS-Läsionen $(n=50)$ eine Sensitivität von 84\% (MRM=42 Fälle), der Ultraschall 27\% (US=12 Fälle).

In der Gruppe der pT1a-Tumoren ( $n=17)$ konnten diese MR-mammographisch mit $100 \%(\mathrm{MRM}=17)$ detektiert werden, durch den Ultraschall mit 29\% (US=5 Fälle).

In der Gruppe der pT1b-Läsionen (n=42 Fälle) wurden 100\% (MRM=42 Fälle) der Tumore MR-mammographisch und 56\% (US=23 Fälle) sonographisch entdeckt.

Insgesamt 93\% (MRM=101 Fälle) aller Frühkarzinome $(n=109)$ wurden MRmammographisch und 39\% (US=40 Fälle) sonographisch $(n=103)$ erkannt.

Die Auswertung ergab, dass nur 1 DCIS (G3) von den 8 durch die Mamma-MRT nicht erkannten DCIS durch den Ultraschall erkannt wurde. 


\subsubsection{Kombination von Mammographie und MR-Mammographie für DCIS, pT1a und pT1b und für alle Frühkarzinome}

Die MR-Mammographie zeigte hinsichtlich der Detektion von allen DCIS-Läsionen $(\mathrm{n}=50)$ eine Trefferquote von $84 \%$ (MRM=42 Fälle). Die Mammographie detektierte $74 \%$ (MX=37 Fälle). Durch die MR-Mammographie wurde der größte Anteil in der high-grade-DCIS-Gruppe mit 20 Patienten gefunden, die Mammographie detektierte hier 18 Frauen. Auch in der DCIS-Gruppe 2 zeigte sich bei der MR-Mammographie eine Anzahl von 13 Frauen, bei der Mammographie 11 Frauen in dieser Gruppe. Auch in der low-grade-DCIS-Gruppe waren es annähernd gleiche Fallzahlen, bei der MR-Mammographie mit 9 und 8 Patientinnen bei der Mammographie (Abb. 4.6.2.3a und Abb. 4.6.2.3b).

In der pT1a-Gruppe ( $\mathrm{n}=17$ Fälle) zeigte sich die MR-Mammographie insgesamt mit 100\% (MRM=17 Fälle) führend. Die Mammographie detektierte 53\% (MX=9 Fälle).

In der pT1b-Gruppe ( $\mathrm{n}=42)$ zeigte sich die MR-Mammographie insgesamt mit $100 \%$ (MRM=42 Fälle) ebenfalls führend. Die Mammographie detektierte 45\% (MX=19 Fälle) (Abb. 4.6.2.3a und Abb. 4.6.2.3b).

Für alle Frühkarzinome zusammen entdeckte die MR-Mammographie 93\% (101 Fälle), die Mammographie 60\% (65 Fälle).

Abb. 4.6.2.3a zeigt die absolute Anzahl der richtig-positiven Befunde innerhalb der DCIS-, pT1a- bzw. pT1b-Gruppe.

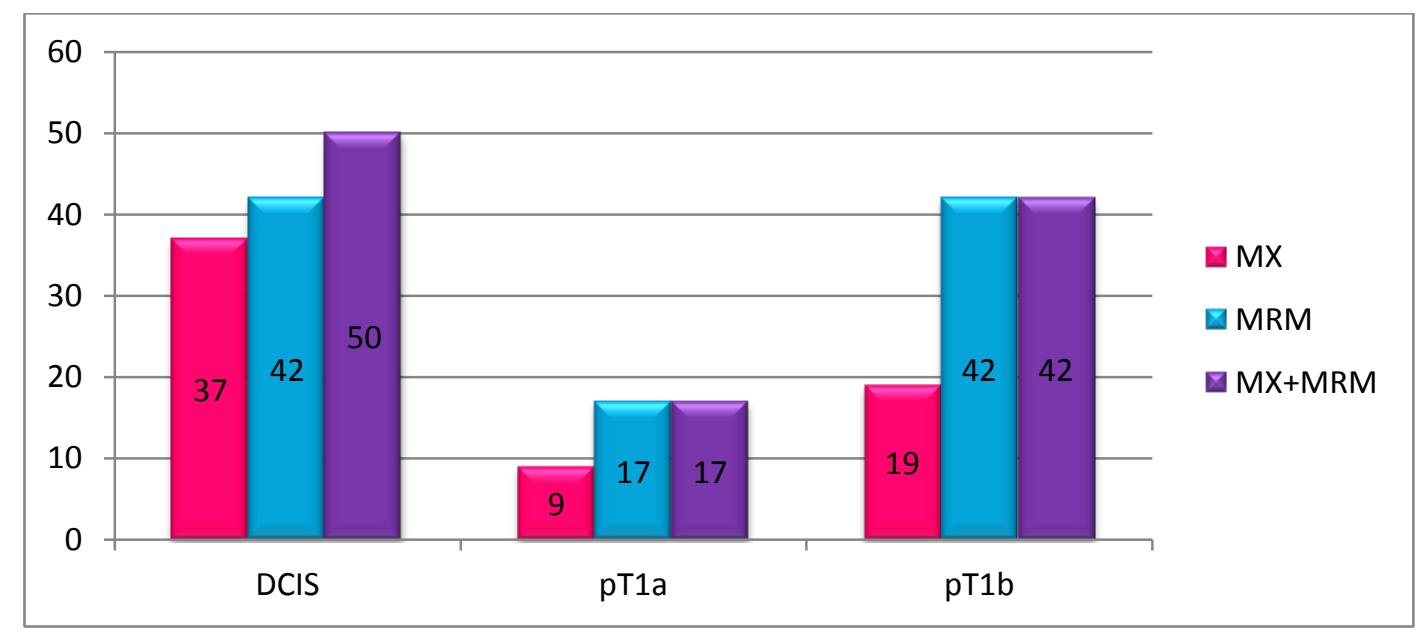

Abb.4.6.2.3a: Übersicht der richtig-positiven Befunde von DCIS, pT1a und pT1b in der Mammographie (MX), MR-Mammographie (MRM) und Mammographie und MR-Mammographie (MX+MRM) in absoluten Zahlen 
Abb.4.6.2.3b zeigt die absolute Anzahl der richtig-positiven Befunde innerhalb der DCIS-Gruppe 1-3.

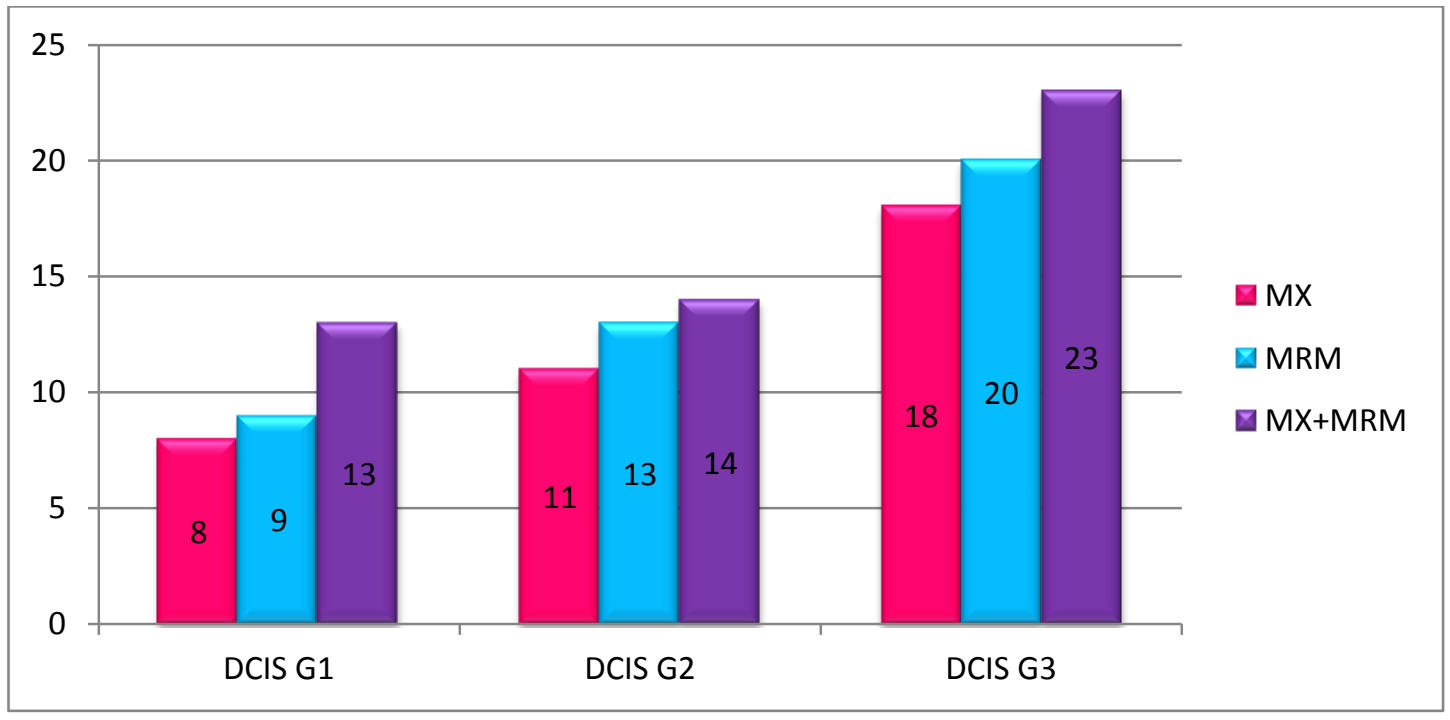

Abb.4.6.2.3b: Übersicht der richtig-positiven Befunde von DCIS 1-3 in der Mammographie (MX), MR-Mammographie (MRM), Mammographie und MR-Mammographie (MX+MRM) in absoluten Zahlen (DCIS G1:n=13; DCIS G2: n=14; DCIS G3: n=23; Gesamt-DCIS: $n=50$ )

Zusammenfassend zeigte die Mammographie über die Ergebnisse der Mamma-MRT hinaus zusätzlich 4 DCIS G1, 1 DCIS G2 und 3 DCIS G3. Für alle frühen invasiven Stadien (pT1a, pT1b) zeigte die Mammographie keinen Befund, der nicht auch in der MRT erkannt wurde. 


\section{Diskussion}

Brustkrebs ist ein heterogenes Krankheitsbild, dessen Verlauf durch unterschiedliche Entstehungsursachen, differentes Zellbild, variierende Tumorlokalisation und Tumorausdehnung bestimmt wird [S3-Leitlinie... Mammakarzinom 2008]. Die Früherkennung von Brustkrebs ist daher in Bezug auf die Diagnosestellung und Behandlung entscheidend, weil dadurch die Brustkrebssterblichkeit gesenkt und die Lebensqualität verbessert werden kann [Albert et al. 2009].

Durch Kenntnis der prognostischen Faktoren kann eine Voraussage über den individuellen Krankheitsverlauf und letztlich eine risikoadaptierte Therapie eingeleitet werden, um eine Verbesserung der individuellen Prognose zu erreichen.

\subsection{Brustkrebs im frühen Stadium}

Ein wesentlicher prognostischer Faktor ist das Tumorstadium zum Zeitpunkt der Diagnosestellung. Es ist bekannt, dass die Tumorgröße mit dem axillären Lymphknotenbefall korreliert [Carter et al. 1989]. Dies geschieht umgekehrt proportional mit der Überlebenszeit. Bei Therapiebeginn liegen im Stadium pT1a nur selten Lymphknotenmetastasen vor, während eine nodale Metastasierung im pT1cStadium in $24 \%$ der Fälle und im pT3/4-Stadium bereits in über $80 \%$ der Fälle nachzuweisen ist [Prechtel 1982]. Der Zusammenhang zwischen TNM-Stadium und Prognose macht deutlich, dass die Früherkennung von eminenter Bedeutung ist. Ziel ist es daher, intraduktale Tumorstadien und invasive Tumoren in einer Größe bis 1 $\mathrm{cm}$ frühzeitig zu detektieren, um eine mögliche Verbesserung der Heilungschancen zu erreichen und im Einzelfall eine weniger radikale Therapie einzuleiten. Auch nach den S3-Leitlinien von 2008 sollte die Erkennung der präinvasiven Form oder des frühen invasiven Stadiums angestrebt werden, da die 5-Jahres-Überlebensrate hier bei über 90\% liegt [Engel et al. 2002; S3-Leitlinie... Mammakarzinom 2008]. Der zunehmende Nachweis präinvasiver Befunde lässt dabei auf einen präventiven Beitrag zur Senkung der Inzidenz invasiver Karzinome hoffen [Ries et al. 2006].

\subsection{Therapeutisches Vorgehen bei präinvasiven bzw. früh-invasiven Tumoren}

Die primäre Therapie des Mammakarzinoms ist die chirurgische Sanierung, gefolgt von strahlentherapeutischen und chemotherapeutischen Maßnahmen.

Das DCIS stellt einen heterogene Tumorart dar, bei der die Entscheidung über eine brusterhaltende Maßnahme oder eine Komplettentfernung des Drüsenkörpers von verschiedenen Faktoren abhängt. 
Allgemein gilt, dass bei einer Tumorgröße von $<2,5 \mathrm{~cm}$ zu einer brusterhaltenden Therapie (BET) geraten wird, bei einer Tumorgröße von $>4,5 \mathrm{~cm}$ zu einer Mastektomie ohne weitere Therapiemaßnahmen [Mammakarzinom: Manual Diagnostik und Therapie 2005]. Eine BET ist grundsätzlich möglich, wenn freie Resektionsränder (mehr als $10 \mathrm{~mm}$ Sicherheitsabstand) vorliegen und eine günstige Tumor-Brustrelation bestehen sowie bei fehlendem Hinweis auf Multizentrizität oder Invasivität im histopathologischen Befund. Auf eine Lymphonodektomie kann verzichtet werden. Bei ausgedehnten Befunden kann eine Sentinel-Node-Biopsie indiziert sein, um den Nodalstatus zu bestimmen. Im Fall einer Lymphknotenmetastasierung ist von einem invasiven Tumorgeschehen auszugehen und es können dann weiterführende Maßnahmen eingeleitet werden (Axilladisskektion, axilläres Lymphknotensampling, Sentinel-Lymphknotenbiopsie) [Kreienberg et al. 2006].

Bei invasiven Tumoren wird ebenfalls unter den o.g. Kriterien zu einer BET im Sinne einer Tumorektomie, Segmentresektion oder Quadrantektomie geraten. Bei fortgeschrittener Tumorgröße, ausgedehnten EIC-Anteilen, Multizentrizität, beim inflammatorischen Mammakarzinom und bei Tumoreinbruch in umgebende Strukturen kommt eher eine Mastektomie in Betracht [Mammakarzinom: Manual Diagnostik und Therapie 2005].

Eine adjuvante Nachbestrahlung ist grundsätzlich nach einem brusterhaltenden operativen Vorgehen bei invasiven Karzinomen indiziert, um hiermit eine lokale Tumorkontrolle zu gewährleisten. Beim DCIS hängt diese Entscheidung von der Ausdehnung, dem Grading und anderen Faktoren ab. In einer randomisierten Studie von Fisher et al. konnte gezeigt werden, dass nach der Bestrahlung der Restbrust beim DCIS signifikant weniger Rezidive auftraten als bei einer Nichtbestrahlung [Fisher DE et al. 1993]. Die Rate an Rezidiven verringert sich innerhalb von 8-10 Jahren nach Bestrahlung auf 5-10\%, insbesondere bei Frauen unter 50 Jahren [Bartelink et al. 2001]. Silverstein zeigte, dass bei einem Van-Nuys-Prognostic-Index (VNPI) von 7-9 Punkten Patientinnen erheblich von einer Bestrahlung profitierten, während bei einem Index von 4-6 Punkten keine Vorteile hinsichtlich des Rezidivrisikos zu verzeichnen waren. Auffallend war, dass bei einem Index von 10-12 Punkten nur eine leichte Rezidivsenkungsrate erfolgte, so dass hier eine Mastektomie indiziert war [Silverstein 2003].

Indikationen für eine adjuvante postoperative Bestrahlung nach Mastektomie sind T3/T4-Tumoren, T2-Tumoren mit einer Größe von mehr als $3 \mathrm{~cm}$, lymphogene Metastasierung, Befall der Pektoralisfaszie sowie ein Sicherheitsabstand von $<5 \mathrm{~mm}$ [Mammakarzinom: Manual Diagnostik und Therapie 2005]. Das Gesamtüberleben wird durch eine adjuvante Strahlentherapie nicht beeinflusst, daher sollte die Indikation diesbezüglich bei steigendem Lebensalter kritisch betrachtet und sorgfältig hinsichtlich des Nebenwirkungsprofils abgewogen werden. 
Anthrazykline stellen den Grundbaustein der adjuvanten Chemotherapie dar. Der sogenannte "Oxford Overview", sowie die Metaanalyse der Early Breast Cancer Trialists 'Collaborative Group (EBCTCG) zeigte einen signifikanten Überlebensvorteil bei dem Einsatz anthrazyklinhaltige Mehrfachkombinationen im Vergleich zu CMF (Cyclophosphamid, Methotrexat und 5-Fluorouracil) [Chia et al. 2005]. Nachteilig wirkt sich aber auch z.B. die Kardiotoxizität eines antthrazyklinhaltigen Regimes aus [Henderson 2011]. Nach Henderson et al. werden taxanhaltige Schemata für die adjuvante Therapie bei Nodal-positiven Patientinnen uneingeschränkt und bei Nodalnegativen Patientinnen im Falle des Vorliegens zusätzlicher Risikofaktoren empfohlen. Bei Nodal-positiven und Rezeptor-negativen Patientinnen konnte hier ein günstiger Verlauf beschrieben werden [Henderson et al. 2001]. Nach Clarke sollte immer einige Monate eine Polychemotherapie verabreicht werden, da es eine Verbesserung von $7-11 \%$ in der 10-Jahres-Überlebensrate von Frauen unter 50 Jahren und 2-3\% bei Frauen zwischen 50-69 Jahren mit frühem Brustkrebs bewirkt [Clarke 2008]. In randomisierten Studien konnte nachgewiesen werden, dass Kombinationschemotherapeutika mit Taxanen und/ohne Anthrazykline genauso effektiv zu sein scheinen. Daher sollten Anthrazykline nur bei aggressiven Tumorformen und selektiertem Patientenkollektiv angewendet werden [Henderson 2011]. In einer randomisierten Studie erzielte die Monotherapie mit Paclitaxel gleiche Ergebnisse wie eine Kombinationschemotherapie [Possinger und Flath 1998].

Die Gabe einer adjuvanten antiöstrogenen Therapie mit Tamoxifen scheint beim Hormonrezeptor-positiven DCIS sinnvoll [Purushotham 2003]. Es gibt aber keinen Nachweis eines präventiven Nutzen bei Hochrisikopatientinnen [Virnig et al. 2009]. Für den Einsatz von Aromatasehemmern bei präinvasiven Tumoren liegen keine ausreichenden Daten vor.

Die Ausschaltung der Ovarialfunktion bei prämenopausalen Frauen durch $\mathrm{GnRH}$ Analoga oder Ovarektomie kann den Krankheitsverlauf günstig beeinflussen. Die Wirksamkeit ist vergleichbar mit einer CMF-Chemotherapie. Eine Therapie mit GnRH-Analoga sollte über mindestens 2 Jahre durchgeführt werden [S3-Leitlinie... Mammakarzinom 2008].

Der Aromatasehemmer Letrozol beeinflusst das krankheitsfreie Intervall bei postmenopausalen Frauen mit Rezeptor-positiven frühem Mammakarzinom positiv. Nach der BIG-1-98-Studie von 2009 konnte festgestellt werden, dass eine sequentielle Behandlung von Letrozol und Tamoxifen, verglichen mit der Monotherapie von Letrozol oder Tamoxifen, keine statistisch signifikante Verbesserung des krankheitsfreien Intervalls mit sich brachte [Mouridsen et al. 2009]. Die ATAC-Studie untersuchte die Wirksamkeit und Sicherheit einer fünfjährigen adjuvanten Behandlung mit Anastrozol oder Tamoxifen bei postmenopausalen Frauen mit frühem Mammakarzinom. Im Vergleich zu Tamoxifen sprechen die bisher publizierten 5-Jahresergebnisse für ein signifikant verlängertes krankheitsfreies Überleben, geringere Rezidivraten sowie weniger Fernmetastasen und kontralaterale Rezidive mit Anastrozol. 
Das krankheitsfreie Überleben war in der Gesamtpopulation und bei Hormonrezeptor-positiven Patientinnen mit Anastrozol signifikant besser als mit Tamoxifen. Keine Unterschiede bestanden beim Gesamtüberleben [Cuzick et al. 2010]. Patientinnen mit einem frühen, operablen und metastasierten Mammakarzinom und gleichzeitiger HER2/neu-Überexpression profitieren von der Gabe des monoklonalen Antikörpers Trastuzumab. Wenn dieser neoadjuvant und adjuvant mit einer entsprechenden neoadjuvanten Chemotherapie bei lokal fortgeschrittenem und/oder inflammatorischem Mammakarzinom kombiniert wird, kann das Gesamtüberleben positiv beeinflusst werden [Gianni et al. 2010].

\section{3 Überleben und Langzeitüberleben}

Nach unbehandeltem DCIS liegt das Risiko eines nachfolgenden invasiven Karzinoms bei 30-50 \% [Lebeau 2006; S3-Leitlinie... Mammakarzinom 2008]. Die Gesamtüberlebensrate nach adäquater Therapie ist exzellent und beträgt $96-98 \%$, allerdings steigt die Gefahr eines lokoregionären Rezidivs bei alleiniger operativer Sanierung. Ungefähr 50\% aller Rezidive sind invasive Läsionen [Khan und Newman 2004].

Die Überlebenszeit bei invasiven Karzinomen hängt vom Stadium des Tumors bei der Erstdiagnose ab. Von den Frauen mit Tumoren pT1 N0 M0 überlebten 99,0\% (relativ) die Zeitspanne von 5 Jahren. Diese Quote verschlechterte sich bei Frauen mit Tumoren pT2 N0 M0 auf 92,1\% und betrug für die Stadien pT3 N0 M0 79\% und für pT4 N0 M0 nur noch 73,8\%. Bei pT1 N0 M0 Befunden werden nach 15 Jahren brustkrebsbedingte Sterbefälle von $11,7 \%$ beobachtet. [www. tumorregistermuenchen.de/facts/specific-analysis.html]. Während bei invasiven Tumorgrößen unter $1 \mathrm{~cm}$ (pT1a, pT1b) zum Diagnosezeitpunkt keine Fernmetastasen vorlagen, betrug die entsprechende Rate bei fortgeschrittenen Tumoren bereits $18,2 \%$ [Schneider et al. 2003].

Die nachfolgende Tabelle aus zeigt den Einfluss der Tumorgröße auf das Langzeitüberleben bei Brustkrebs. Bei einer Tumorgröße unter $10 \mathrm{~mm}$ ist ein rezidivfreies Überleben von $88 \%$ möglich. Je größer der Tumor ist, desto mehr sinkt das rezidivfreie Überleben. Bei einer Primärgröße von 17-22 mm beträgt das rezidivfreie Überleben nur noch 59\% [Rosen und Groshen 1990] (Tab.5.3a). 


\begin{tabular}{l|l}
\hline Tumorgröße $(\mathrm{mm})$ & Rezidiv-freies Überleben \\
\hline$<10$ & $88 \%$ \\
$11-13$ & $73 \%$ \\
$14-16$ & $65 \%$ \\
$17-22$ & $59 \%$ \\
\hline
\end{tabular}

Tab.5.3a: Einfluss der Tumorgröße auf das Langzeitüberleben bei Brustkrebs [Rosen und Groshen 1990]

Während die relative Fünfjahresüberlebensrate bei Nodal-negativen Patientinnen 94,8\% beträgt, sinkt sie bei zehn oder mehr befallenen Lymphknoten auf 48,1\% [Engel et al. 2003]. Tabelle 5.3b vergleicht die Anzahl der befallenen Lymphknoten mit der 5-Jahres-Überlebensrate. Diese sinkt mit der Anzahl der befallenen Lymphknoten. Bei einem Befall von mehr als 13 Lymphknoten beträgt die 5-JahresÜberlebensrate hier nur noch $28,4 \%$ [Osborne 1990].

\begin{tabular}{l|l}
\hline Anzahl positiver Lymphknoten & 5-Jahres-Gesamtüberleben \\
\hline 0 & $82,8 \%$ \\
$1-3$ & $73 \%$ \\
$4-12$ & $45,7 \%$ \\
$>13$ & $28,4 \%$ \\
\hline
\end{tabular}

Tab.5.3b: Korrelation von Anzahl der befallenen Lymphknoten mit der 5-JahresGesamtüberlebensrate [Osborne 1990]

\subsection{Frühdiagnostik des Mammakarzinoms}

Die Selbstuntersuchung bzw. klinische Untersuchung stellt in der Früherkennung keine hinreichend effiziente Form dar, da nur durchschnittlich Tumoren in einer Größenordnung von 2-3 cm getastet werden können [Mammakarzinom: Manual Diagnostik und Therapie 2005]. Ratanachaikanont konnte nachweisen, dass die klinische Untersuchung eine Sensitivität von 57,1\% und eine Spezifität von 97,1\% aufweist [Ratanachaikanont 2005]. Kösters und Gotzsche konnten keine Verringerung der Mammakarzinom-Mortalitätsrate in der Gruppe der selbstuntersuchenden bzw. klinisch untersuchten Frauen im Vergleich zu nicht untersuchten Frauen feststellen [Kösters und Gotzsche 2003]. In einer niederländischen Studie konnten nur $6 \%$ von 127 kontralateralen und $22 \%$ von 58 rezidivierenden Mammakarzinomen bei positiver Eigenanamnese palpiert werden [Lu et al. 2011]. 


\subsubsection{Stellenwert der Mammographie in der Früherkennung}

Das PGMI-System dient der Qualitätskontrolle von Mammographieaufnahmen. Die Kriterien sind standardisiert um Screeningaufnahmen, aber auch Aufnahmen im Rahmen der Befundabklärung zu beurteilen. „Perfekte“ Aufnahmen sind fehlerfrei und machten im präsentierten Kollektiv $57 \%$ aus. Die "gut" eingestellten Aufnahmen weisen geringe Handhabungs- und Entwicklungsfehler auf, zeigen geringe Asymmetrien und Hautfalten und lagen in $42 \%$ in den präsentierten Daten vor. Die "mäßigen" Mammographien konnten nur in 1\% gefunden werden. "Inadäquate“ Aufnahmen lagen im ausgewerteten Kollektiv nicht vor. Diese Ergebnisse liegen allesamt innerhalb der geforderten Grenzwerte und belegten ein hohes Niveau der Bildqualität der ausgewerteten Mammographieaufnahmen.

Die Angaben zur Rate richtig-positiver Befunde in der Mammographie reicht von 8395\%, die Rate falsch-positiver Befunde von $0,9 \%$ bis 6,5\% [Mushlin et al. 1998; Athanasiou et al. 2009]. Die Sensitivität und Spezifität werden von der Brustdichte, welche wiederum u.a. von der Hormonersatztherapie, Phase des Menstruationszyklus, Parität und Body-Mass-Index (BMI) abhängig ist, beeinflusst [Mushlin et al. 1998; Carney et al. 2003].

Die Sensitivität der Mammographie korreliert mit der Parenchymdichte, d.h. dass die Sensitivität in fettreichem Gewebe (BI-RADS-I) annähernd 100\% beträgt und bei dichtem Drüsengewebe (BI-RADS-IV) deutlich unter $90 \%$ fällt [Carney et al. 2003]. In der Pisano-Studie wurden in der Gruppe III und IV sogar Sensitivitäten von nur 42\% beschrieben [Pisano et al. 2005]. Die vorliegenden Ergebnisse zeigten, dass $80 \%$ aller invasiven Karzinome in den Dichtetypen ACR III und IV zu finden waren. In der Mammographie wurden $40 \%$ aller Entitäten in den Dichtetypen ACR III und 48\% in der Gruppe IV übersehen (MX-BI-RADS 1-3).

Die Mammographie stößt bei sehr dichtem Drüsengewebe an ihre Grenzen [Day and Warren 2000]. Vor allem bei jungen Patientinnen oder mastopathischem Gewebe ist ihre Treffsicherheit eingeschränkt. In der Studie von Pisano wurde die Häufigkeit des Dichtetypen I mit 10,5\%, des Dichtetypen II mit 42,9\%, des Dichtetypen III mit 38,7\% und die des Dichtetypen IV mit 7,5\% angegeben [Pisano et al. 2005]. In der präsentierten Studie dominierten die Dichtetypen III und IV mit jeweils $43 \%$ und $37 \%$. Anzumerken ist, dass die digitale Mammographie in der Diagnostik von prä- bzw. perimenopausalen Frauen sowie von Frauen mit heterogenem oder sehr dichtem Brustparenchym der konventionellen Mammographie überlegen war [Pisano et al. 2005].

Eine hohe Sensitivität für die Detektion von Mikrokalk im Mammogramm besteht ab einem Durchmesser von 0,1 mm [Fischer et al. 2002]. Dies ist klinisch relevant, da Mikroverkalkungen mit einem malignen Geschehen assoziiert sein können. Zirka $30 \%$ der invasiven Karzinome und 50\% der in-situ-Karzinome weisen Kalk auf [Yang und Tse 2004]. 
Nach Ikeda und Andersson zeigten allerdings nur 27\% der Mammographie-Bilder die typischen "Softmarker" eines DCIS [Ikeda und Andersson 1989], so dass nur insgesamt $60 \%$ der in-situ Karzinome durch die Mammographie allein entdeckt werden konnten [Pisano et al. 2005]. Dies deckte sich mit den Ergebnissen unserer Auswertung, in der bei BI-RADS 4 ein DCIS-Anteil von 60\% und in der BI-RADS 5Gruppe ein Anteil von 52\% ermittelt wurde. Insgesamt zeigten $68 \%$ aller duktalen Carcinoma in-situ die typischen Verkalkungen im Mammogramm auf. Andererseits waren $26 \%$ der DCIS mammographisch okkult.

In einer Untersuchung von 0`Flynn et al. wurde untersucht, ob das Ausmaß der Mikrokalzifikation und das Grading des DCIS vorhersagend für eine invasive Tumorentwicklung sein kann. Hierbei wurden über 10 Jahre lang 402 maligne Mikrokalzifikationen auf Ausdehnung und DCIS-Grading analysiert. In $71 \%$ war es histologisch ein reines DCIS, in 29\% ein DCIS mit invasiver Tumorkomponente. Das Risiko einer invasiven Karzinomentwicklung stieg mit zunehmender Cluster-Größe von 20\% (Cluster-Größe kleiner $11 \mathrm{~mm}$ ) auf 45\% (Cluster-Größe größer als $60 \mathrm{~mm}$ ). Ebenso stieg die Invasivität mit Zunahme des Gradings von 13\% für low-grade-DCIS auf $36 \%$ für high-grade-DCIS [O`Flynn et al. 2009]. Mammographisch sichtbare Verkalkungen korrelieren mit dem histologischen Subtypen des DCIS. Ausgedehnte Verkalkungen sind für gewöhnlich mit dem high-grade-DCIS korreliert. Die Kalkpartikel sind hier pleomorph, linear verästelt oder grobschollig in Verbänden, sowie duktal oder segmental angeordnet. Im Gegensatz dazu sind beim low-gradeDCIS die Verkalkungen punktförmig, identisch in der Anordnung eines Milchgangs oder Drüsenläppchens und sie haben Ähnlichkeit mit sekretorischen Verkalkungen [Barth 2006].

Durch die Mammographie allein konnten nach Kuhl et al. ein Drittel der in-situKarzinome als low-grade identifiziert werden [Kuhl et al. 2007a]. In den eigenen Auswertungen konnten in der Klassifikation der low-grade (G1)-in-situ-Karzinome zu $100 \%$ aufgrund des vorhandenen Kalks identifiziert werden, andere morphologische Kriterien waren unauffällig. Dies ist nicht überraschend, da mit höherem Grading weniger Kalzifikationen verbunden sind (siehe oben). Interessant war in diesem Kontext, dass mit beginnender Invasivität und Größenzunahme der Anteil der kalkassoziierten Prozesse weniger wurde (pT1a: 60\%; pT1b: 29\%) Dies legt den Schluss nahe, dass es im Rahmen des Tumorprogresses und der beginnenden Invasion zu einer Chemolyse mit Auflösung von Kalkpartikeln kommt.

Andere mammographische Auffälligkeiten wie Architekturstörungen, Herdbefunde oder Verdichtungen waren für die Detektion von DCIS-Tumoren von untergeordneter Bedeutung. Sie traten erwartungsgemäß zunehmend in der Gruppe der invasiven Tumoren (pT1a und pT1b) auf. Insbesondere Herde waren mit 10\% in der pT1aGruppe und mit 30\% in der pT1b-Gruppe zunehmend nachweisbar. Im Zusammenhang mit der zunehmenden Größe und der Zunahme des Tumorvolumens ist auch die ansteigende Detektionsrate der invasiven Tumoren im Ultraschall (pT1a: 29\%; pT1b: 56\%) erklärbar. 
Je schlechter der Differenzierungsgrad des DCIS (G1-G3) war, desto mehr nahm der prozentuale Anteil der falsch-negativen Befunde in der Mammographie ab (38\%; $21 \% ; 12 \%)$. Innerhalb der invasiven Tumorgruppe pT1a konnten $47 \%$ falsch-negativ zugeordnet werden. Auch in der pT1b-Gruppe überwog der prozentuale Anteil mit $55 \%$.

Die Mammographie ist in der Detektion von Mikroverkalkungen hochsensitiv, jedoch vergleichsweise unspezifisch [Bassett 1992]. In einer Multizenterstudie von Kettritz et al. wurden stereotaktische Vakuumbiopsien an 2874 Patientinnen untersucht. In 6\% wurden Biopsien an hoch verdächtigen, $85 \%$ an mäßig bis verdächtigen und $9 \%$ an wahrscheinlich benignen Läsionen durchgeführt. 58\% der Läsionen waren kleiner 10 $\mathrm{mm}$ und zeigten in 70\% Mikrokalzifikationen. In diesem Kollektiv wurden in $73 \%$ benigne Befunde erhoben [Kettritz et al. 2004]. Von 109 im diagnostischen Brustzentrum durchgeführten Biopsien entfielen 25\% auf die mammographisch gesteuerte Gewebeentnahme. Die Quote gutartiger Veränderungen, die nicht Gegenstand dieser Studie war, lag hier in einer vergleichbaren Größenordnung von $66 \%$. Der überwiegende Anteil der Biopsien mit malignem Befund (96\%) entsprach der nicht-invasiven B5a-Kategorie. Nur ein geringer Anteil von 4\% war mit einem invasiven Karzinom assoziiert. Grund hierfür ist die Tatsache, dass im Prinzip ausschließlich unklare Mikroverkalkungen stereotaktisch abgeklärt wurden.

Intervallkarzinome sind definiert als Karzinome, die im Intervall zwischen zwei Screeningrunden klinisch oder bildgebend auffallen. Unterteilt werden sie in solche Tumoren, die zum Zeitpunkt des letzten Screenings nicht zu sehen waren (",no sign“), retrospektiv doch zu erkennen sind („minimal sign") oder schlichtweg übersehen wurden („missed carcinoma“) [www.tumorzentrum-muenchen.de]. Der Anteil der jeweiligen Gruppen ist etwa gleich hoch, also zwischen 30-35\%. Es kann somit davon ausgegangen werden, dass mindestens 2 Drittel der Intervallkarzinome zum Zeitpunkt der letzten Screening-Untersuchung existent war, anhand der Mammographie jedoch nicht erkannt wurde. Dies deckte sich mit den Ergebnissen der vorliegenden Studie, in der für kleine invasive Tumorformen $24 \%$ mammographisch okkult waren.

\subsubsection{Stellenwert der Mammasonographie in der Früherkennung}

Die Mammasonographie hat ihren Stellenwert in der Früherkennung des Mammakarzinoms insbesondere bei jüngeren Frauen mit dichtem, mammographisch nur schwer beurteilbarem Parenchym. Für Frauen mit mammographischem Dichtetyp ACR III und IV erweist sich die Sonographie hinsichtlich der Sensitivität der Röntgenmammographie sogar überlegen [Kuhl et al. 2010]. Dies ist auch vor dem Hintergrund wichtig, dass z.B. dichtes Drüsengewebe bei postmenopausalen Frauen ein Marker für ein erhöhtes Mammakarzinom-Risiko und mit dem Auftreten von Intervallkarzinomen assoziiert ist [Nothacker et al. 2009]. 
In einer 2007 durchgeführten Studie von Madjar et al. wurden 24\% aller im Brustzentrum operierten Mammakarzinome im Rahmen der Früherkennung bei asymptomatischen Frauen ausschließlich sonographisch entdeckt. Diese Tumoren waren klinisch und radiologisch unauffällig [Madjar et al. 2010].

Als Nachteil der Mammasonographie werden die limitierte Sensitivität, die Abhängigkeit der Aussagekraft von der Erfahrung des Untersuchers und die Dauer der Untersuchung angesehen [Berg und Gilbreath 2000]. Zudem werden Präkanzerosen, intraduktale Tumoren und kleine invasiv wachsende Mammakarcinome (<5 mm Größe) nicht zuverlässig erfasst [Berg und Gilbreath 2000; Delorme und Debus 1998; Hackelöer et al. 1986]. Dies deckte sich mit den eigenen Ergebnissen der präsentierten Studie, in der 61\% sonographisch okkult waren. Für Tumoren bis $5 \mathrm{~mm}$ (pT1a) betrug die Sensitivität $29 \%$. Sie stieg für größere Karzinome (5-10 mm; pT1b) auf 56\%.

Hinsichtlich des Gewebetyps fanden sich im eigenen Kollektiv in $46 \%$ homogen parenchymales und in $44 \%$ inhomogenes Brustdrüsenparenchym. In diesen speziellen Gewebetypen wurde, bei vorhandenem präinvasiven oder invasiven Tumorgeschehen, mit dem Ultraschall in 50\% BI-RADS 1-Befunde und in 10\% BIRADS 2-Befunde eruiert, was nach der Klassifikation des American College of Radiology einem Normalbefund entspricht. Über $60 \%$ der sonographisch ermittelten Befunde wurden als vermeintlich gutartig gesehen. Lediglich in $17 \%$ konnte eine $\mathrm{BI}$ RADS-Kategorie 4 ermittelt werden, die mit einer Tumorwahrscheinlichkeit von 95\% einhergeht und histologisch abgeklärt werden sollte. Wiratkapun et al. untersuchten die Biopsierate und den positiven prädiktiven Wert in der BI-RADS-Kategorisierung 4, hierbei war die Biopsierate $75 \%$, davon waren $21 \%$ des Gewebes maligne. In $67 \%$ konnte ein DCIS diagnostiziert werden [Wiratkapun et al. 2010]. Nach Park et al. konnten high-grade-DCIS-assoziierte Mikrokalzifiaktionen sonographisch in nur $35,5 \%$ erkannt werden. Non-high-grade-DCIS konnte in 50\% nur aufgrund seiner irregulären hypoechogenen Tumormasse mit unscharfem mikrolobulierten Randsaum identifiziert werden [Park et al. 2010]. In der eigenen Untersuchung der zeigte sich, dass letztendlich auch nur $27 \%$ der DCIS im Ultraschall diagnostiziert wurden. Diese Quote stieg auf 29\% für Befunde im Stadium pT1a und $56 \%$ für das Stadium $\mathrm{pT} 1 \mathrm{~b}$.

Betrachtet man die Anteile der B-Kategorisierung nach der ultraschallgesteuerten Stanze, ergab dies in der vorgestellten Studie in $70 \%$ ein invasives Tumorgeschehens (B5b). 


\subsubsection{Stellenwert der Mamma-MRT in der Früherkennung}

Der Einsatz der Mamma-MRT wird immer noch kontrovers diskutiert. In Abhängigkeit vom Versicherungsstatus, der jeweils empfehlenden Fachgesellschaft sowie der Studiendaten reicht das Spektrum der anerkannten Indikationen von der alleinigen Anwendung nach brusterhaltender Therapie des Mammakarzinoms und CUPSyndrom (GKV-Indikationen) über das lokale Staging bei Patientinnen mit invasiv lobulärem Mammakarzinom sowie der Früherkennung bei Hochrisikofrauen [S3Leitlinie... Mammakarzinom 2008] bis hin zum generellen Einsatz bei sehr hoher Gewebedichte im Mammogramm [Fischer 2010].

Einerseits wird formuliert, dass die MRT eine Kombination aus Mammographie und Mammasonographie nicht ersetzen soll [Orel 2008], andererseits werden bei unauffälligem MRT-Befund bei hochwertiger Technik und Methodik eine ergänzende Mammographie und Sonographie für entbehrlich erachtet [Kuhl et al. 2010]. Der sinnvolle Einsatz der MRT im Rahmen der Diagnostik bei Frauen mit Prothesen ist unstrittig.

In der vorliegenden Arbeit wurden die MRM-Artefaktstufen I-IV berücksichtigt. Der größte Teil (93\%) der MR-Befunde ließ sich der Artefaktstufe I mit nicht vorhandenen Bewegungs-und Subtraktionsartefakten zuordnen. Bewegungsartefakte der Stufen III und IV traten in nur 1\% der Fälle auf. Die Dichte in Abhängigkeit vom Enhancement wurde ebenfalls evaluiert. Die Dichtetypen ACR I und II, die eine sehr hohe bzw. hohe Transparenz der parenchymalen Strukturen implizieren und mit einer sehr guten Beurteilbarkeit der MRT assoziiert sind, dominierten mit 89\%. Der Dichtetyp ACR II zeigte sich in $24 \%$ der Fälle und die in ihrer Aussagekraft limitierten Aufnahmen der Dichtetypen III und IV lagen in nur $11 \%$ der Fälle vor.

Mit der Mamma-MRT steht eine sensitive Untersuchungsmöglichkeit für die weibliche Brust zur Verfügung [Heinisch et al. 2003]. In der Literatur werden Sensitivitäten von bis zu 97,4\% beschrieben [Winnekendonk et al. 2004; Wurdinger et al. 2001; Kuhl et al. 2010]. Allerdings muss kritisch angemerkt werden, dass es sich bei den jüngeren Mitteilungen und den exzellenten Ergebnissen der Mamma-MRT ausschließlich um Studien mit qualitativ hochwertigen, nicht bewegungsartefaktbehafteten MRAufnahmen mit räumlich sehr hoher Auflösung (Matrix 512x512) handelt. Hauptgrund für die sehr guten Ergebnisse der MRT ist die oben aufgeführte hohe Quote an geringen Dichtetypen ACR I und ACR II, die im Gegensatz zur Mammographie unabhängig von der parenchymalen Brustdichte ist. Die frühzeitige Anreicherung von Kontrastmittel in invasiven Karzinomen und das erst später stattfindende Enhancement im umgebenden gesunden Parenchym ist der Hauptgrund für diese hohe Sensitivität. Die fehlende Kontrastmittelaufnahme in MR-mammographisch und/oder klinisch auffälligen Brustarealen hat dadurch einen hohen negativen Vorhersagewert (94\%) für invasive Mammakarzinome [Schnall et al. 2006]. Dies gilt vor allem im Zusammenhang mit postoperativen Veränderungen wie z.B. Narben [Heywang et al 1990]. 
In der präsentierten Studie konnte der größte Teil der Karzinome als MR-BIRADS 4 $(56 \%)$ und $5(37 \%)$ kategorisiert werden. Insgesamt wurden alle nachgewiesenen invasiven Mammakarzinome in der Mamma-MRT detektiert. Von den DCIS-Tumoren wurden $84 \%$ magnetresonanztomographisch richtig erkannt.

Herde dominierten in der pT1a-Gruppe mit $44 \%$ und in der pT1b-Gruppe mit $79 \%$ in der Befundung, während sie in der Evaluation der DCIS-Tumoren nur in 11 bis $24 \%$ vorkamen. Im Gegensatz hierzu bestimmten Non-mass-like-lesions in etwa $79 \%$ der Fälle mit DCIS das MR-Kriterium, während diese nicht-raumfordernden Anreicherungen mit zunehmender Invasivität und Größe mit 50\% bzw. 16\% zunehmend in den Hintergrund traten.

Foci waren in der Diagnostik der DCIS-Gruppe und invasiven Gruppe prozentual kaum präsent. Dies deckt sich mit den Aussagen von Kuhl et al., die darauf hinweisen, dass die typischen MR-Mammographie-Diagnosekriterien für invasive Karzinome von den Diagnosekriterien für präinvasive Vorstufen differieren [Kuhl et al. 2007a].

DCIS wurde in dieser Studie in $84 \%$ der Fälle als Befund der MR-BIRADS Kategorie 4 oder 5 korrekt erkannt. In der Literatur werden diesbezüglich Sensitivitäten von 58\%-93\% beschrieben [Fischer et al. 1996; Kuhl et al. 2010]. Kuhl et al. konnten widerlegen, dass die Mammographie eine höhere Sensitivität hinsichtlich des Nachweises von Mikrokalzifikation beim DCIS hat als die MR-Mammographie. Sie ermittelten weit höhere Sensitivitäten für die MRT (92\%) und hier insbesondere für high-grade-Läsionen (Sensitivität 60\%) [Kuhl et al. 2007a]. In diesem Zusammenhang wird proklamiert, dass die Hälfte aller high-grade-DCIS-Läsionen ohne Nekrosen mammographisch okkult ist [Boetes und Mann 2007]. Dies wird durch die Tatsache erklärt, dass aufgrund der Tumor-Angiogenese die Tumorgefäße bei high-grade- DCIS "reifer" sind und dadurch das typische „wash-out"-Phänomen missen. Stattdessen erscheinen asymmetrisch angereicherte Areale, die Tumorgefäßdichte ist höher und folglich die Anreicherung intensiver, was die bessere Darstellbarkeit im Vergleich zum low-grade-DCIS erklärt [Cao et al. 2004]. In den vorliegenden Auswertungen zeigte sich, dass die Rate in der MR-Mammographie okkulter DCIS mit zunehmendem Grading keine eindeutige Tendenz aufwies. Sie betrug für die nicht entdeckten DCIS-G1-Läsionen 31\%, für die G2-Läsionen 7\% und für die G3-Läsionen 13\%.

Einige Arbeitsgruppen kritisieren die geringe Spezifität der Mamma-MRT mit der Konsequenz unnötiger kurzfristiger Kontroll- und Zusatzuntersuchungen [Kriege et al. 2004]. Zur Spezifität der Mamma-MRT sei jedoch angemerkt, dass sie in ähnlicher Größenordnung liegt wie die Mammographie betreffs der Charakterisierung von Mikroverkalkungen. Fischer et al. zeigten z.B. an einem Patientengut, dass bei $27,8 \%$ der MR-gesteuerten Vakuumbiopsie ein bösartiger und bei weiteren 12,8\% ein Borderline-Befund im Sinne einer BI-RADS 3-Läsion vorlag. 59,4\% der Biopsate waren benigne [Fischer et al. 2009]. Diese Daten stimmen mit den Ergebnissen der 
bereits beschriebenen Multicenterstudie von Kettritz et al. annähernd exakt überein [Kettritz et al. 2004].

Die Überlegenheit der MRT im Nachweis kleiner Karzinome zeigt sich u.a. bei der Entdeckung zusätzlicher Malignome durch die Mamma-MRT im Rahmen des prätherapeutischen Stagings. In einer Studie von Fischer et al. resultierte hieraus eine Änderung der operativen Strategie (i.S. einer brusterhaltenden Therapie oder Mastektomie) bei $14,3 \%$ der Patientinnen [Fischer et al. 1999]. In einer Studie von Lehman et al. lag die Sensitivität der MR-Mammographie in der Detektion von kontralateralen Tumoren bei $91 \%$, die Spezifität bei $88 \%$ [Lehman et al. 2007]. Die entdeckten Karzinome waren in etwa 50\% ein duktales Carcinoma in situ [Lehman et al. 2007; Kuhl et al. 2007b], was die höhere Sensitivität der MRT im Vergleich zur Mammographie für das DCIS erneut belegt [Berg et al. 2004].

\subsubsection{Stellenwert von Mammographie und Sonographie in der Früherkennung}

Durch die Kombination der Sonographie mit der Mammographie sind bessere diagnostische Ergebnisse zu erwarten, als mit der Mammographie alleine [Zonderland et al. 1999]. Bei einer symptomatischen Frau über 35 Jahren wird zumeist als erster Schritt die Mammographie empfohlen [Heywang-Köbrunner und Schreer 2003]. Andere Empfehlungen gehen hier vom 40. Lebensjahr aus, sofern nicht ein definiertes Hochrisiko vorliegt [S3-Leitlinien... Mammakarzinom 2008]. Der Ultraschall wird anschließend gezielt zur weiteren Abklärung eines tastbaren und/oder mammographisch detektierten Tumors eingesetzt. Durch den kombinierten Einsatz von Ultraschall und Mammographie erhöht sich die Tumordetektionsrate bis zu 98\% aller mit diesen beiden Verfahren gefundenen Tumoren [Zonderland et al. 1999; Yang und Tse 2004].

Bei allen Mammakarzinomen hat die Sonographie eine Detektionsrate von unter ca. $20 \%$ [Bassett et al. 1987]. Die falsch-negative Rate wird insgesamt mit $20 \%$ angegeben [Dempsey 1988]. Die falsch-positive Rate des Ultraschalls ist nicht zu vernachlässigen. In einer Arbeit von Sickles et al. wurden bei 587 asymptomatischen Frauen mit unauffälligem Mammogramm in 13,6\% sonographisch nachgewiesene Herdbefunde beschrieben, die letztendlich nicht maligne waren [Sickles et al. 1984].

An ihre Grenzen stößt die Sonographie bei verkalkenden Malignomen und ihren Vorstufen. Mikrokalk ist nicht sichtbar, weshalb ein ausschließliches sonographisches Screening nicht möglich ist. Zeigen sich im Bereich bekannter mammographisch entdeckter Mikroverkalkungen sonographisch echoarme inhomogene Strukturen, so liegt meist bereits ein invasives Tumorstadium vor [Nishimura et al. 2004]. Bei dem die Mammographie ergänzenden Brustultraschall zeigt sich in der Gruppe von Frauen mit dichtem Brustdrüsengewebe (ACR III und IV), dass kleine Mammakarzinome gelegentlich sensitiver detektiert werden können [Nothacker et al. 2009]. 
In einer italienischen Studie stieg die Sensitivität des Ultraschalls in der Entdeckung einer Läsion von $35 \%$ bei alleiniger Untersuchung auf $83 \%$ bei Kenntnis eines mammographischen Herdbefundes [Cilotti et al. 1997]. In der EVA-Studie erreichte die alleinige Ultraschalluntersuchung eine Sensititvität von $37 \%$, die alleinige Mammographie nur 33\%. In der Kombination erreichten beide Verfahren eine Sensititvität von $48 \%$. Der positiv-prädiktive Wert lag bei beiden Verfahren annähernd gleich hoch (Mammographie: 39,1\%, Ultraschall: 35,7\%) [Kuhl et al. 2010]. In den präsentierten Daten erreichte die Mammographie in der Detektion von DCIS-Läsionen eine Sensitivität von 74\%, der Ultraschall nur 27\%. In den Gruppen der invasiven Tumoren erreichte die Mammographie bzw. der Ultraschall in der pT1a-Gruppe eine Sensitivität von 53\% bzw. 29\% und in der pT1b-Gruppe eine Sensitivität von $45 \%$ bzw. 56\%. In der Gesamtdetektion aller frühen Tumore erreichte die Mammographie eine Sensititvität von 60\%, der Ultraschall nur $39 \%$.

\subsubsection{Stellenwert von Mamma-MRT und Sonographie in der Früherkennung}

Aus den meisten Veröffentlichungen geht hervor, dass ein Teil der in der MRT detektierten Mammaläsionen in Kenntnis des Befundes bei gezielter Ultraschalluntersuchung im Sinne eines "Second-Looks" nachvollziehbar war und über die sonographisch gesteuerte perkutane Biopsie abgeklärt werden konnte [Teifke et al. 2003]. Teifke et al. und La Trenta et al. berichteten, dass vor allem maligne Herde in der retrospektiven Sonographie erkennbar waren [Teifke et al. 2003; La Trenta et al. 2003].

Das Ziel der Sekundärprävention von Brustkrebs in Hochrisikokollektiven ist die Früherkennung. Trecate et al. bestätigen die hohe Sensitivität der MRMammographie, bemängeln allerdings die niedrige Spezifität. Um unnötige Biopsien zu veranlassen, sollte die Kombination mit anderen konventionellen Verfahren angestrebt werden, insbesondere mit dem Second-look-Ultraschall, der sich als nützlich bei der Aufklärung von falsch-positiven MR-Mammographie-Befunden erwiesen hat [Trecate et al. 2006].

Die Mamma-MRT ist sensitiver in der Detektion von in-situ-Karzinomen als die Sonographie. In den präsentierten Auswertungen wurden sonographisch nur 27\% aller DCIS entdeckt. Die sonographische Zusatzuntersuchung nach einer MRMammographie bringt nach Berg et al. keinen therapeutischen Benefit [Berg et al. 2004]. Auch nach den Daten aus der EVA-Studie kann die hohe Sensitivität der MRMammographie $(93 \%)$ durch den Zusatz der Sonographie nicht gesteigert werden [Kuhl et al. 2010]. Nach unseren Daten konnte die MR-Mammographie in 84\% DCISLäsionen erkennen, der Ultraschall nur in $27 \%$ der Fälle. In den invasiven Gruppen betrug die Sensitivität jeweils $100 \%$, so dass eine zusätzliche Sonographie ohnehin überflüssig gewesen wäre. 


\subsubsection{Stellenwert von Mammographie und Mamma-MRT in der Früherkennung}

Bei invasiven lobulären und muzinösen Karzinomen und Karzinomen mit einer ausgeprägten intraduktalen Komponente ist die Sensitivität der MR-Mammographie höher als die der Mammographie [Weinstein et al. 2001]. Die Kombination von MRMammographie und Mammographie ist jedoch sensitiver als jede andere Untersuchungskombination [Berg et al. 2004].

In der EVA-Studie erreichte die Kombination von Mammographie und MRMammographie eine Sensitivität von $100 \%$ und eine Spezifität von $97,6 \%$. Der positiv-prädiktive Wert lag insgesamt bei 40,2\% [Kuhl et al. 2010]. Auch in den vorliegenden Daten zeigte sich die Überlegenheit der MR-Mammographie mit 100\% in der Detektion aller invasiven Tumoren (pT1a und pT1b). Hier wäre eine zusätzlich durchgeführte Mammographie überflüssig geworden.

Die Mamma-MRT ist sensitiver in der Entdeckung von multifokalen Tumoren als die Mammographie, insbesondere bei dichtem Brustparenchym. Allerdings gilt für beide Verfahren ein niedriger positiv-prädiktiver Wert (Mammographie 76\%, MRMammographie 68\%) [Sardanelli et al. 2004]. Auch bei Hochrisikokollektiven (familiäre oder genetische Disposition) zeigte sich die MR-Mammographie sensitiver als die Mammographie (MR-Mammographie 71,1\%, Mammographie 40\%). Die Spezifität liegt ebenfalls höher (MR-Mammographie 95\%, Mammographie 89,8\%) [Kriege et al. 2004].

In den Auswertungen zeigte sich, dass im Vergleich von Mammographie und MRMammographie $47 \%$ der falsch-negativen Befunde innerhalb der invasiven Tumorgruppe pT1a bzw. 55\% der invasiven Tumorgruppe pT1b in der Mammographie auftraten. Die MR-Mammographie konnte mit jeweils $100 \%$ sicher die invasiven Tumorgruppen eruieren. In der Differenzierung von DCIS G1-G3 zeigten sich ebenfalls Unterschiede. So konnten in der DCIS G1-Gruppe prozentual eine ähnliche Verteilung von 62\% (Mammographie) und 69\% (MR-Mammographie) gefunden werden. Mit zunehmendem Differenzierungsgrad stieg die Detektionssicherheit der MR-Mammographie zunächst auf 93\% (DCIS G2), fiel dann aber auf $87 \%$ (DCIS G3) ab. Im Vergleich dazu, hielt die Mammographie ein niedrigeres Niveau von 79\% (DCIS G2) bzw. 78\% (DCIS G3). In der Gruppe der Gesamt-DCIS zeigte sich bei der Mammographie eine Sensitivität von $74 \%$, in der MR-Mammographie eine Sensitivität von $84 \%$. 


\subsubsection{Stellenwert von Mammographie, Sonographie und Mamma-MRT in der Früherkennung}

In einer Multizenterstudie zum Screening von Hochrisikopatientinnen mittels Mammographie, Sonographie und MR-Mammographie wurden bei 2,7\% der Patienten nur MR-sichtbare suspekte Herde gefunden, die in 31,6\% maligne waren [Kuhl et al. 2005].

Die Mamma-MRT ist dem Ultraschall und der Mammographie in der Entdeckung von multizentrischen Tumoren überlegen [Sardanelli et al. 2004]. Die MR-Mammographie kann Sensitivitäten von bis zu 100\% aufweisen, wenn man eine falsch-positive Rate von $23 \%$ in Kauf nimmt [van Goethem et al. 2004]. In einer großen Studie von Fischer et al. wurden die Sensitivität, Spezifität und Trefferquote der MRT mit der klinischen Untersuchung und den bildgebenden Verfahren bei multifokal bzw. multizentrisch wachsenden Tumoren verglichen. Die Sensitivität, Spezifität und Trefferquote betrugen bei der klinischen Untersuchung $58 \%, 76 \%$, und $62 \%$ und die der Mammographie $86 \%, 32 \%$ und $72 \%$. Für die Sonographie betrug die Sensitivität $75 \%$, die Spezifität $80 \%$ und die Trefferquote $76 \%$. Trotz einer Spezifität von $65 \%$ konnte die Mamma-MRT sowohl mit $93 \%$ die höchste Sensitivität als auch mit $85 \%$ die höchste Trefferquote vorweisen [Fischer et al. 1999]. Kritisch anzumerken ist, dass dies eine sehr alte Studie und mit dem heutigen technischen Standard nicht mehr vergleichbar ist.

Sardanelli et al. untersuchten in einer großen multizentrischen Studie die Wirksamkeit von klinischer Untersuchung, MR-Mammographie, Mammographie und Sonographie in Hochrisikokollektiven ( $n=501$, Alter gleich oder höher 25 Jahre, familiärer positive Anamnese und Eigenanamnese). Die MR-Mammographie war insgesamt sensitiver $(91 \%)$ als die klinische Untersuchung (18\%), Mammographie (50\%), Ultraschall (52\%) und Ultraschall plus Mammographie (63\%). Die Spezifität reichte von $96 \%$ bis $99 \%$, der positiv-prädiktive Wert von $53 \%$ bis $71 \%$. Die MRMammographie zeigte signifikant bessere negativ-prädiktive Werte $(99,6 \%)$ als die vergleichenden Verfahren. Von 52 diagnostizierten Karzinomen wurden 31\% allein durch die MR-Mammographie erkannt, davon $26 \%$ bei Frauen unter 50 Jahren und 26\% bei Frauen mit oder über 50 Jahren [Sardanelli et al. 2011].

Eine Arbeitsgruppe von Trop et al. kam zu ähnlichen Ergebnissen, in einer prospektiven Studie verglichen sie ebenfalls bildgebenden Verfahren bei BRCA1/2Trägerinnen. Hierbei wurde eine jährliche MR-Mammographie und Mammographie, sowie ein zweimal pro Jahr durchgeführter Ultraschall gepaart mit einer klinischen Untersuchung miteinander verglichen. Die MR-Mammographie-Befunde wurden von drei unabhängigen Radiologen ausgewertet. Von insgesamt 184 Frauen wurden 12 Tumore entdeckt, davon 9 invasive und 3 in-situ-Karzinome. Die MR-Mammographie detektierte 10, die Mammographie 7, der Ultraschall entdeckte keine zusätzlichen Tumoren. Die Wiedervorstellungsrate war 21,8\% bei der MR-Mammographie, 11,4\% beim Ultraschall und $16,1 \%$ bei der Mammographie. 
Insgesamt wurden 45 Biopsien durchgeführt, 21 aufgrund von Ultraschallabnormalitäten, 17 bei MR-Mammographie und 7 bei mammographischen Auffälligkeiten. Die MR-Mammographie war hinsichtlich der Sensitivität den anderen Verfahren überlegen. Die Kombination von jährlich durchgeführter MRMammographie und Mammographie erreichte einen negativen Voraussagewert von $100 \%$ [Trop et al. 2010].

In der EVA-Studie zeigte sich, wie wichtig die MR-Mammographie in der Früherkennung geworden ist. Die Entdeckungsrate von Tumoren wurde durch die alleinige Durchführung der MR-Mammographie erhöht und war nicht signifikant höher, als es in der Kombination mit der Mammographie, dem Ultraschall oder beiden Verfahren gewesen wäre. Die Sensitivität der MR-Mammographie lag hier bei 92,6\%, die der Mammographie bei 33,3\% und die des Ultraschalls bei $37 \%$. In der Kombination von Mammographie und Ultraschall konnte eine Sensitivität von 48,1\%, bei der Kombination von MR-Mammographie und Ultraschall eine Sensitivität von 92,6\%, in der Kombination von MR-Mammographie und Mammographie eine Sensitivität von $100 \%$ und in der Kombination von allen Verfahren ebenfalls eine Sensitivität von 100\% erreicht werden [Kuhl et al. 2010].

$\mathrm{Da}$ es bisher keine Arbeiten zum Stellenwert der Methoden bei kleinen Tumoren (DCIS, pT1a und pT1b) gibt, werden in der vorliegenden Untersuchung Mammographie, Ultraschall und MR-Mammographie erstmals in der Entdeckung der präinvasiven und kleinen invasiven Tumoren bis $10 \mathrm{~mm}$ miteinander verglichen.

Mammographisch wurden insgesamt 65 Tumore (60\%) von 109 Läsionen sicher erkannt. 44 Tumoren (40\%) wurden übersehen, wovon $31 \%$ den DCIS G1, $21 \%$ den DCIS G2 und 17\% den DCIS G3-Tumoren entsprachen. In der Gruppe der invasiven Tumore wurden $47 \%$ der pT1a-Gruppe und 55\% der pT1b-Gruppe falsch-negativ befundet.

Sonographisch wurden insgesamt 40 (39\%) von 103 Läsionen sicher erkannt. 63 $(61 \%)$ wurden übersehen, wovon $73 \%$ der DCIS-Gruppe 1-3 entsprach. In der Gruppe der invasiven Tumore wurden 71\% der pT1a-Gruppe und 44\% der pT1bGruppe falsch-negativ befundet.

In der Mamma-MRT wurden 101 (93\%) von 109 Läsionen sicher erkannt. 8 Tumoren (7\%) wurden übersehen, wovon $31 \%$ den DCIS G1, 7\% den DCIS G2 und $13 \%$ den DCIS G3-Tumoren entsprachen. In der Gruppe der invasiven Tumore wurden 0\% der pT1a-Gruppe und 0\% der pT1b-Gruppe falsch-negativ befundet.

Im Vergleich der drei Verfahren zeigte sich, dass die Mamma-MRT insbesondere für invasive Karzinome, letztendlich aber auch für alle intraduktalen Tumoren sensitiver ist, als dies mit dem Ultraschall oder der Mammographie möglich ist. $93 \%$ der Befunde wurden damit als abklärungsbedürftig oder tumorverdächtig eingestuft. Im Vergleich hierzu wurden nur 60\% der Tumore durch die Mammographie und nur 39\% durch den Ultraschall als richtig-positiv eingeschätzt. 


\subsubsection{Perspektiven}

Die Mammographie galt bislang als das wichtigste Verfahren in der Mammadiagnostik, sowohl für die Tumorfrüherkennung als auch für die Tumornachsorge. Die generellen Vorteile in der Früherkennung sind der geringe zeitliche Aufwand sowie die Reproduzierbarkeit und Dokumentierbarkeit dieser Methode. Abgesehen von den Anschaffungskosten ist sie im Vergleich zu schnittbildgebenden Untersuchungsverfahren wie CT und MRT ein kostengünstigeres Verfahren.

Die Mammographie ist aufgrund des zuverlässigen Nachweises von Mikroverkalkungen sensitiv für die Detektion von in-situ Karzinomen [Kuhl et al. 2007a].

In der präsentierten Studie konnten DCIS-Tumore anhand des Kriteriums der Verkalkungen mit 100\% in der DCIS G1-Gruppe, mit 82\% in der DCIS G2-Gruppe und mit 95\% in der DCIS G3-Gruppe entdeckt werden. Im Vergleich zur MammaMRT war sie allerdings in der Entdeckung sämtlicher Formen der in-situ Läsionen unterlegen. So detektierte sie 74\% aller DCIS und die MRT 84\% aller präinvasiven Vorstufen. Unberücksichtigt bleibt in dieser Diskussion, inwieweit das Auffinden von DCIS vom low-grade-Typ eine klinische Relevanz besitzt bzw. das Langzeitüberleben der entsprechenden Patientin beeinflusst, da immerhin etwa $70 \%$ dieser Tumoren zeitlebens nicht invasiv werden und keine Bedrohung darstellen.

In der Zusammenschau der präsentierten Daten ist daher die Frage naheliegend, ob das Konzept einer alleinigen Mamma-MRT zur frühen Erkennung von Brustkrebs ausreichend ist oder ob eine ergänzende Mammographie bzw. Sonographie zu einer relevanten Verbesserung der Ergebnisse führen würde. Von Bedeutung erscheint in diesem Zusammenhang, dass die zuverlässige Diagnose invasiver Tumoren in einem frühen Stadium unbestritten von hoher Bedeutung ist, während das Erkennen von intraduktalen Tumorstadien hinsichtlich des Langzeitüberlebens durchaus kontrovers diskutiert wird. Es stellt sich hier zudem die Frage, ob eine regelmäßige bildgebende Diagnostik in adäquaten Intervallen von 1-2 Jahren eventuell ausreichend ist, um den potentiellen Übergang präinvasiver in invasive Tumorstadien zuverlässig zu erfassen. Hierzu liegen bisher keine Studiendaten vor.

Die bisherigen Daten, die sich mit den Mitteilungen anderer Autoren decken, legen den Schluss nahe, die alleinige Mamma-MRT als Screening-Verfahren einzusetzen. Hierfür sprechen die exzellente Sensitivität, die mit anderen Verfahren vergleichbare Spezifität und die fehlende Strahlenexposition. Allerdings gibt es zahlreiche Gründe, die einen populationsbezogenen Einsatz der MRT zum gegenwärtigen Zeitpunkt verbieten. Hierzu gehören die sehr hohen Kosten, die fehlende flächendeckende Verfügbarkeit des Verfahrens, die fehlende Qualitätssicherung und die nicht ausreichende Anzahl an Untersuchern mit entsprechender Expertise. Diesbezüglich gibt es gegenwärtig lediglich formulierte Ansätze zur Optimierung der Qualität und zur Kostenreduktion des Verfahrens [Fischer et al. 2012]. 


\section{Zusammenfassung}

Die frühe Diagnose des Mammakarzinoms im Stadium pTis, pT1a und pT1b erlaubt eine exzellente Prognose für das Langzeitüberleben. Zur Früherkennung stehen Mammographie (MX), Ultraschall (US) und Mamma-MRT (MR) als effektive Untersuchungsverfahren zur Verfügung.

In einer retrospektiven Auswertung an einem Kollektiv von 109 (Ultraschall: 103) frühen bzw. kleinen Karzinomen aus dem Zeitraum von 2003 bis 2011 wurden die Sensitivitäten von Mammographie, Ultraschall und Mamma-MRT ausgewertet. Das Kollektiv bestand aus 50 DCIS (45), 17 (17) Karzinomen im Stadium pT1a und 42 (41) Tumoren im Stadium pT1b. Die Untergliederung der 50 DCIS-Läsionen zeigte Befunde mit Grading 1 in 13 Fällen, Grading 2 in 14 Fällen und Grading 3 in 23 Fällen.

Die Qualität von MX und MR war exzellent. So fanden sich MX mit der Bewertung „perfekt" und "gut" in 99\% der Fälle. Bewegungsartefaktstufe I und II lagen in der MR ebenfalls in $99 \%$ vor. Die Rate qualitativ limitierter Aufnahmen betrug für beide Verfahren je $1 \%$.

Im gesamten Kollektiv der Früherkennung zeigte MX 65/109 (60\%), US 40/103 (39\%) und MRT 101/109 (93\%) der Tumoren. DCIS wurde mit MX in 37/50 (74\%), mit US in 12/45 (27\%) und in der MR in 42/50 (84\%) gefunden. Führendes Kriterium des DCIS im MX waren Mikroverkalkungen, für die MR waren es die sogenannten Non-mass-like lesions. Invasive Karzinome fanden sich in der MX in 28/59 (48\%), im US in 28/58 (48\%) und in der MRT in allen 59 Fällen (100\%). Mit zunehmender Tumorgröße stellte der Herdbefund das führende Kriterium in der Bildgebung dar.

Eine wesentliche Ursache für das deutlich bessere Abschneiden der MR gegenüber der MX lag im jeweiligen Dichtetyp der abgebildeten Bruststrukturen. Die limitierten Dichtetypen ACR III und IV fanden sich in der MX in $80 \%$ der Fälle, in der MR jedoch für das identische Kollektiv in nur $11 \%$ der Fälle. Für beide Verfahren konnte gezeigt werden, dass die Zuverlässigkeit des Verfahrens mit steigender Dichte abnimmt.

Für alle Untergruppen der DCIS-Tumoren (G1, G2, G3) zeigte die MR bessere Ergebnisse als MX und US. Die MX in Ergänzung zur MR hätte 4 G1-DCIS, 1 G2DCIS und 3 G3-DCIS mehr detektiert als die alleinige MR.

Alle klinisch relevanten Karzinome (pT1a, pT1b) wurden in der Mamma-MRT erkannt. Die zusätzliche Information von MX oder US hätte hier nicht zu einer Verbesserung der Ergebnisse führen können. MX und US detektierten in dieser Gruppe für sich gesehen nur etwa $50 \%$ der Karzinome.

Die Ergebnisse belegen, dass die MR allen anderen Verfahren (MX, US) für alle Tumorstadien innerhalb der Früherkennung des Mammakarzinoms hinsichtlich der Sensitivität überlegen ist. 
Mit Blick auf die zudem fehlende Strahlenexposition im Vergleich zur MX legt dies formal den Gedanken nahe, die MR-Mammographie als flächendeckendes Screeningverfahren einzusetzen. Hohe Kosten, begrenzte Verfügbarkeit, fehlende flächendeckende Qualität und die begrenzte Anzahl an Ärzten mit adäquater Erfahrung stehen diesem Gedanken jedoch zum gegenwärtigen Zeitpunkt entgegen. 


\section{Literatur}

Albert US, Altland H, Duda V, Engel J, Geraedts M, Heywang-Köbrunner S, Hölzel D, Kalbheim E, Koller M, König K, Kreienberg R, Kühn T, Lebeau A, Nass-Griegoleit I, Schlake W, Schmutzler R, Schreer I, Schulte H, Schulz-Wendtland R, Wagner U, Kopp I (2009): 2008 update of the guideline: early detection of breast cancer in Germany. J Cancer Res Clin Oncol 135: 339-354

American College of Radiology (ACR): Illustrated Breast imaging reporting and data system (BI-RADS). Fourth Edition. American College of Radiology, Reston [VA] 2003

Arbeitsgemeinschaft für gynäkologische Onkologie E.V: Aktuelle Empfehlungen zur Prävention, Diagnostik und Therapie primärer und fortgeschrittener Mammakarzinome. Zuckschwerdt Verlag, München, Bern, Wien, New York 2011

Arnerlöv C, Emdin SO, Roos G, Angström T, Bjersing L, Ängquist K-A, Jonsson H (1990): Static and flow cytometric DNA analysis compared to histologic prognostic. Eur J Surg Oncol 16: 200-208

Arriagada R, Rutqvist LE, Skoog L, Johansson H, Kramar H (1992): Prognostic factors and natural history in lymph node-negative breast cancer patients. Breast Cancer Res Treat 21: 101-109

Athanasiou A, Tardivon A, Ollivier L, Thibault F, El Khoury C, Neuenschwander S (2009): How to optimize breast ultrasound. Eur Radiol 69: 6-13

Atkinson EN, Brown BW, Montague ED (1986): Tumor volume, nodal status, and metastasis in breast cancer in women. J Natl Cancer Inst 76: 171-178

Baldwin P (2009): Digital breast tomosynthesis. Radiol Technol 81: 57-74

Bardou VJ, Arpino G, Elledge RM, Osborne CK, Clark GM (2003): Progesterone receptor status significantly improves outcome prediction over estrogen receptor status alone for adjuvant endocrine therapy in two large breast cancer databases. J Oncol 21: 1973-1979

Bartelink H, Horiot JC, Poortmans P, Struikmans H, Van den Bogaert W, Barillot I, Fourquet A, Borger J, Jager J, Hoogenraad W, Collette L, Pierart M; European Organization for Research and Treatment of Cancer Radiotherapy and Breast Cancer Groups (2001): Recurrence rates after treatment of breast cancer with standard radiotherapy with or without additional radiation. N Engl J Med $\underline{345}$ : 13781387

Barth V : Atlas der Mammadiagnostik. 2. Auflage,Thieme Verlag, Stuttgart, New York 2006

Barth V, Prechtel K: Atlas der Brustdrüse und ihre Erkrankungen. Enke Verlag, Stuttgart 1990 
Bassett LW (1992): Mammographic analysis of calcifications. Radiol Clin North Am 30: 93-105

Bassett LW, Kimme-Smith C, Sutherland LK, Gold RH, Sarti D, King W 3rd (1987): Automated and hand-held breast US: effect on patient management. Radiology $\underline{165}$ : 103-108

Bässler R: Mamma. In: Pathologie, Band 3, Springer Verlag, Berlin 1984

Bässler R, Böcker W, Hermanek P, Pickartz H, Prechtel K, Schauer A, Schnürch HG, Stegner HE (1992): Current status of grading in breast cancer. Pathologe 13: 130134

Bastert G: Malignome der Mamma. In: Schmidt-Matthiesen H (Hrsg.) Klinik für Frauenheilkunde und Geburtshilfe, Bd.12, 3.Auflage, Urban und Schwarzenberg, München, Wien, Baltimore 1990

Berg WA, Gilbreath PL (2000): Multicentric and Multifocal Cancer: Whole Breast US in Preoperative Evaluation. Radiology 214: 59-66

Berg WA, Gutierrez L, NessAiver MS, Carter WB, Bhargavan M, Lewis RS, Loffe OB (2004): Diagnostic accuracy of mammography, clinical examination, US, and MR imaging in preoperative assessment of breast cancer. Radiology 233: 830-849

Bloom HJ, Richardson WW (1957): Histological grading and prognosis in breast cancer; a study of 1409 cases of which 359 have been followed for 15 years. $\mathrm{Br} \mathrm{J}$ Cancer 11: 359-377

Boetes C, Mann RM (2007): Ductal carcinoma-in-situ and breast MRI. Lancet $\underline{370}$ : $559-460$

Boetes C, Mus RDM, Holland R, Barentsz JO, Strijk SP, Wobbes T, Hendriks J, Ruys $S$ (1995): Breast tumors. Comparative Accuracy of MR Imaging Relative to Mammography and US for Demonstrating extent. Radiol 197: 743-747

Bonadonna G, Valagussa P, Moliterni A (1995): Adjuvant cyclophosphamide, methotrexate and fluorouracil in node- positive breast cancer: the results of 20 years follow-up. N Engl J Med 332: 901-906

Bonnier P, Romain S, Charpin C, Lejeune C, Tubiana N, Martin PM, Piana L (1995): Age as a prognostic factor in breast cancer: relationship to pathologic and biologic features. Int J Cancer 62: 138-144

Borger J, Kemperman H, Hart A, Peterse H, van Dongen J, Bartelink H (1994): Risk factors in breast-conservation therapy. J Clin Oncol 12: 653-660

Botteri E, Bagnardi V, Goldhirsch A, Viale G, Rotmensz N (2010): Axillary lymph node involvement in women with breast cancer: does it depend on age? Clin Breast Cancer 10: 318-321 
Bubendorf L, Feichter GE, Obermann EC, Dalquen P: Pathologie, Begründet von W. Remmele. Zytopatholgie, Springer Verlag, 3. Auflage, Heidelberg, Berlin 2011

Cao Y, Paner GP, Kahn LB, Rajan PB (2004): Noninvasive carcinoma of the breast: angiogenesis and cell proliferation. Arch Pathol Lab Med 128: 893-896

Carney PA, Miglioretti DL, Yankaskas BC, Kerlikowske K, Rosenberg R, Rutter CM, Geller BM, Abraham LA, Taplin SH, Dignan M, Cutter G, Ballard-Barbash R (2003): Individual and combined effects of age, breast densitiy, and hormone replacement therapy use on the accuracy of screening mammography. Ann Intern Med 138: 168175

Carter CL, Allen C, Henson DE (1989): Relation of tumor size, lymph node status and survival in 24.740 breast cancer cases. Cancer $\underline{63}$ : 181-187

Chang HR (2010): Trastuzumab-based neoadjuvant therapy in patients with HER2positive breast cancer. Cancer 116: 2856-2867

Chia S, Bryce C, Gelmon K (2005): The 2000 EBCTCG overview: a widening gap. Lancet 365: 1665-1666

Cilotti A, Bagnolesi P, Moretti M, Gibilisco G, Bulleri A, Macaluso AM, Bartolozzi C (1997): Comparison of the diagnostic performance of high-frequency ultrasound as a first- or second-line diagnostic tool in non-palpable lesions of the breast. Eur Radiol 7: 1240-1244

Clark GM, Osborne CK, McGuire WL (1984): Correlations between estrogen receptor, progesterone receptor, and patient characteristics in human breast cancer. J Clin Oncol 2: 1102-1109

Clarke MJ (2008): WITHDRAWN: Multi-agent chemotherapy for early breast cancer. Cochrane Database Syst Rev 8: CD000487

Colditz GA (1993): Epidemiology of breast cancer: Findings from Nurses 'Health Study. Cancer 71: 1480-1489

Cuzick J, Sestak I, Baum M, Buzdar A, Howell A, Dowsett M, Forbes JF; ATAC/LATTE investigators (2010): Effect of anastrozole and tamoxifen as adjuvant treatment for early-stage breast cancer: 10-year analysis of the ATAC trial. Lancet Oncol 11: 1135-1141

Day N, Warren R (2000): Mammographic screening and mammographic patterns. Breast Cancer Res 2: 247-251

Delorme S, Debus J: Ultraschalldiagnostik, Hippokrates Verlag, Stuttgart 1998

Dempsey PJ (1988): The importance of resolution in the clinical application of breast sonography. Ultrasound Med Biol 1: 43-48 
Dietzel M, Baltzer PA, Vag T, Zoubi R, Gröschel T, Burmeister H, Gajda M, Runnebaum IB, Kaiser WA (2011): Potential of MR Mammography to Predict Tumor Grading of Invasive Breast Cancer. Röfo Fortschr Geb Röntgenstr Neuen Bildgeb Verf 183: 826-833

Donegan WL (1992): Prognostic factors. Stage and receptor status in breast cancer. Cancer 70: 1755-1764

Dronkers DJ, Hendriks JHCL, Holland R, Rosenbusch G: Radiologische Mammadiagnostik. Thieme Verlag, Stuttgart, New York 1999

Early Breast Cancer Trialists Collaborative Group (1996): Ovarian ablation in early breast cancer. Overview of randomized trials. Lancet $\underline{348}$ : 1189-1198

Engel J, Hölzel D, Kerr J, Schubert-Fritschle G: Epidemiologie. Manual Mammacarcinome, Tumorzentrum München. 9. Auflage. Zuckschwerdt Verlag, München 2003

Engel J, Kerr J, Hölzel D (2002): Breast Screening row overlooks basic medical facts. Breast 11: 460-461

Fisher B, Redmond C, Fisher ET, Caplan R and other Contributing National Surgical Adjuvant Breast and Bowel Project Investigators (1988): Relative worth of estrogen or progesterone receptor and pathologic characteristics of differentiation as indicators of prognosis in node negative breast cancer cancer patients:findings from National Surgical Adjuvant Breast and Bowel Project protocol B.06. J Clin Oncol ㅁ: 1076-1087

Fisher DE, Schnitt SJ, Christian R, Harris JR, Henderson (1993): Chest wall recurrence of ductal carcinoma in situ of the breast after mastectomy. Cancer $\underline{71}$ : 3025-3028

Fischer U: Lehratlas der MR-Mammographie: Hochauflösende Mamma-MRT. Thieme Verlag, Stuttgart, New York 2010

Fischer U, Baum F: Moderne Mammadiagnostik, Bayer Health Care, Leverkusen 2009

Fischer U, von Heyden D, Vosshenrich R, Vieweg I, Grabbe E (1993): Signalverhalten maligner und benigner Läsionen in der dynamischen 2D-MRT der Mamma. Röfo Fortschr Geb Röntgenstr Neuen Bildgeb Verf 158: 287-292

Fischer U, Westerhof JP, Brinck U, Korabiowska M, Schauer A, Grabbe E (1996): Das duktale In-situ-Karzinom in der dynamischen MR-Mammographiebei 1,5 T. Fortschr Roentgenstr 164: 290-294

Fischer U, Kopka L, Grabbe E (1999): Breast Carcinoma: Effect of preoperative Contrast-enhanced MR Imaging on the therapeutic Approach. Radiology 213: 881888 
Fischer U, Baum F, Obenauer S, Luftner-Nagel S, von Heyden D, Vosshenrich R, Grabbe E (2002): Comparative study in patients with microcalcifications: full-field digital mammography vs screen-film mammography. Eur Radiol 12: 2679-2683

Fischer U, Baum F, von Heyden D : Trainer Mammadiagnostik, Fallsammlung, Thieme Verlag, Stuttgart, New York 2005

Fischer U, Schwethelm L, Baum F, Luftner-Nagel S, Teubner J (2009): Aufwand, Zuverlässigkeit und histologische Ergebnisse der MR-gesteuerten Vakuumbiopsie suspekter Mammabefunde- retrospektive Auswertung von 389 Interventionen. Fortschr Röntgenstr 181: 774-781

Fischer U, Korthauer A, Baum F, Luftner-Nagel S, Heyden D, Marten-Engelke K (2012): Short first-pass MRI of the breast. Acta Radiol (e-print ahead).

Folkman J, Klagsbrun M (1987): Angiogenic factors. Science 235: 442-447

Fornage BD (2000): Recent advances in breast sonography. JBR-BTR $\underline{3}$ : 75-80

Förster A, Schreiber M, Köhler K (1998): Das bilaterale Mammacarcinom- eine retrospektive Analyse von 27 Patientinnen mit Fallbeschreibung. Röntgenpraxis $\underline{51}$ : 32-38

Frykberg ER, Bland KI (1994): Overview of the biology and management of ductal carcinoma in situ of the breast. Cancer $\underline{74}$ : 350-361

Gage I, Schnitt SJ, Nixon AJ, Silver B, Recht A, Troyen SL, Eberlein T, Love SM, Gelman R, Harris JR, Connolly JL (1996): Pathologic margin involvement and the risk of recurrence in patients treated with breast conserving therapy. Cancer $\underline{78}$ : 1921-1928

Garne JP, Aspegren K, Linell F, Rank F, Ranstam J (1994): Primary prognostic factors in invasive breast cancer with special reference to ductal carcinoma and histologic malignancy grade. Cancer $\underline{73}$ : 1438-1448

Gianni L, Eiermann W, Semiglazov V, Manikhas A, Lluch A, Tjulandin S, Zambetti M, Vazquez F, Byakhow M, Lichinitser M, Climent MA, Ciruelos E, Ojeda B, Mansutti M, Bozhok A, Baronio R, Feyereislova A, Barton C, Valagussa P, Baselga J (2010): Neoadjuvant chemotherapy with trastuzumab followed by adjuvant trastuzumab versus neoadjuvant chemotherapy alone, in patients with HER2-positive locally advanced breast cancer (the NOAH trial): a randomised controlled superiority trial with a parallel HER2-negative cohort. Lancet 375: 377-384

Goussia AC, Stefanou DG, Karaiossifidi EC, Agnantis NJ (2006): DCIS histopathology from a historical perspective. Eur J Gynaecol Oncol 27: 282-285

Hackelöer BJ, Duda V, Lauth G: Ultraschallmammographie. Springer Verlag, Heidelberg, Berlin 1986 
Heinisch M, Gallowitsch H J, Mikosch P, Kresnik E, Kumnig G, Gomez I, Lind P, Umschaden HW, Gasser J, Forsthuber EP (2003): Comparison of FDG-PET and dynamic contrast-enhanced MRI in the evaluation of suggestive breast lesions. Breast 12: 17-22

Henderson IC (2011): Can we abandon anthracyclines for early breast cancer patients? Oncology 25: 115-124, 127

Henderson IC, Berry DA, Demetri GD, Cirrincione CT, Goldstein LJ, Martino S, Ingle JN, Cooper MR, Hayes DF, Tkaczuk KH, Fleming G, Holland JF, Duggan DB, Carpenter JT, Frei E 3rd, Schilsky RL, Wood WC, Muss HB, Norton L (2001): Improved outcomes from adding sequential Paclitaxel but not from escalating Doxorubicin dose in adjuvant chemotherapy regimen for patients with node-positive primary breast cancer. J Clin Oncol 21: 976-983

Heywang SH, Hilbertz T, Beck R, Bauer WM, Eiermann W, Permanetter W. (1990): Gd-DTPA enhanced MR imaging of the breast in patients with postoperative scarring and silicon implants. J Comput Assist Tomogr 14: 348-356.

Heywang-Köbrunner SH, Schreer I: Bildgebende Mammadiagnostik. 2. Auflage, Thieme Verlag, Stuttgart, New York 2003

Holland R, Peterse JL, Millis RR, Eusebi V, Faverly D, van de Vijver MJ, Zafrani B (1994): Ductal carcinoma in situ: a proposal for a new classification. Semin Diagn Pathol 11: 167-180

Howard F, Scott-Findlay S (2006): Breast self-examination: when research contradicts accepted practice. AWHONN Lifelines 10: 66-70

Ikeda DM, Andersson I (1989): Ductal carcinoma in situ: atypical mammographic appearances. Radiology 172: 661-666

Jayasinghe UW, Taylor R, Boyages J (2005): Is age at diagnosis an independent prognostic factor for survival following breast cancer? ANZ J Surg 75: 762-762

Jörn H, Rath W: Power-Dopplersonographie (2000): eine neue vielversprechende Methode zur Beurteilung der Organdurchblutung-erste Ergebnisse in Gynäkologie und Geburtshilfe. Geburtshilfe Frauenheilkd 60: 117-124

Kato T, Kameoka S, Kimura T Nishikawa T, Kobayashi M (2003): The combination of angiogenesis and blood versal invasion as a prognostic indicator in primary cancer. Br J Cancer 88: 1900-1908

Kaufmann W, von Minckwitz G, Eiermann W, Hilfreich J, Jonat W, Kreienberg R (2004): Therapie primärer Mammakarzinome: Ergebnisse der Konferenz in St. Gallen 2003. Dtsch Arztebl 101: A-190 / B-163 / C-162

Kett K, Szilagyi K, Anga B, Kett AG, Kiralyfalvi K (2002): Axillary lymph drainage as a prognostic factor of survival in breast cancer. Lymphology 4: 161-170 
Kettritz U, Rotter K, Schreer I, Murauer M, Schulz-Wendtland R, Peter D, HeywangKöbrunner SH (2004): Stereotactic vacuum-assisted breast biopsy in 2874 patients: a multicenter study. Cancer 100: 245-251

Khan A, Newman LA (2004): Diagnosis and management of ductal carcinoma in situ. Curr Treat Options Oncol 2: 131-144

Knörr K, Knörr-Gärtner H, Beller FK, Lauritzen C: Geburtshilfe und Gynäkologie, Physiologie und Pathologie der Reproduktion. 3. Auflage, Springer Verlag, Berlin, Heidelberg 1989

Konecny G, Kahlert S, Sattler D, Kuhn W, Untch M: Neoadjuvante Chemotherapie des Mammakarzinoms. In: Untch M, Konecny G, Sittek H, Kessler M, Reiser M, Hepp $\mathrm{H}$ : Diagnose und Therapie des Mammakarzinoms- State of the Art -Zuckschwerdt Verlag GmbH, München, Bern, Wien, New York 2000, 323-335

Kopans D, Gavenonis S, Halpern E, Moore R (2011): Calcifications in the Breast and Digital Breast Tomosynthesis. Breast J 17: 638-644

Kösters JP, Gotzsche PC (2003): Regular self-examination or clinical examination for early detection of breast cancer. Cochrane Database of Systematic Reviews (2) Article ID CD003373

Kreienberg $\mathrm{R}$, Runnebaum IB, Beck $\mathrm{T}$ : Präoperative Diagnostik und operative Therapie des Mammacarcinoms. In: Kreienberg R., Möbus V., Alt D. (Hrsg.): Management des Mammmacarcinoms. Springer Verlag, Berlin, Heidelberg 1998, 133-150

Kreienberg R, Jonat W, Volm T, Möbus V, Alt D: Management des Mammakarzinoms. 3. Auflage, Springer Verlag, Berlin, Heidelberg 2006

Kriege M, Brekelmans CT, Boetes C, Besnard PE, Zonderland HM, Obdeijn IM, Manoliu RA, Kok T, Peterse H, Tilanus-Linthorst MM, Muller SH, Meijer S, Oosterwijk JC, Beex LV, Tollenaar RA, de Koning HJ, Rutgers EJ, Klijn JG; Magnetic Resonance Imaging Screening Study Group (2004): Efficacy of MRI and mammography for breast-cancer screening in women with a familial or genetic predisposition. N Engl J Med 351: 427-437

Kroman N, Jensen MB, Wohlfahrt J, Mouridsen HT, Andersen PK, Melbye M (2000): Factors influencing the effect of age on prognosis in breast cancer: population based study. BMJ $\underline{320}$ : 474-478

Kubli F, Nagel GA, Kadach U, Kaufmann $M$ : Neue Wege in der Brustkrebsbehandlung. (Aktuelle Onkologie 8). Zuckschwerdt Verlag, München, Bern, Wien 1984 
Kuhl C, Bieling HB, Gieseke J, Kreft BP, Sommer T, Lutterbey G, Schild HH (1997): Healthy premenopausal breast parenchyma in dynamic contrast-enhanced MR imaging of the breast: normal contrast medium enhancement and cyclical-phase dependency. Radiology 203: 137-144

Kuhl C, Schrading S, Weigel S, Nüssle-Kügele K, Sittek H, Arand B, Morakkabati N, Leutner C, Tombach B, Nordhoff D, Rieber A, Heindel W, Brambs HJ, Reiser M, Schild H (2005): Die „EVA“-Studie: Evaluierung der Leistungsfähigkeit diagnostischer Verfahren (Mammographie, Sonographie, MRT) zur sekundären und tertiären Prävention des familiären Mammakarzinoms - Zwischenergebnisse nach der ersten Hälfte der Förderungsperiode. Fortschr Roentgenstr 177: 818-827

Kuhl C, Schrading S, Bieling HB, Wardelmann E, Leutner CC, Koenig R, Kuhn W, Schild HH (2007a): MRI for diagnosis of pure ductal carcinoma in situ: a prospective observational study. Lancet 370: 485-492

Kuhl C, Kuhn W, Braun M Schild H (2007b): Pre-operative staging of breast cancer with breast MRI: one step forward, two steps back? Breast 16: 34-44

Kuhl C, Weigel S, Schrading S, Arand B, Bieling H, König R, Tombach B, Leutner C, Rieber-Brambs A, Nordhoff D, Heindel W, Reiser M, Schild HH (2010): Prospective Multicenter cohort Study to Refine Management Recommendations for Women at Elevated Familial Risk of Breast Cancer: The EVA Trial. J Clin Oncol 28: 1441-1445

LaTrenta LR, Menell JH, Morris EA, Abramson AF, Dershaw DD, Liberman L (2003): Breast lesions detected with MR imaging: utility and histopathologic importance of identification with US. Radiology 227: 856-861

Lebeau A (2006): Prognostic factors in ductal carcinoma in situ. Pathologe 27: 326336

Lehman CD, Gatsonis C, Kuhl CK, Hendrick RE, Pisano ED, Hanna L, Peacock S, Smazal SF, Maki DD, Julian TB, DePeri ER, Bluemke DA, Schnall MD; ACRIN Trial 6667 Investigators Group (2007): MRI evaluation of the contralateral breast in women with recently diagnosed breast cancer. N Engl J Med 356: 1295-1303

Le-Petross HT, Cristofanilli M, Carkaci S, Krishnamurthy S, Jackson EF, Harrell RK, Reed BJ, Yang WT (2011): MRI features of inflammatory breast cancer. AJR Am J Roentgenol 197: 769-776

Levine MN, Bramwell VH, Pritchard KI et al. (1998): Randomized trial of intensive cyclophosphamide, epirubicin and fluorouracil chemotherapy compared with cyclophosphamide, methotrexate and fluorouracil in premenopausal women with node-positive breast cancer. National Cancer Institute of Canada Clinical Trialists Group. J Clin Oncol 16: 2651- 2658

Liu H, Tan H, Cheng Y, Zhang X, Gu Y, Peng W (2010): Imaging findings in mucinous breast carcinoma and correlating factors. Eur J Radiol 80: 706-712 
Lorenz W, Ollenschläger G, Geraedts M, Gerlach F, Gangjour A, Helou A, Kirchner H, Koller M, Lauterbauch W, Reinauer H, Sitter H, Thomeczek C (2001): Das Leitlinien Manual: Entwicklung und Implementierung von Leitlinien in der Medizin. ZaeFQ 95: 1-84

Lu W, de Bock GH, Schaapveld M, Baas PC, Wiggers T, Jansen L (2011): The value of routine physical examination in the follow up of women with a history of early breast cancer. Eur J Cancer 47: 676-682

Madjar H, Becker S, Doubek K, Horchler T, Mendoza M, Moisidis-Tesch C, Näther B, Niebling K, Pröls U, Schardt AR, Ulrich S, Zahn U (2010): Impact of breast ultrasound screening in gynecological practice. Ultraschall Med 31: 289-95

Mammakarzinom: Manual Diagnostik und Therapie. Hrsg: A.C. Regierer, K. Possinger. Deutscher Ärzteverlag, Köln 2005

Morris EA (2002): Breast cancer imaging with MRI. Radiol Clin North Am 40: 443-466

Mouridsen H, Giobbie-Hurder A, Goldhirsch A, Thürlimann B, Paridaens R, Smith I, Mauriac L, Forbes JF, Price KN, Regan MM, Gelber RD, Coates AS. BIG 1-98 Collaborative Group (2009): Letrozole therapy alone or in sequence with tamoxifen in women with breast cancer. N Engl J Med 361: 766-776

Mushlin AL, Kouides RW, Shapiro DE (1998): Estimating the accuracy of screening mammography: a meta-analysis. Am J Prev Med 14: 143-153

Nishimura S, Takahashi K, Gomi N, Tada K, Makita M, Tada T, Iwase T, Yoshimoto M, Akiyama F, Sakamoto G, Kasumi $F$ (2004): What is the predictor for invasion in non-palpable breast cancer with microcalcifications? Breast Cancer 11: 49-54

Nothacker M, Duda V, Hahn M, Warm M, Degenhardt F, Madjar H, Weinbrenner S, Albert US (2009): Early Detection of breast cancer: benefits and risks of supplemental breast ultrasound in asymptomatic women with mammographically dense breast tissue. A systematic review. BMC Cancer $\underline{9}$ : 335

O'Flynn EA, Morel JC, Gonzalez J, Dutt N, Evans D, Wasan R, Michell MJ (2009): Prediction of the presence of invasive disease from the measurement of extent of malignant microcalcification on mammography and ductal carcinoma in situ grade at core biopsy. Clin Radiol 64: 178-183

Orel SG (2008): Who should have breast magnetic resonance imaging evaluation. J Clin Oncol 26: 703-711

Osborne CK (1990): Prognostic factors in breast cancer. Princ Pract Oncol 4: 1-11

Oven Ustaalioglu BB, Bilici A, Kefeli U, Seker M, Yildirim E, Salepci T, Oncel M, Kement M, Gumus M (2010): Does the metastatic lymph node ratio influence the disease-free survival of patients with breast cancer: single-center experiences. Oncology 79: 105-111 
Park JS, Park YM, Kim EK, Kim SJ, Han SS, Lee SJ, In HS, Ryu JH (2010): Sonographic findings of high-grade and non-high-grade ductal carcinoma in situ of the breast. J Ultrasound Med 29: 1687-1697

Pisano ED, Gatsonis C, Hendrick E, Yaffe M, Baum JK, Acharyya S, Conant EF, Fajardo LL, Bassett L, D'Orsi C, Jong R, Rebner M (2005): Diagnostic Performance of Digitial versus Film Mammography for Breast-Cancer Screening. N Engl J Med 353: 1773-1783

Pluda JM (1997): Tumor-associated angiogenesis: mechanisms, clinical implications, and therapeutic strategies. Semin Oncol 24: 203-218

Possinger K, Flath B: Diagnostik und Therapie des Mammakarzinoms. Zuckschwerdt Verlag, München, Bern, Wien, New York 1998

Prechtel K: Begriffsbestimmung des nicht-invasiven und invasiven Karzinoms der Mamma. In: v. Bohmert $\mathrm{H}$ (Hrsg): Brustkrebs und Brustkonstruktion. Thieme Verlag, Stuttgart , New York 1982, 1-5

Purushotham AD (2003): The controversy of breast cancer screening. Lancet $\underline{358}$ : 1340-1342

Ratanachaikanont $T$ (2005): Clinical breast examination and its relevance to diagnosis of palpable breast lesion. J Med Assoc Thai $\underline{88}$ : 505-507

Riede UN, Schaefer HE: Allgemeine und spezielle Pathologie, 3. Auflage, Thieme Verlag, Stuttgart, New York 1993

Riede UN, Schaefer HE: Allgemeine und spezielle Pathologie. 4.Auflage, Thieme Verlag, Stuttgart, New York 1999

Ries L, Harkins D, Krapcho M, Mariotto A, Miller B, Feuer E, Clegg L, Eisner M, Horner M, Howländer N, Hayat B, Edwards B: SEER Cancer Statistics Review 19752003 (http://seer.cancer.gov/csr/1975 2003/). Natl Canc Inst 2006

Rodenko GN, Harms SE, Puneda JM, Farell RS, Evans WP, Copit DS, Krakos PA, Flamig DP (1996): MR Imaging in the Management of Lobular Carcinoma of the breast: correlation with Pathology. AJR 167: 1415-1419

Rosen PP, Groshen S (1990): Factors influencing survival and prognosis in early breast carcinoma (T1NOM0-T1N1M0). Assessment of 664 patients with median follow-up of 19 years. Surg Clin North Am $\underline{70}$ : 937-962

Rosen PP, Oberman HA: Tumors of the mammary gland. Atlas of tumor pathology, $3^{\text {rd }}$ ser, Fasc. 7. AFIP, Washington D.C. 1993

Roth-Ganter G: Mammographie-Handbuch für die tägliche Praxis, Thieme Verlag, Stuttgart, New York 2002 
S3-Leitlinie (interdisziplinär) für die Diagnostik, Therapie und Nachsorge des Mammakarzinoms. Deutsche Krebsgesellschaft e.V. Zuckschwerdt Verlag, München, Bern, Wien New York 2008

Samir SM, Fayaz MS, Elbasmi A, Motawy MM, Abuzallouf S, George T, Abdelhady M, Bedair A (2011): Medullary carcinoma of the breast: ten year clinical experience of the kuwait cancer control centre. Gulf J Oncolog 10: 45-52

Sardanelli F, Giuseppetti GM, Panizza P, Bazzocchi M, Fausto A, Simonetti G, Lattanzio V, Del Maschio A; Italian Trial for Breast MR in Multifocal/Multicentric Cancer (2004): Sensitivity of MRI versus mammography for detecting foci of multifocal, multicentric breast cancer in Fatty and dense breasts using the wholebreast pathologic examination as a gold standard. AJR Am J Roentgenol 183: 11491157

Sardanelli F, Podo F, Santoro F, Manoukian S, Bergonzi S, Trecate G, Vergnaghi D, Federico M, Cortesi L, Corcione S, Morassut S, Di Maggio C, Cilotti A, Martincich L, Calabrese M, Zuiani C, Preda L, Bonanni B, Carbonaro LA, Contegiacomo A, Panizza P, Di Cesare E, Savarese A, Crecco M, Turchetti D, Tonutti M, Belli P, Maschio AD; High Breast Cancer Risk Italian 1 (HIBCRIT-1) Study (2011): Multicenter surveillance of women at high genetic breast cancer risk using mammography, ultrasonography, and contrast-enhanced magnetic resonance imaging (the high breast cancer risk italian 1 study): final results. Radiology $\underline{46}$ : 94105

Schnall MD, Blume J, Bluemke DA, DeAngelis GA, DeBruhl N, Harms S, HeywangKöbrunner SH, Hylton N, Kuhl CK, Pisano ED, Causer P, Schnitt SJ, Thickman D, Stelling CB, Weatherall PT, Lehmann C, Gatsonis CA (2006): Diagnostic architectural and dynamic features at breast MR imaging: multicenter study. Radiology 238: 42-53

Schneider C, Fehr MK, Steiner RA, Hagen D, Haller U, Fink D (2003): Frequency and distribution pattern of distant metastases in breast cancer patients at the time of primary presentation. Arch Gynecol Obstet 269: 9-12

Schoppmann SF, Bayer G, Aumayr K et al. (2004): Prognostic value of lymphangiogenesis and lymphvascular invasion in invasive breast cancer. Ann Surg 240: 306-312

Schulz-Wendtland RE, Wenkel U, Aichinger M, Tartsch I, Kuchar, A, Bodicker C, Evertsz H.O, Peitgen W, Bautz (2003): [Film-screen mammography versus digital storage plate mammography: hard copy and monitor display of microcalcifications and focal findings--a retrospective clinical and histologic analysis]. Röfo Fortschr Geb Röntgenstr Neuen Bildgeb Verf 175: 1220-1224 
Sheppard DG, Whitman GJ, Huynh PT, Sahin AA, Fornage BD, Stelling CB (2000): Tubular carcinoma of the breast: mammographic and sonographic features. AJR Am J Roentgenol 174: 253-257

Shou J, Massarweh S, Osborne CK, Wakeling AE, Ali S, Weiss H, Schiff R (2004): Mechanisms of tamoxifen resistance: increased estrogen receptor-HER2/neu crosstalk in ER/HER2-positive breast cancer. J Natl Cancer Inst 96: 926-935

Sickles EA, Filly RA, Callen PW (1984): Benign breast lesions: ultrasound detection and diagnosis. Radiology 151: 467-470

Silverstein MJ (2003): The Universitiy of Southern California /Van Nuys prognostic Index for ductal carcinoma in situ of the breast. Am J Surg 186: 337-343

Silverstein MJ, Buchanan C (2003): Ductal carcinoma in situ: USC/Van Nuys Prognostic Index and the impact of margin status. Breast 12: 457-471

Silverstein MJ, Poller DN, Waisman JR, Colburn WJ, Barth A, Gierson ED, Lewinsky B, Gamagami P, Slamon DJ (1995): Prognostic classification of breast ductal carcinoma in-situ. Lancet $\underline{345}$ : 1154-1157

Singletary SE, Allred C, Ashley P, Bassett LW, Berry D, Bland KI, Borgen PI, Clark G, Edge SB, Hayes DF, Hughes LL, Hutter RV, Morrow M, Page DL, Recht A, Theriault RL, Thor A, Weaver DL, Wieand HS, Greene FL (2002): Revision of the American Join Committee on Cancer Staging system for breast cancer. J Clin Oncol 20: $3628-3636$

Sloane JP, Ellman R, Anderson TJ, Brown CL, Coyne J, Dallimore NS, Davies JD, Eakins D, Ellis IO, Elston CW et al. (1994): Consistency of histopathological reporting of breast lesions detected by screening: findings of the U.K. National External Quality Assessment (EQA) Scheme. UK National Coordinating Group for Breast Screening Pathology. Eur J Cancer 30A: 1414-1419

Sohn C, Tercanli S, Holzgreve W: Ultraschall in Gynäkologie und Geburtshilfe. 2. Auflage, Thieme Verlag, Stuttgart, New York 2003

Soo MS, Baker JA, Rosen EL, Vo TT (2002): Sonographically guided biopsy of suspicious microcalcifications of the breast: a pilot study. Am J Roentgenol 178: 1007-1015

Sutherland CM, Mather FJ (1986): Long-term survival and prognostic factors in breast cancer patients with localized (no skin, muscle, or chest wall attachment) disease with and without positive lymph nodes. Cancer 57: 622-629

Teifke A, Lehr HA, Vomweg TW, Hlawatsch A, Thelen M (2003): Outcome analysis and rational management of enhancing lesions incidentally detected on contrastenhanced MRI of the breast. AJR 181: 655-662 
Trecate G, Vergnaghi D, Manoukian S, Bergonzi S, Scaperrotta G, Marchesini M, Ferranti C, Peissel B, Spatti G, Bohm S, Conti A, Costa C, Sporeni M, Podo F, Musumeci R (2006): MRI in the early detection of breast cancer in women with high genetic risk. Tumori 92: 517-523

Trop I, Lalonde L, Mayrand MH, David J, Larouche N, Provencher D (2010): Multimodality breast cancer screening in women with a familial or genetic predisposition. Curr Oncol 17: 28-36

Tumorregister München: (www.tumorregister-muenchen.de)

Tumorzentrum Hamburg E.V.:(http://www.tumorzentrumhh.de/Leitlinien/dcis.html)

Tumorzentrum München: (www. tumorregistermuenchen.de/facts/specificanalysis.html)

Van Goethem M, Schelfout K, Dijckmans L, Van Der Auwera JC, Weyler J, Verslegers I, Biltjes I, De Schepper A (2004): MR mammography in the pre-operative staging of breast cancer in patients with dense breast tissue: comparison with mammography and ultasound. Eur Radiol 14: 809-817

Virnig BA, Shamliyan T, Tuttle TM, Kane RL, Wilt TJ (2009): Diagnosis and management of ductal carcinoma in situ (DCIS). Evid Rep Technol Assess 185: 1549

Vollenweider-Zerargui L, Barrelet L, Wong Y, Lemarchand-Beraud T, Gomez F (1986): The predictive value of estrogen and progesteron receptors concentrations on the clinical behavior of breast cancer in women. Cancer 57: 1171-1180

Weinstein SP, Orel SG, Heller R, Reynolds C, Czerniecki B, Solin LJ, Schnall M (2001): MR imaging of the breast in patients with invasive lobular carcinoma. AJR Am J Roentgenol 176: 399-406

Weismann C, Hergan K (2007): Aktueller Stand der 3D/4D-Volumensonografie der Mamma. Ultraschall Med 28: 273-282

Westerhof JP, Fischer U, Moritz JD, Oestermann JW, Grabbe E (1996): Accuracy of MR Imaging of the Breast in Patients with suspicious microcalcifications on Mammograms. Radiology 201: 129

Winnekendonk G, Krug B, Warm M, Gohring UJ, Mallmann P, Lackner K (2004): Diagnostischer Stellenwert der präoperativen MR-Mammographie. Röfo Fortschr Geb Röntgenstr Neuen Bildgeb Verfahr 176: 688-693

Wiratkapun C, Bunyapaiboonsri W, Wibulpolprasert B, Lertsithichai P (2010): Biopsy rate and positive predictive value for breast cancer in BI-RADS category 4 breast lesions. J Med Assoc Thai 93: 830-837 
Wulf $\mathrm{KH}$, Schmidt-Matthiesen H: Spezielle gynäkologische Onkologie II. Urban \& Schwarzenberg, München, Wien, Baltimore 1996

Wurdinger S, Kamprath S, Eschrich D, Schneider A, Kaiser WA (2001): False negative findings of malignant breast lesions on preoperative magnetic resonance mammography. Breast 10: 131-139

Yang WT, Tse GM (2004): Sonographic, mammographic, and histopathologic correlation of symptomatic ductal carcinoma in situ. AJR Am J Roentgenol 182: 101110

Yilmaz E, Lebe B, Balci P, Sal S, Canda T (2002): Comparison of mammographic and sonographic findings in typical and atypical medullary carcinomas of the breast. Clin Radiol ㅍ: 640-645

Young JL, JR, Ries LG, Pollack ES (1984): Cancer patient survival among ethnic groups in the United States. J Natl Cancer Inst $\underline{73}$ : 341-352

Zonderland HM, Coerkamp EG, Hermans J, van de Vijver MJ, van Voorthuisen AE (1999): Diagnosis of breast cancer: contribution of US as an adjunct to mammography. Radiology 213: 413-422 


\section{Anhang}

\subsection{Abbildungsverzeichnis}

\begin{tabular}{|ll|}
\hline Abb.4.1a: & Seite 36 \\
\hline Abb.4.1b: & Seite 36 \\
\hline Abb.4.2a: & Seite 37 \\
\hline Abb.4.2b: & Seite 38 \\
\hline Abb.4.2c: & Seite 38 \\
\hline Abb.4.2d: & Seite 39 \\
\hline Abb.4.2e: & Seite 39 \\
\hline Abb.4.2f: & Seite 40 \\
\hline Abb.4.2g: & Seite 40 \\
\hline Abb.4.2h: & Seite 41 \\
\hline Abb.4.2i: & Seite 41 \\
\hline Abb.4.2j: & Seite 42 \\
\hline Abb.4.3a: & Seite 43 \\
\hline Abb.4.3b: & Seite 44 \\
\hline Abb.4.3c: & Seite 44 \\
\hline Abb.4.3d: & Seite 45 \\
\hline Abb.4.3e: & Seite 45 \\
\hline Abb.4.4a: & Seite 47 \\
\hline Abb.4.4b: & 49 \\
\hline Abb.4.4d: & \\
\hline Abb.4.4e: & Seite \\
\hline Abb.4.4f: & 39 \\
\hline & \\
\hline
\end{tabular}




\begin{tabular}{|lc|}
\hline Abb.4.4g: & Seite 50 \\
\hline Abb.4.5.1a: & Seite 51 \\
\hline Abb.4.6.1a: & Seite 52 \\
\hline Abb.4.6.1b: & Seite 53 \\
\hline Abb.4.6.1c: & Seite 53 \\
\hline Abb.4.6.1d: & Seite 54 \\
\hline Abb.4.6.1e: & Seite 54 \\
\hline Abb.4.6.1f: & Seite 55 \\
\hline Abb.4.6.1g: & Seite 56 \\
\hline Abb.4.6.2a: & Seite 57 \\
\hline Abb.4.6.2.3a: & Seite 59 \\
\hline Abb.4.6.2.3b: & Seite 60 \\
\hline
\end{tabular}


8.2 Tabellenverzeichnis

\begin{tabular}{|lc|}
\hline Tab.1.3.1a: & Seite 10 \\
\hline Tab.1.4.1a: & Seite 17 \\
\hline Tab.3.1a: & Seite 30 \\
\hline Tab.3.1b: & Seite 30 \\
\hline Tab.3.1c: & Seite 31 \\
\hline Tab.3.1d: & Seite 31 \\
\hline Tab.3.1e: & Seite 32 \\
\hline Tab.3.1f: & Seite 33 \\
\hline Tab.5.3a: & Seite 65 \\
\hline Tab.5.3b: & Seite 65 \\
\hline
\end{tabular}




\subsection{Abkürzungsverzeichnis}

ACR: American College of Radiology

AC: adenoid-zystisches Karzinom

ADH: atypische duktale Hyperplasie

AGO: Arbeitsgemeinschaft für gynäkologische Onkologie

ATAC-Studie: Arimidex-Tamoxifen-Alone-or-in-Combination-Studie

BET: brusterhaltende Therapie

bFGF: basic Fibroblast Growth Factor

BIG-1-98-Studie: Breast International Group-1-98

BI-RADS: Breast imaging report and data system

BMI: Body Mass Index

BRCA 1: Breast Cancer Gene 1

BRCA 2: Breast Cancer Gene 2

bzw.: beziehungsweise

cc: kranio-kaudal

CC: muzinöses Karzinom

CDI: Color Doppler Imaging

CMF: Cyclophosphamid, Methotrexat, 5-Fluorouracil

CT: Computer Tomographie

CTLM: Computed-Tomography-Laser-Mammographie

CUP: Carcinoma of unknown primary

DCIS: Duktales Carcinoma in situ

DS: intraduktales Karzinom

EBCTCG: Early Breast Cancer Trialists`Collaborative Group 
EIC: extensive intraduktale Komponente

ER: Östrogenrezeptor

FISH: Fluoreszenz in-situ-Hybridisierung

FZM: fokal zystische Mastopathie

G: Grading

GE: Gradienten-Echo

ggf: gegebenenfalls

GKV: gesetzliche Krankenkassenversicherung

GnRH-Analoga: Gonadotropin Releasing Hormone

Gy: Gray

HER2/neu: Human Epidermal Growth Factor Receptor 2

HF: homogen lipomatös

HP: homogen parenchymal

HPF: high power field

I: inhomogen

ID, IDC: invasiv duktales Karzinom

IL, ILC: invasiv lobuläres Karzinom

IN: inflammatorisches Karzinom

IP:invasiv papilläres Karzinom

KM: Kontrastmittel

LCIS: Lobuläres Carcinoma in situ

LIN: lobuläre intraepitheliale Neoplasie

LM: latero-medial

MC: medulläres Karzinom 


\begin{tabular}{|c|}
\hline MCC: Multizentrizität \\
\hline ME: Mastektomie \\
\hline MFC: Multifokalität \\
\hline MG: Mammographie \\
\hline Mhz: Megahertz \\
\hline Mip: maximum intensitiy projection \\
\hline ML: medio-lateral \\
\hline MLO: medio-lateraler Strahlengang \\
\hline mm: Millimeter \\
\hline MRE: Magnetresonanzelastographie \\
\hline MRM: Magnetresonanztomographie der Mamma \\
\hline MRT: Magnetresonanztomographie \\
\hline MTRA: medizinisch-technische Radiologieassistenten/innen \\
\hline MX: Mammographie \\
\hline NHSBSP: National Coordinating Group for Breast Screening Pathology \\
\hline PAl-1: Plasminogen-Aktivator-Inhibitor-1-Protein \\
\hline PD: Morbus Paget der Mamille \\
\hline PGMI: (p) perfekt, (g) gut, (m) moderat, (i) inadäquat \\
\hline PR: Progesteronrezeptor \\
\hline ROI: region of interest \\
\hline SNB: Sentinel Node Biopsy \\
\hline sog: sogenannte \\
\hline TC: tubuläres Karzinom \\
\hline TNM: Tumorgröße (T), Lymphknotenbefall (N), Fernmetastasierung (M) \\
\hline
\end{tabular}


u.a: unter anderem

uPA: Plasminogenaktivator vom Urokinasetyp

US: Ultraschall

VEGF: Vascular Endothelial Growth Factor

VNPI: Van-Nuys-Prognose-Index

z.B.: zum Beispiel 


\section{Danksagung}

Mein besonderer Dank gilt Herrn Prof. Dr. med. U. Fischer für die Überlassung des Themas und die exzellente Betreuung dieser Dissertation.

Ganz herzlich bedanke ich mich bei den Mitarbeitern und Mitarbeiterinnen des Diagnostischen Brustzentrums in Göttingen für die große Unterstützung und Hilfe in Fachfragen. 
